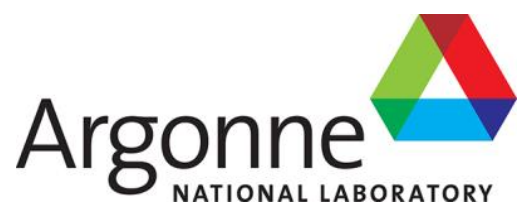

\title{
PROTEUS-SN User Manual
}

Revision 2.0

Nuclear Engineering Division 


\begin{abstract}
About Argonne National Laboratory
Argonne is a U.S. Department of Energy laboratory managed by UChicago Argonne, LLC under contract DE-AC02-06CH11357. The Laboratory's main facility is outside Chicago, at 9700 South Cass Avenue, Argonne, Illinois 60439. For information about Argonne and its pioneering science and technology programs, see www.anl.gov.
\end{abstract}

\title{
DOCUMENT AVAILABILITY
}

Online Access: U.S. Department of Energy (DOE) reports produced after 1991 and a growing number of pre-1991 documents are available free via DOE's SciTech Connect (http://www.osti.gov/scitech/)

\author{
Reports not in digital format may be purchased by the public from the \\ National Technical Information Service (NTIS): \\ U.S. Department of Commerce \\ National Technical Information Service \\ 5301 Shawnee Rd \\ Alexandra, VA 22312 \\ www.ntis.gov \\ Phone: (800) 553-NTIS (6847) or (703) 605-6000 \\ Fax: (703) 605-6900 \\ Email: orders@ntis.gov \\ Reports not in digital format are available to DOE and DOE contractors from the \\ Office of Scientific and Technical Information (OSTI): \\ U.S. Department of Energy \\ Office of Scientific and Technical Information \\ P.O. Box 62 \\ Oak Ridge, TN 37831-0062 \\ www.osti.gov \\ Phone: (865) 576-8401 \\ Fax: (865) 576-5728
}

\begin{abstract}
Disclaimer
This report was prepared as an account of work sponsored by an agency of the United States Government. Neither the United States Government nor any agency thereof, nor UChicago Argonne, LLC, nor any of their employees or officers, makes any warranty, express or implied, or assumes any legal liability or responsibility for the accuracy, completeness, or usefulness of any information, apparatus, product, or process disclosed, or represents that its use would not infringe privately owned rights. Reference herein to any specific commercial product, process, or service by trade name, trademark, manufacturer, or otherwise, does not necessarily constitute or imply its endorsement, recommendation, or favoring by the United States Government or any agency thereof. The views and opinions of document authors expressed herein do not necessarily state or reflect those of the United States Government or any agency thereof, Argonne National Laboratory, or UChicago Argonne, LLC.
\end{abstract}


ANL/NE-14/6 (Rev 2.0)

\section{PROTEUS-SN User Manual}

Revision 2.0

prepared by

Emily R. Shemon, Micheal A. Smith and Changho Lee

Nuclear Engineering Division, Argonne National Laboratory

July 31,2015 



\section{ABSTRACT}

PROTEUS-SN is a three-dimensional, highly scalable, high-fidelity neutron transport code developed at Argonne National Laboratory. The code is applicable to all spectrum reactor transport calculations, particularly those in which a high degree of fidelity is needed either to represent spatial detail or to resolve solution gradients. PROTEUS-SN solves the second order formulation of the transport equation using the continuous Galerkin finite element method in space, the discrete ordinates approximation in angle, and the multigroup approximation in energy. PROTEUS-SN's parallel methodology permits the efficient decomposition of the problem by both space and angle, permitting large problems to run efficiently on hundreds of thousands of cores. PROTEUS-SN can also be used in serial or on smaller compute clusters (10's to 100 's of cores) for smaller homogenized problems, although it is generally more computationally expensive than traditional homogenized methodology codes. PROTEUS-SN has been used to model partially homogenized systems, where regions of interest are represented explicitly and other regions are homogenized to reduce the problem size and required computational resources.

PROTEUS-SN solves forward and adjoint eigenvalue problems and permits both neutron upscattering and downscattering. An adiabatic kinetics option has recently been included for performing simple time-dependent calculations in addition to standard steady state calculations. PROTEUS-SN handles void and reflective boundary conditions. Multigroup cross sections can be generated externally using the $\mathrm{MC}^{2}-3$ fast reactor multigroup cross section generation code or internally using the cross section application programming interface (API) which can treat the subgroup or resonance table libraries.

PROTEUS-SN is written in Fortran 90 and also includes $\mathrm{C}$ preprocessor definitions. The code links against the PETSc, METIS, HDF5, and MPICH libraries. It optionally links against the MOAB library and is a part of the SHARP multi-physics suite for coupled multi-physics analysis of nuclear reactors.

This user manual describes how to set up a neutron transport simulation with the PROTEUS-SN code. A companion methodology manual describes the theory and algorithms within PROTEUS-SN. 


\section{TABLE OF CONTENTS}

Abstract

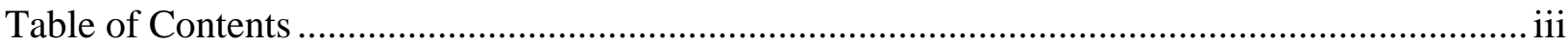

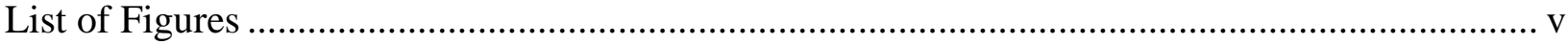

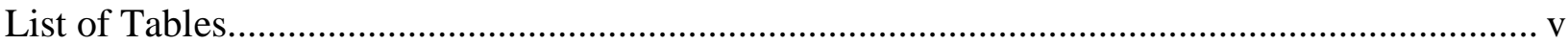

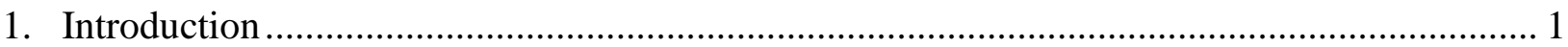

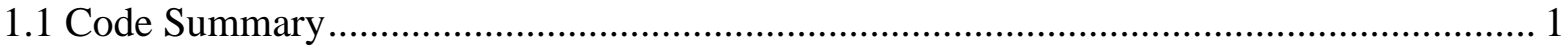

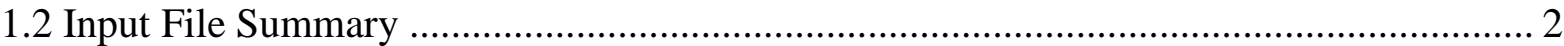

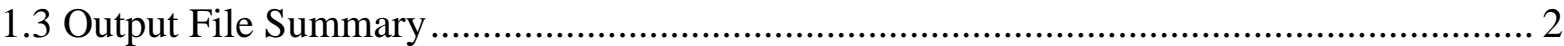

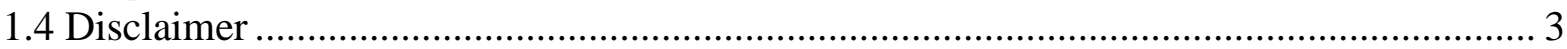

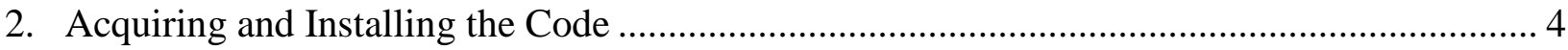

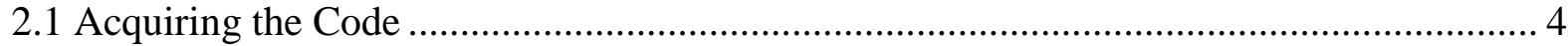

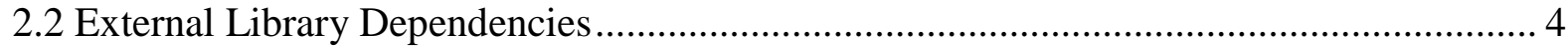

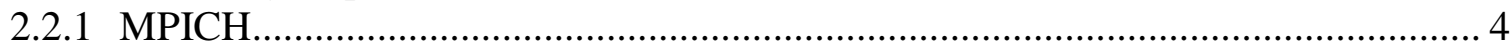

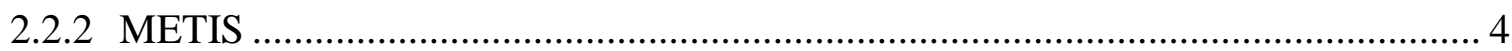

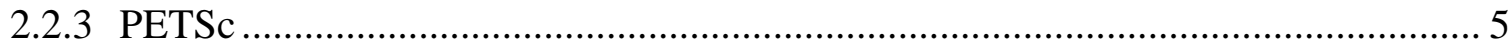

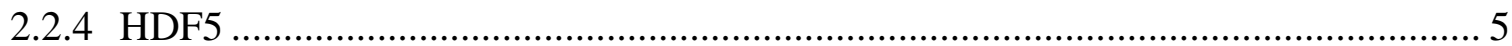

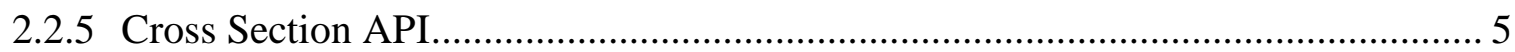

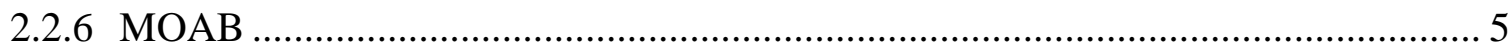

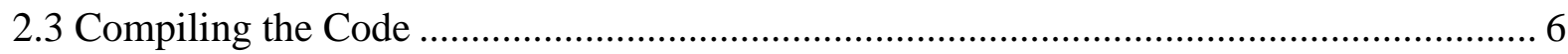

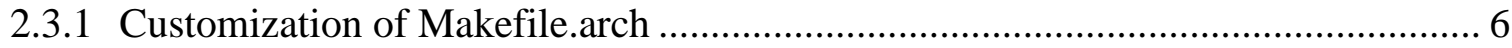

2.3.2 Customization of PROTEUS_Preprocess.h ………............................................ 7

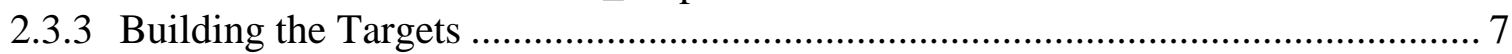

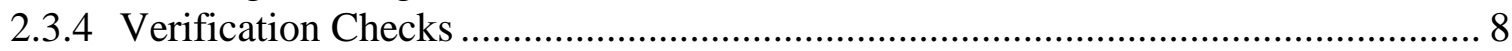

2.3.5 Recommended Compilers and Architectures ………………………………..... 8

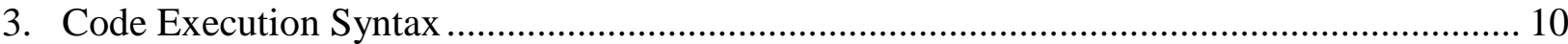

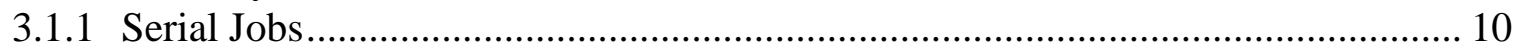

3.1.2 Parallel Jobs ............................................................................................. 10

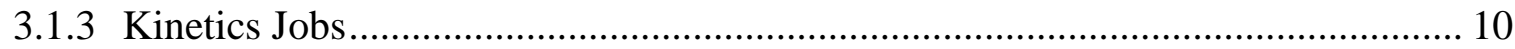

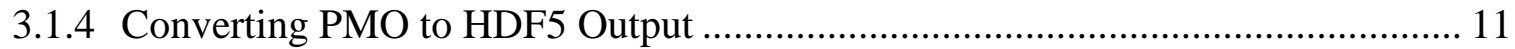

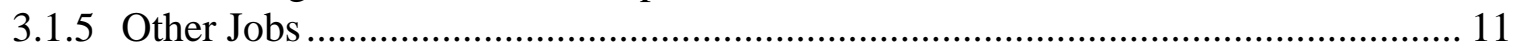

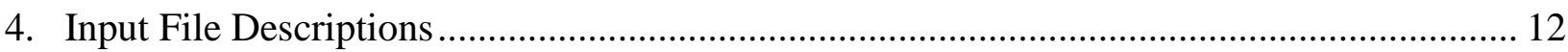

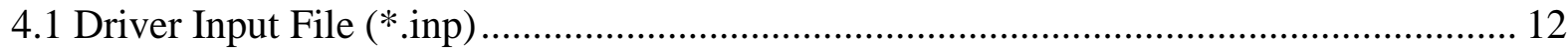

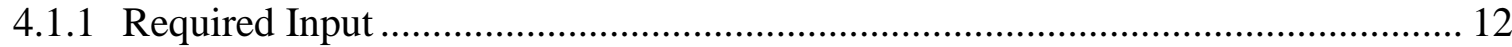

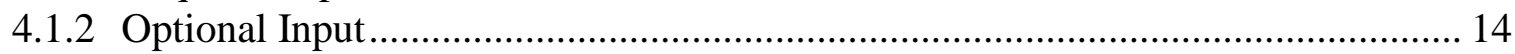

4.1.3 Assigning Boundary Conditions....................................................................... 19

4.1.4 Parallel Partitioning Control .......................................................................... 20

4.1.5 Processing Cross Sections with the Cross Section API........................................ 21

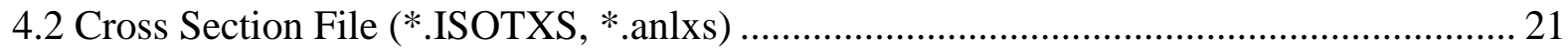

4.3 Mesh File $(*$.nemesh, *.ascii, *.pntmesh, *.bgpmesh) .................................................. 22

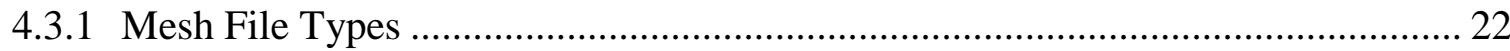

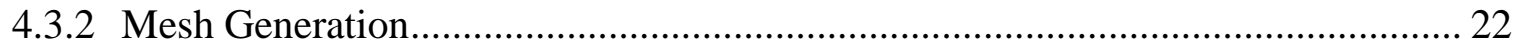

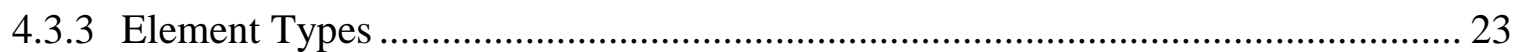

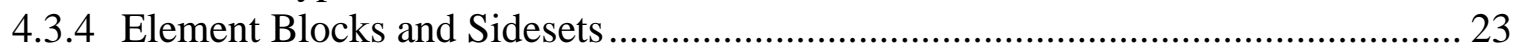


4.3.5 Issues to Consider While Meshing .......................................................................... 24

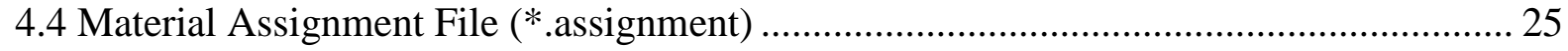

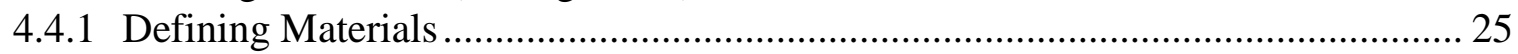

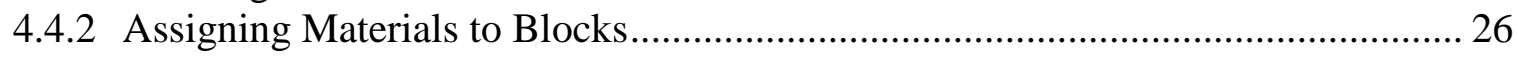

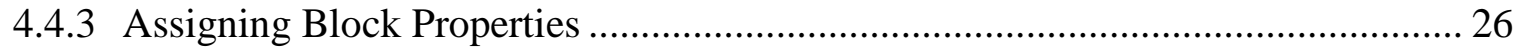

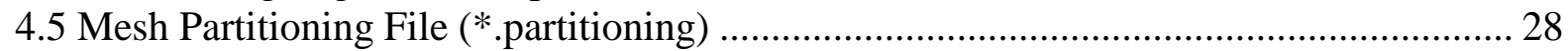

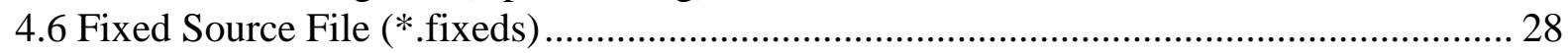

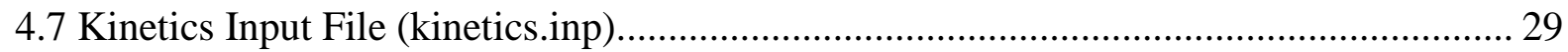

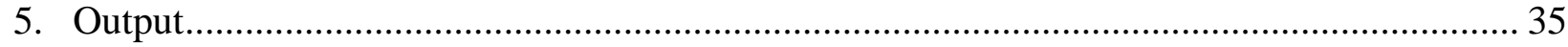

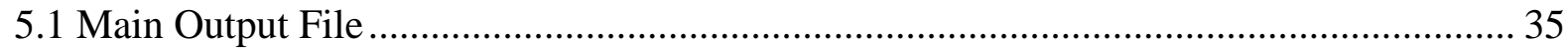

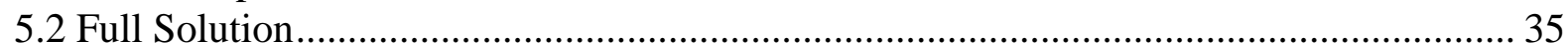

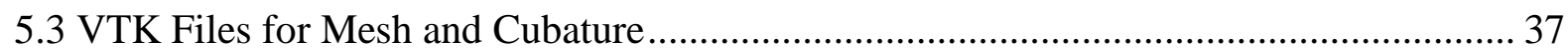

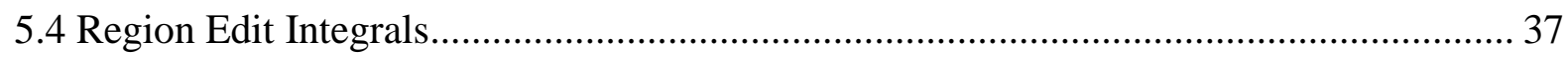

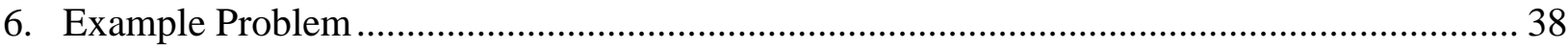

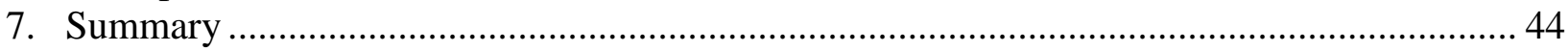

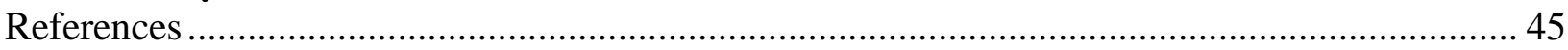

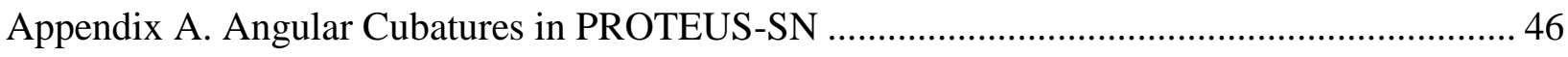

Appendix B. Example PROTEUS-SN Driver Input File: “sn2nd.inp" ................................... 54

Appendix C. Cross Section File Format for PROTEUS-SN: "anlxs” "..................................... 55

Appendix D. Mesh File Format Specification for PROTEUS-SN: "nemesh"............................ 58

Appendix E. Mesh File Format Specification for PROTEUS-SN: “ascii” ............................... 63

Appendix F. Example PROTEUS-SN Text Output File........................................................ 66

Appendix G. Finite Elements Used in PROTEUS-SN Along with Vertex and Surface

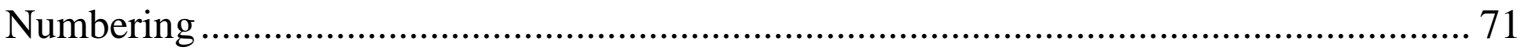




\section{LIST OF FIGURES}

Figure 1. Recommended Customizations in Makefile.arch.................................................... 6

Figure 2. Assigning Boundary Conditions at Runtime in Driver Input File........................... 19

Figure 3. Example Mesh Showing Blocks and Sidesets for 1/8-th Pin Cell............................ 23

Figure 4. Non-conformal Mesh (left) and Conformal Mesh (right). ...................................... 24

Figure 5. Material Definition in the Assignment File (Atom Fractions). ................................. 25

Figure 6. Material Definition in the Assignment File (Weight Fractions)............................... 25

Figure 7. Recursive Material Definition in the Assignment File. ............................................ 26

Figure 8. Assigning a Material to a Block in the Assignment File......................................... 26

Figure 9. Assigning Total Atom Density to a Block in the Assignment File. ........................ 27

Figure 10. Assigning Total Mass Density to a Block in the Assignment File. ........................ 27

Figure 11. Example PROTEUS-SN Assignment File............................................................... 27

Figure 12. Example Kinetics Input File with No Fixed Source............................................... 33

Figure 13. Example Kinetics Input File with Fixed Source.................................................... 34

Figure 14. Visualization of Geometry and Mesh Used to Analyze the Advanced Test Reactor.

Figure 15. ATR fast (left) and Thermal (right) Flux Calculated by PROTEUS-SN. ......... 36

Figure 16. Visualization of 2D Fast (Left) and Thermal (Right) Fluxes for ATR Calculation.

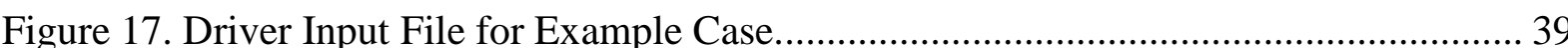

Figure 18. Cross Section File for Example Case. .............................................................. 40

Figure 19. Mesh File for Example Case. ……………………........................................... 41

Figure 20. VTK Mesh (left) and VTK Mesh with Block Coloring (right) for Example Case. 42

Figure 21. Material Assignment File for Example Case........................................................... 42

Figure 22. Nu*fission Rate in Test Case Plotted in VisIt. ...................................................... 43

\section{LIST OF TABLES}

Table 1. Possible Customizations in PROTEUS_Preprocess.h .................................................. 7

Table 2. List of PROTEUS-SN Makefile Targets (Executables). .............................................. 7

Table 3. Required Input for Driver Input File ....................................................................... 12

Table 4. Auxiliary Input Controls in Driver Input File …………….................................... 14

Table 5. Fixed Source File Format....................................................................................... 29

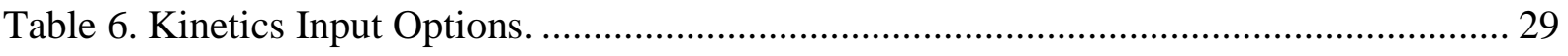





\section{Introduction}

PROTEUS-SN is a highly scalable, discrete ordinates-based 3D neutron transport code targeted for high-fidelity nuclear reactor applications. This document provides a comprehensive user guide for PROTEUS-SN. The purpose of this user guide is to familiarize new users with the basics of carrying out simulations using the PROTEUS-SN code.

\subsection{Code Summary}

PROTEUS-SN is a three-dimensional deterministic neutron transport code which solves the second order formulation of the neutron transport equation. The second order "even-parity" form of the transport equation is discretized using the continuous Galerkin finite element method in space, the discrete ordinates approximation in angle, and the multigroup approximation in energy. A finite element mesh, discrete ordinates cubature, and multigroup cross section data set must therefore be provided.

PROTEUS-SN solves both forward and adjoint eigenvalue problems with Tchebychev acceleration and a fixed Wielandt shift. Fixed source problems are also treated and the code handles neutron upscattering and downscattering. An adiabatic kinetics option has recently been included in the code for performing simple time-dependent calculations in addition to standard steady state calculations. PROTEUS-SN handles void and reflective boundary conditions. PROTEUS-SN does not perform gamma radiation transport but accounts for gamma power production assuming that the gammas are absorbed at the location where they are emitted.

The overall solution technique in PROTEUS-SN is discretization of the even-parity transport equation into a linear system of equations for the even-parity component of the angular flux. The within-group system of equations is solved using the SSOR-preconditioned conjugate gradient method in the PETSc library, where scattering iterations are performed using Richardson iteration with diffusion synthetic acceleration. The multigroup system of equations is solved using Gauss-Seidel iteration. PROTEUS-SN permits parallelization of neutron transport problems in both space and angle which significantly reduces in the per-processor memory load for a given problem. The efficient parallel techniques in PROTEUS-SN permit the solution of very large problems. PROTEUS has been demonstrated to solve 500 billion - 1 trillion degrees of freedom on leadership computing resources.

PROTEUS-SN is written in Fortran 90 source code and also includes $\mathrm{C}$ preprocessor definitions. The code links against various open source libraries including a parallel linear algebra solver (PETSc), a mesh decomposition library (METIS) and a portable output library (HDF5). 


\subsection{Input File Summary}

Four input files are required to perform PROTEUS calculations (three other files are relevant only for certain simulations):

a. Driver input file

b. Multigroup cross section file or cross section library file

c. Mesh file

d. Material assignment file

e. (Parallel execution mode only and optional) Mesh partitioning file

f. (Kinetics only) Kinetics input file

g. (Fixed source only) Fixed source input file

The driver input file is a keyword-based free format text file that contains the simulation parameters such as angular cubature, parallelization, convergence criteria, and iteration limits.

The multigroup cross section file is a formatted file containing the multigroup cross sections for all the isotopes or materials of interest. Multigroup cross sections are provided in either *.anlxs or *.ISOTXS formatted files. The latter is the native file format for Argonne's $\mathrm{MC}^{2}-3$ fast reactor multigroup cross section generation code [1] and is also the compatible format for Argonne's well-established neutronics code, DIF3D [2].

The mesh file is a formatted file containing information about the finite element mesh. PROTEUS-SN handles unstructured meshes with the triangular (2D), quadrilateral (2D), triangular prism (3D), tetrahedral (3D) or hexahedral (3D) elements. A combination of elements of the same dimensionality can be used in the same mesh provided they are in different blocks. The basis functions are polynomial, and linear or quadratic order is typically used.

The material assignment file is a keyword-based free format text file that specifies the material assignment to regions in the mesh, as well as defining properties such as density.

The mesh partitioning file is relevant only for parallel simulations. It is automatically generated during a simulation and can be re-used in future simulations using the same mesh and mesh decomposition.

The kinetics input file is relevant only for kinetics simulations. It describes the timesteps to be taken, including material assignments for each timestep, and the delay cross section data and fixed source data to be used, if applicable.

The fixed source input file is relevant only for fixed source simulations. It can be used in either steady state or kinetics mode.

\subsection{Output File Summary}

PROTEUS-SN produces a main text output file containing an echo of the inputs, eigenvalue iteration history, and MPI timing summaries. Optionally, the full solution is exported to HDF5 
file format and can be visualized using VisIt [3] with the "UNIC" plugin. Additionally, blockintegrated quantities (power, fission, absorption, and group flux) can be exported. Finally, the cubature and mesh can also be individually exported to a VTK [4] format.

\subsection{Disclaimer}

As of the time of this document's release, PROTEUS-SN has not had sufficient testing and development to be considered as a production level tool. The reliability of PROTEUS is therefore not guaranteed. Additionally, the accuracy of PROTEUS has not been assessed on a wide range of reactor physics problems. In many cases, performance and efficiency have been sacrificed in favor of providing a working capability which prevents one from making a realistic computational effort comparison to other available tools. In many cases, reliable algorithms have been chosen to compensate for memory shortages on high performance computing machines which degrade the wall clock performance of the existing code. 


\section{Acquiring and Installing the Code}

This chapter briefly describes how to obtain and install PROTEUS-SN. It lists the various external library dependencies and also recommended compilers. For more specific (and up-todate) compilation information including configuration options of the various packages, one should consult the installation documentation included with the distribution.

\subsection{Acquiring the Code}

The PROTEUS-SN source code is export-controlled and currently obtained through Argonne National Laboratory. Contact nera-software@anl.gov for distribution information. The distribution package includes source code, build instructions, benchmark examples and documentation.

PROTEUS-SN is dependent on various open source libraries which are freely available and therefore not included in the distribution. Specific versions of the libraries may be required to ensure compatibility with PROTEUS-SN, given that the various packages change function interfaces periodically. These external package dependencies as well as compiler recommendations are described in the next section.

\subsection{External Library Dependencies}

PROTEUS-SN achieves distributed memory parallelization using the Message Passing Interface (MPI) protocol and uses the MPI2 standard typically through the MPICH2 [5] library implementation. PROTEUS-SN also has essential dependencies on two external packages, the mesh partitioning package METIS [6] and the parallel linear algebra solver PETSc [8]. Additionally, PROTEUS-SN optionally interfaces with data format library HDF5 [9] and the mesh frameworks tool MOAB [10]. We briefly discuss these packages here. For help installing any of the packages for use with PROTEUS-SN, refer to the documentation included with the distribution, or contact nera-software@anl.gov.

\subsubsection{MPICH}

PROTEUS-SN supports the MPI2 standard with most of the testing work done with MPICH2 version 1.4 or later. The MPICH library provides the ability to perform parallel communication between processors in distributed memory mode. Various versions of MPICH2 are available at http://www.mcs.anl.gov/research/projects/mpich2staging/goodell/downloads/tarballs/. Refer to the installation documentation in the distribution for guidance on which version to use.

\subsubsection{METIS}

PROTEUS-SN uses the METIS package [6,7] to determine the mesh partitioning scheme for spatial domain decomposition. To download METIS, visit the webpage http://glaros.dtc.umn.edu/gkhome/fsroot/sw/metis/OLD. PROTEUS-SN is currently configured to use METIS 4.0. Later versions of METIS incorporate different function interfaces which are not guaranteed to work with the PROTEUS-SN implementation. 


\subsubsection{PETSC}

The PETSc [8] package is used by PROTEUS-SN to solve an assembled parallel matrixvector preconditioning system, and at this time PROTEUS-SN is configured to run with PETSc version 3.1or PETSc version 3.4 due to major changes in the PETSc interfaces in later versions. Work is being done to update PROTEUS-SN to work with later versions of PETSc. We note that the current PROTEUS distribution assumes the user is linking against PETSc 3.1. To instead link to PETSC 3.4, the user must configure PROTEUS at compile time by defining the PROTEUS_Use_PETSC3_4 pre-processing variable in PROTEUS_Preprocess.h.

While advanced users can set up more complicated preconditioners, PROTEUS-SN by default uses the Symmetric Successive Over-Relaxation (SSOR) preconditioned Conjugate Gradient (CG) solver of PETSc. The primary benefit of this solver is its well-demonstrated parallel scalability and ease of use. The primary weaknesses include exceedingly high memory requirements and failure to efficiently utilize the full power of the platform which it is deployed upon (5\% of peak performance). While we have researched ways into overcoming these limitations by replacing this preconditioner, time constraints have prevented us from making alternate preconditioner options available at this time.

\subsubsection{HDF5}

The HDF5 [9] software is used by PROTEUS-SN to store the flux solution for follow-up visualization purposes. Recent versions (1.10.0+) of VisIt [12] include a plugin called "UNIC" which can be used to directly read this data (files with .h5 extensions). The HDF5 software can be problematic for most novice users to install, and we strongly advise consulting nerasoftware@anl.gov if difficulties arise. PROTEUS-SN has successfully run with HDF5-1.8.10 and later versions should also work provided the function interfaces do not change.

\subsubsection{Cross Section API}

The Cross Section API is an external library developed by Argonne National Laboratory which processes multigroup cross sections using the subgroup or resonance table method. In order to use the Cross Section API, a transport code should provide a one-group fixed source transport solver to it. The Cross Section API can be requested with PROTEUS-SN starting in June 2014. PROTEUS-SN includes an interface to the Cross Section API such that multigroup cross sections can be calculated on-the-fly for the exact heterogeneous geometry of interest rather than providing pre-processed multigroup data. This implementation is new and considered a "beta" version, so use it with caution.

\subsubsection{MOAB}

The MOAB [10] package can be used by PROTEUS-SN to load meshes directly from a variety of mesh generator formats and perform dynamics coupling. Unfortunately, the interface to MOAB is not stable and could not be released at this time. 


\subsection{Compiling the Code}

Detailed compilation instructions are provided with the distribution, including suggested configuration options for many of the external dependencies. Once the external library dependencies have been downloaded and built, PROTEUS-SN is compiled using the provided makefile in the /source directory of the distribution. The makefile includes a second file, Makefile.arch, also located in the same directory.

\subsubsection{Customization of Makefile.arch}

Makefile.arch is a text file in the /source directory which contains typical compiler options and flags. We recommend you modify Makefile.arch to add an architecture-specific compilation section for your machine. Figure 1 shows an excerpt from Makefile.arch for a hypothetical machine called "MyMachineName".



Figure 1. Recommended Customizations in Makefile.arch.

We recommend that you add a section to Makefile.arch for your machine similar to above where the machine name should be recovered earlier in the script using the "uname" or "dnsdomainname" UNIX functions. In the section shown above, you should assign the MPICH compiler locations, PETSc installation root location, PETSc architecture (such that the PETSC_DIR variable is the full install path), and METIS, HDF5, MOAB, and Cross Section API 
installation locations. Note that the F77 compiler must be specified individually as it is used to compile one of the kinetics routines.

\subsubsection{Customization of PROTEUS_Preprocess.h}

The header file PROTEUS_Preprocess. $h$ shown in Table 1 controls various data assignments via pre-processor directives. Most of the contents of this file should remain unchanged for routine use. However, the top few definitions can be commented or uncommented to enable debug printing, and to enable compilation with optional dependencies (MOAB, HDF5, and Cross Section API).

Table 1. Possible Customizations in PROTEUS_Preprocess.h

\begin{tabular}{|l|l|}
\hline Preprocessing Directive Variable & Meaning (if defined) \\
\hline PROTEUS_Debug & Enable debug printing \\
\hline PROTEUS_CallTree & Enable call tree printing \\
\hline PROTEUS_InterfacesWithMOAB & $\begin{array}{l}\text { Enable MOAB (reading .h5m mesh, multiphysics) } \\
\text { (keep disabled unless you have the MOAB version) }\end{array}$ \\
\hline PROTEUS_InterfacesWithHDF5 & Enable HDF5 for output (output .h5 type) \\
\hline PROTEUS_InterfacesWithSGAPI & Enable Cross Section API \\
\hline PROTEUS_Use_PETSC3_4 & Link against PETSc 3.4 instead of PETSc 3.1 \\
\hline PROTEUS_SQS_Precursors_By_Block & Enable storage by block (keep disabled) \\
\hline
\end{tabular}

Note that the HDF5 and Cross Section library locations must also be specified in Makefile.arch if they are uncommented in PROTEUS_Preprocess.h. Since the MOAB interface is not stable for release at this time, the \#define PROTEUS_InterfacesWithMOAB line should be commented out.

\subsubsection{Building the Targets}

Once you have customized Makefile.arch and PROTEUS_Preprocess.h, invoke the "make all" command from Linux command prompt inside the /source directory. This command creates all of the targets (executables) in Table 2. Alternatively, type "make <target>" at the command line to create only a specific target.

Table 2. List of PROTEUS-SN Makefile Targets (Executables).

\begin{tabular}{|l|l|}
\hline Target & Application \\
\hline sn2nd.x & Steady state version of PROTEUS-SN \\
\hline sn2nd_kinetics.x & Kinetics version of PROTEUS-SN \\
\hline
\end{tabular}




\begin{tabular}{|l|l|}
\hline sn2nd_sg.x & $\begin{array}{l}\text { Steady state version of PROTEUS-SN using Cross Section API } \\
\text { for cross section generation }\end{array}$ \\
\hline regression_gauss.x & Testing purposes (regression tests) \\
\hline regression_libs.x & Testing purposes (regression tests) \\
\hline PMO_AssembleHDF5.x & Converts set of .pmo files to a single .h5 file \\
\hline
\end{tabular}

We note that parallel make execution is possible on many platforms using "make -j4 $<$ target>".

\subsubsection{Verification Checks}

Both unit tests and benchmark problems are available to check whether installation was successful. The benchmark problems are provided in the /benchmark directory. The directory "SN2ND" contains 7 steady state benchmark problems. The directory "SN2ND_Kinetics" contains 4 kinetics benchmark problems. Reference solutions are provided for a variety of parallel decompositions (typically using up to 12 processors).

To perform the full suite of verification checks including unit tests and benchmark problems, use the command "make verify". To run only the steady state benchmark problems, use the command "make bench". To run only the kinetics benchmark problems, use the command "make bench_kinetics". The benchmark problems will be performed by a script and compared to the reference solutions.

\subsubsection{Recommended Compilers and Architectures}

PROTEUS-SN has been regularly compiled with the Intel 10.1.15 compiler on Intel hardware. We do not suggest using Intel compiler versions 10.1.20 through all of version 11 (except 11.1.080) as they were unreliable with the various dependencies and PROTEUS-SN source code. Intel versions 12.1+ are required for use with the Cross Section API as it contains FORTRAN 2003 constructs and requires a FORTRAN 2003-compliant compiler.

PROTEUS-SN has also been compiled with the latest IBM XL Fortran compilers for the BlueGene P and Q systems (Intrepid and Mira at ALCF). Additionally, PROTEUS-SN has been compiled on a Cray XT5 system (Jaguar at ORNL). The code should work on most Linux workstations, clusters, and laptops as well as Macs.

The current version of the code does not utilize shared memory parallelism and thus does not utilize threading or GPUs.

Compilation with GNU compilers should be relatively straightforward using the flags "-ffreeline-length-0 -ffixed-line-length-0" on the FFLAGS line in Makefile.arch. However, the code is 
PROTEUS-SN User Manual

July 31, 2015

not routinely tested with the GNU compilers so minor changes may be required for successful compilation. 


\section{Code Execution Syntax}

\subsubsection{Serial Jobs}

PROTEUS-SN is executed via command line on Linux platforms. To perform a serial job with the steady state solver, type the following at the command prompt (assuming the executable is in the current directory or in the user's path):

\$> sn2nd.x -input/path/to/mydriver.inp

The -input flag argument specifies that PROTEUS-SN should look for a specific driver input file in the location specified by the following argument. If the "-input" flag and argument is omitted, PROTEUS-SN looks for the driver input file in the current working directory with the default name "sn2nd.inp". The output can be redirected from standard output to a file using the -output flag.

\subsubsection{Parallel Jobs}

To execute the steady state solver in parallel mode, type the following:

\$> mpiexec -n 8 sn2nd.x -input/path/to/mydriver.inp

The mpiexec command is standard with MPICH and specifies that 8 processors should be used in this example. The parallel decomposition of the problem is fully specified by the number of processors and the value of SEGMENT_ANGLE in the driver input. The problem is first partitioned by angle into SEGMENT_ANGLE processors. The case of SEGMENT_ANGLE=0 is a special case discussed in Section 4.1.4. For SEGMENT_ANGLE $>0$, the remaining factor of processors (in this case, 8/SEGMENT_ANGLE) is automatically dedicated to spatial decomposition. The spatial mesh is decomposed at runtime based on these numbers. Parallelism is discussed further in the next chapter.

\subsubsection{Kinetics Jobs}

The kinetics solver is run identically to the above except using a different executable:

\$> mpiexec -n 4 sn2nd_kinetics.x -input/path/to/mydriver.inp

As in the steady state solver case, only the driver input file name is specified. The kinetics input file is also required and assumed to reside in the working directory and have the default name "kinetics.inp". To specify an alternative kinetics input file name, use the following syntax:

\$> mpiexec -n 4 sn2nd_kinetics.x -input /path/to/mydriver.inp kinetics MyKinetics.inp 


\subsubsection{Converting PMO to HDF5 Output}

The PMO_AssembleHDF5.x executable converts a set of .pmo files to a single HDF5 file. It runs in serial and requires three arguments:

\$> PMO_AssembleHDF5.x mymesh.nemesh color.inp myfluxfile.h5

The first argument to PMO_AssembleHDF5.x is the mesh file (.nemesh, .ascii, .pntmesh, or .bgpmesh) which was used in the problem. The second input is an optional color map. The color map is a formatted text file containing on each line the 16-character region name (REGION_xxxxxxxxx), followed by a space and then the color number (an integer). Executing the program with no arguments will list the color number options. If you do not wish to provide a color map, keep the "color.inp" argument but remove any files with the same name in the directory to avoid runtime errors about incorrect file formatting.

The last argument is the name of the desired HDF5 output file. It must have the same prefix as the prefix of the PMO files since the executable will automatically search the directory for PMO files with the same prefix. For example, to convert several files named "MyOutput0000001.pmo", "MyOutput0000002.pmo", etc. to an HDF5 file, the last argument in the command should be "MyOutput.h5".

\subsubsection{Other Jobs}

Job execution on leadership computing platforms and other queued platforms should follow the syntax required by the relevant job submission system. 


\section{Input File Descriptions}

This section describes the required input files for a PROTEUS-SN calculation:

- Driver input file

- Cross section file

- Mesh file

- Material assignment file

Additionally, three other input files are used for parallel runs, kinetics jobs, and fixed source problems:

- (Parallel mode, optional) Mesh partitioning file

- (Kinetics jobs only) Kinetics input file

- (Fixed source jobs only) Fixed source input file

An example driver input file is provided in Appendix B.

\subsection{Driver Input File (*.inp)}

Upon execution, PROTEUS looks for the driver input file, "sn2nd.inp", in the current working directory. This input file is a plain text (ASCII) file that drives the PROTEUS calculation by specifying solver tolerances, the angular discretization, parallelization options, and other input options. Additionally, the UNIX file paths to the other input files (cross sections, mesh, and material assignment file) are specified. Input options are specified in the file by special keywords which can appear in any order.

To use a different file name for the driver input such as "sn2nd_5g_mesh1.inp", add the command line option "-input sn2nd_5g_mesh1.inp" at execution to alert PROTEUS of the driver input file name.

\subsubsection{Required Input}

The following table shows the essential driver level input required in every PROTEUS-SN calculation. The keywords and values are not case sensitive. Default values are listed when applicable.

Table 3. Required Input for Driver Input File

\begin{tabular}{|c|l|c|l|}
\hline Keyword & \multicolumn{1}{|c|}{ Input Data } & Default Value & \multicolumn{1}{c|}{ Description } \\
\hline \multirow{5}{*}{ SN_TYPE } & 1D Options: & & \\
& LEGENDRE 1-D & & \\
& DOUBLE LEGENDRE & & Specifies the type of SN \\
& 2D/3D Options: & & cubature to use. \\
& CARLSON_EM & & \\
& CARLSON_LM & & \\
\hline
\end{tabular}




\begin{tabular}{|c|c|c|c|}
\hline & $\begin{array}{l}\text { CARLSON_EQUALW } \\
\text { LEG-TCHEBY } \\
\text { X_DIR_LEG-TCHEBY } \\
\text { DOUB_LEG-TCHEBY } \\
\text { TRI_LEG-TCHEBY } \\
\text { LEBEDEV-LAIKOV } \\
\text { TEG_CORNER_EQWT } \\
\text { TEG_CORNER_LSQ } \\
\text { TEG_CENTROID_EQWT } \\
\text { TEG_CENTROID_LSQ } \\
\text { THURGOOD_TN } \\
\text { THURGOOD_LEASTSQ } \\
\text { COBE_EVEN_EQWT } \\
\text { COBE_EVEN_LSQ } \\
\text { COBE_ODD_EQWT } \\
\text { COBE_ODD_LSQ } \\
\text { PYRAMID_CARLSON } \\
\text { COBE_C_EVEN_EQWT } \\
\text { COBE_C_EVEN_LSO } \\
\text { COBE_C_ODD_EQWT } \\
\text { COBE_C_ODD_LSQ }\end{array}$ & & \\
\hline THETA_RESOLUTION & $<$ Integer $>0>$ & - & $\begin{array}{l}\text { Specifies the polar angle } \\
\text { resolution of the SN } \\
\text { cubature. }\end{array}$ \\
\hline PHI_RESOLUTION & $<$ |nteger $>0>$ & - & $\begin{array}{l}\text { Only applicable for } \\
\text { product cubatures such } \\
\text { as Leg-Tcheby. } \\
\text { Specifies the azimuthal } \\
\text { angle }(x, y) \text { resolution of } \\
\text { the SN cubature. }\end{array}$ \\
\hline SOLVE_TYPE & $\begin{array}{l}\text { FORWARD } \\
\text { ADJOINT } \\
\text { BOTH }\end{array}$ & ВОTH & $\begin{array}{l}\text { Only applicable for } \\
\text { steady state jobs. } \\
\text { Specifies which } \\
\text { calculations to perform } \\
\text { (forward, adjoint, or } \\
\text { both). }\end{array}$ \\
\hline SOURCEFILE_MESH & <Max 128 Characters> & - & $\begin{array}{l}\text { Specifies the UNIX file } \\
\text { path to a spatial } \\
\text { geometry mesh file }\end{array}$ \\
\hline SOURCEFILE_XS & <Max 128 Characters> & - & $\begin{array}{l}\text { Specifies the Unix file } \\
\text { path to a cross section } \\
\text { data file }\end{array}$ \\
\hline SOURCEFILE_MATERIAL & <Max 128 Characters $>$ & - & Specifies the Unix file \\
\hline
\end{tabular}



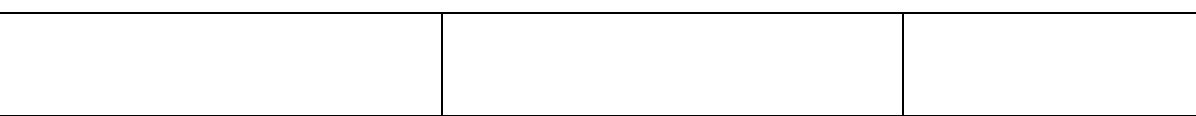

path to a material mapping file

The SN_TYPE input describes the type of cubature to be used in the calculation. Appendix A contains additional information about the usage of these cubatures, including spherical harmonics integration order and number of discrete directions (ordinates). The number of directions that will be used in PROTEUS-SN 3D calculations is given in the $2 \pi$ column of each table. From Table 3, there are two controls to select the resolution settings of a cubature, THETA_RESOLUTION and PHI_RESOLUTION, noting that the latter is only important for product cubatures (see Appendix A).

The SOLVE_TYPE input in Table 3 is only appropriate for steady state eigenvalue problems and allows the independent selection of the forward or adjoint flux solutions, or consecutive solves of both. In the case of both, the resulting flux file will only contain the forward flux solution. For kinetics and dynamics simulations, auxiliary drivers (executables) are provided which eliminate the need for this input option.

The remaining inputs in Table 3 specify the paths to the auxiliary input files (cross sections, mesh, and material assignments). Relative file paths are permitted and are assumed to be relative to the driver input file. For the mesh, an external file is always required using the SOURCEFILE_MESH input which has four native file formats: nemesh, ascii, pntmesh, and bgpmesh. These file formats are discussed later in this chapter. The SOURCEFILE_XS and SOURCEFILE_MATERIAL specify the cross section file and material assignment file, respectively.

\subsubsection{Optional Input}

Other optional driver-level input options are listed in Table 4. They can be classified into the following groups: debugging options, parallelization options, iterative solver options, cross section options, boundary condition options, multi-physics coupling options, and output file options. Brief explanations of each option as well as the default values are provided.

Table 4. Auxiliary Input Controls in Driver Input File

\begin{tabular}{|c|c|c|c|}
\hline Keyword & Input Data & $\begin{array}{c}\text { Default } \\
\text { value }\end{array}$ & Description \\
\hline \hline \multicolumn{3}{|c|}{ Auxiliary Input File Options } \\
\hline SOURCEFILE_FIXEDSOURCE & $<$ Max 128 Characters> & - & $\begin{array}{l}\text { Specified path to fixed } \\
\text { source input file for a fixed } \\
\text { source problem }\end{array}$ \\
\hline \multicolumn{4}{|c|}{ Debugging Options } \\
\hline
\end{tabular}




\begin{tabular}{|c|c|c|c|}
\hline Keyword & Input Data & $\begin{array}{c}\text { Default } \\
\text { value } \\
\end{array}$ & Description \\
\hline SKIPSOLVE & $\begin{array}{l}\text { YES } \\
\text { NO }\end{array}$ & NO & $\begin{array}{l}\text { Specifies whether to set } \\
\text { up the problem only } \\
\text { (YES) or to set up the } \\
\text { problem and solve it } \\
\text { (NO). }\end{array}$ \\
\hline DEBUG_PRINT_LEVEL & $\begin{array}{c}<\text { Integer greater than } \\
\text { or equal to } 0>\end{array}$ & 0 & $\begin{array}{l}\text { Specify level of debug } \\
\text { printing for entire } \\
\text { routine. }\end{array}$ \\
\hline DEBUG_PRINT_SETUP & $\begin{array}{c}<\text { Integer greater than } \\
\text { or equal to } 0>\end{array}$ & 0 & $\begin{array}{l}\text { Specify level of debug } \\
\text { printing of setup related } \\
\text { operations. }\end{array}$ \\
\hline DEBUG_PRINT_FORMATION & $\begin{array}{c}<\text { Integer in range } \\
{[0 . .10]>}\end{array}$ & 0 & $\begin{array}{l}\text { Controls debug printing } \\
\text { during the } \\
\text { tracking/matrix } \\
\text { formation ( } \max 10) .\end{array}$ \\
\hline DEBUG_PRINT_OUTER & $\begin{array}{c}<\text { Integer in range } \\
{[0 . .10]>}\end{array}$ & 0 & $\begin{array}{l}\text { Controls the debug } \\
\text { printing during the outer } \\
\text { iterations ( } \max 10) \text {. }\end{array}$ \\
\hline \multicolumn{4}{|c|}{ Parallelization Options } \\
\hline SEGMENT_ANGLE & $\begin{array}{c}<\text { Integer greater than } \\
\text { or equal to } 0>\end{array}$ & 0 & $\begin{array}{l}\text { The number of segments } \\
\text { to attempt in the angular } \\
\text { approximation. } \\
0=\text { Code decides max } \\
\text { parallelization } \\
1=\text { Serial } \\
2 \text { and up = \# Processors } \\
\text { (segments) in angle }\end{array}$ \\
\hline SOURCEFILE_MESHPART & <Max 128 Characters $>$ & - & $\begin{array}{l}\text { For space-parallel } \\
\text { simulations, specifies the } \\
\text { UNIX file path to a pre- } \\
\text { computed partitioning } \\
\text { index file for the mesh. }\end{array}$ \\
\hline \multicolumn{4}{|c|}{ Iterative Solver Options } \\
\hline EIGENVALUE_GUESS & $<$ Real Value $>$ & 1.0 & $\begin{array}{l}\text { Guess for the initial } \\
\text { eigenvalue. }\end{array}$ \\
\hline USE_TCHEBYCHEV_ACCEL & $\begin{array}{l}\text { YES } \\
\text { NO }\end{array}$ & YES & $\begin{array}{l}\text { Controls Tchebychev } \\
\text { acceleration of the } \\
\text { fission source. }\end{array}$ \\
\hline ITERATIONS_FISSION & $\begin{array}{c}<\text { Integer greater than } \\
\text { or equal to } 0>\end{array}$ & 100 & $\begin{array}{l}\text { Maximum number of } \\
\text { outer (fission source) } \\
\text { iterations. }\end{array}$ \\
\hline
\end{tabular}




\begin{tabular}{|c|c|c|c|}
\hline Keyword & Input Data & $\begin{array}{l}\text { Default } \\
\text { value }\end{array}$ & Description \\
\hline TOLERANCE_EIGENVALUE & $<$ Real Value $>$ & $1.0 \mathrm{E}-6$ & $\begin{array}{l}\text { Targeted relative error } \\
\text { on the eigenvalue }\end{array}$ \\
\hline TOLERANCE_FISSION & $<$ Real Value $>$ & $5.0 \mathrm{E}-6$ & $\begin{array}{l}\text { Targeted relative error } \\
\text { on the fission source }\end{array}$ \\
\hline TOLERANCE_FLUX & $<$ Real Value $>$ & $1.0 \mathrm{E}-7$ & $\begin{array}{l}\text { Targeted relative error } \\
\text { on the flux solution }\end{array}$ \\
\hline ITERATIVE_IMPROVEMENT & $<$ Real Value $>$ & 0.1 & $\begin{array}{l}\text { Upper limit targeted } \\
\text { iterative improvement } \\
\text { within each call. Value } \\
\text { will be adjusted down as } \\
\text { needed by the code. }\end{array}$ \\
\hline ITERATIONS_MAXUPSCATTER & $\begin{array}{c}<\text { Integer greater than } \\
\text { or equal to } 0>\end{array}$ & 5 & $\begin{array}{l}\text { Maximum Gauss-Seidel } \\
\text { "upscatter" iterations per } \\
\text { outer (not a fixed } \\
\text { iteration scheme). }\end{array}$ \\
\hline ITERATIONS_MINUPSCATTER & $\begin{array}{c}<\text { Integer greater than } \\
\text { or equal to } 0>\end{array}$ & 5 & $\begin{array}{l}\text { Minimum Gauss-Seidel } \\
\text { "upscatter" iterations per } \\
\text { outer (not a fixed } \\
\text { iteration scheme). }\end{array}$ \\
\hline ITERATIONS_SCATTERING & $\begin{array}{c}<\text { Integer greater than } \\
\text { or equal to } 0>\end{array}$ & 3 & $\begin{array}{l}\text { Maximum number of } \\
\text { source iterations per } \\
\text { within group iteration } \\
\text { (not a fixed iteration } \\
\text { scheme). }\end{array}$ \\
\hline ITERATIONS_SA_CG & $\begin{array}{c}<\text { Integer greater than } \\
\text { or equal to } 0>\end{array}$ & 1000 & $\begin{array}{l}\text { Maximum number of } \\
\text { within-group CG } \\
\text { iterations to perform. }\end{array}$ \\
\hline ITERATIONS_PETSC & $\begin{array}{c}<\text { Integer greater than } \\
\text { or equal to } 0>\end{array}$ & 1000 & $\begin{array}{l}\text { Maximum number of CG } \\
\text { iterations to use in all } \\
\text { levels of the PETSC } \\
\text { preconditioner tree. }\end{array}$ \\
\hline USE_PRECONDITIONER & $\begin{array}{l}\text { SSOR } \\
\text { ICC }\end{array}$ & SSOR & $\begin{array}{l}\text { Select the preconditioner } \\
\text { in PETSC. SSOR and ICC } \\
\text { are the only two allowed } \\
\text { at this time. }\end{array}$ \\
\hline USE_DSA & $\begin{array}{l}\text { YES } \\
\text { NO }\end{array}$ & YES & $\begin{array}{l}\text { Applies synthetic } \\
\text { diffusion acceleration to } \\
\text { the source iteration. }\end{array}$ \\
\hline RELAX_SPATIAL & $\begin{array}{l}\text { YES } \\
\text { NO }\end{array}$ & YES & $\begin{array}{l}\text { Relaxes spatial matrix } \\
\text { integration (minimize } \\
\text { effort). }\end{array}$ \\
\hline
\end{tabular}




\begin{tabular}{|c|c|c|c|}
\hline Keyword & Input Data & $\begin{array}{c}\text { Default } \\
\text { value }\end{array}$ & Description \\
\hline USE_WEILANDT_SHIFT & $\begin{array}{c}<\text { Real value greater } \\
\text { than eigenvalue }>\end{array}$ & - & $\begin{array}{l}\text { Apply a Weilandt shift to } \\
\text { the system to accelerate } \\
\text { convergence. Value must } \\
\text { be greater than largest } \\
\text { eigenvalue in system to } \\
\text { maintain positive definite } \\
\text { system. }\end{array}$ \\
\hline BASIC_BWO & $\begin{array}{l}\text { YES } \\
\text { NO }\end{array}$ & NO & $\begin{array}{l}\text { Applies a basic } \\
\text { bandwidth optimization } \\
\text { to mesh (reorder mesh } \\
\text { for minimum bandwidth } \\
\text { with ICC preconditioner). } \\
\text { This option is seldom } \\
\text { used to due } \\
\text { recommended SSOR } \\
\text { preconditioner. }\end{array}$ \\
\hline \multicolumn{4}{|c|}{ Cross Section Options } \\
\hline USE_CSAPI, USE_XSAPI & $\begin{array}{c}\text { NO } \\
\text { SUBGROUP (or SG) } \\
\text { RESONANCETABLE } \\
\text { (or RT) }\end{array}$ & NO & $\begin{array}{l}\text { Indicates whether the } \\
\text { subgroup or resonance } \\
\text { table cross section library } \\
\text { is to be used to generate } \\
\text { cross sections. The cross } \\
\text { section libraries are } \\
\text { expected for } \\
\text { heterogeneous geometry } \\
\text { problems. }\end{array}$ \\
\hline USE_TRANSPORT_XS & $\begin{array}{l}\text { YES } \\
\text { NO }\end{array}$ & NO & $\begin{array}{l}\text { Indicates that the } \\
\text { transport corrected cross } \\
\text { section is to be used as } \\
\text { the "total" cross section. } \\
\text { All anisotropic scattering } \\
\text { data is ignored. }\end{array}$ \\
\hline SCATTERING_ORDER & $\begin{array}{c}<\text { Integer greater than } \\
\text { or equal to } 0>\end{array}$ & 0 & $\begin{array}{l}\text { Legendre expansion } \\
\text { order of the scattering } \\
\text { kernel. }\end{array}$ \\
\hline
\end{tabular}




\begin{tabular}{|c|c|c|c|}
\hline Keyword & Input Data & $\begin{array}{c}\text { Default } \\
\text { value }\end{array}$ & Description \\
\hline SCATTERING_CHANGETOTAL & $\begin{array}{l}\text { YES } \\
\text { NO }\end{array}$ & no & $\begin{array}{l}\text { For some cross section } \\
\text { sets, the total cross } \\
\text { section is expanded into } \\
\text { spherical harmonics. YES } \\
\text { will auto-correct the } \\
\text { within group scattering } \\
\text { components. }\end{array}$ \\
\hline SCATTERING_SETUPTYPE & $\begin{array}{l}0 \\
1 \\
2\end{array}$ & 0 & $\begin{array}{l}\text { Indicates whether the } \\
2 \mathrm{~L}+1 \text { factor is not } \\
\text { included }(0), \text { multiplied } \\
(1), \text { or divided (2). }\end{array}$ \\
\hline \multicolumn{4}{|c|}{ Boundary Condition Options } \\
\hline BC_ALIAS & side_set_<\# $><B C T Y P E>$ & & $\begin{array}{l}\text { Assigns boundary } \\
\text { condition at runtime to } \\
\text { side set \#. Valid BCTYPE } \\
\text { names are VOID or } \\
\text { REFLECTIVE. }\end{array}$ \\
\hline \multicolumn{4}{|c|}{ Multi-physics Coupling Options (SHARP version only) } \\
\hline SN2ND_COMPUTES_DENSITY & $\begin{array}{l}\text { YES } \\
\text { NO }\end{array}$ & NO & $\begin{array}{l}\text { For coupling simulations, } \\
\text { tells PROTEUS-SN to } \\
\text { compute the densities } \\
\text { based on temperature } \\
\text { feedback. }\end{array}$ \\
\hline \multicolumn{4}{|c|}{ Output File Options } \\
\hline MESHSIMULATE & $<$ Integer $\geq 0>$ & 0 & $\begin{array}{l}\text { If this is non-zero, then a } \\
\text { pntmesh will be exported } \\
\text { using this value as the } \\
\text { number of processors. }\end{array}$ \\
\hline EXPORT_CUBATURE & $\begin{array}{l}\text { YES } \\
\text { NO }\end{array}$ & NO & $\begin{array}{l}\text { Used to export the SN } \\
\text { cubature to VTK file for } \\
\text { visualization purposes. }\end{array}$ \\
\hline EXPORT_XS_PRINT & $\begin{array}{l}\text { YES } \\
\text { NO }\end{array}$ & NO & $\begin{array}{l}\text { Controls printing of XS } \\
\text { data on rank } 0 .\end{array}$ \\
\hline EXPORT_MESH & $\begin{array}{l}\text { YES } \\
\text { NO }\end{array}$ & NO & $\begin{array}{l}\text { Export the local NTmesh } \\
\text { as "mesh.ascii" } \\
\text { formatted file. }\end{array}$ \\
\hline EXPORT_MESH_VTK & $\begin{array}{l}\text { YES } \\
\text { NO }\end{array}$ & NO & $\begin{array}{l}\text { Export the local NTmesh } \\
\text { to a VTK file for } \\
\text { visualization. }\end{array}$ \\
\hline
\end{tabular}




\begin{tabular}{|l|c|c|l|}
\hline \multicolumn{1}{|c|}{ Keyword } & Input Data & $\begin{array}{c}\text { Default } \\
\text { value }\end{array}$ & \multicolumn{1}{c|}{ Description } \\
\hline \hline EXPORT_FLUX & YES & NO & $\begin{array}{l}\text { Indicates whether full } \\
\text { solution (scalar flux, } \\
\text { reaction rates) are to be } \\
\text { exported to file. }\end{array}$ \\
\hline EXPORT_FILE & LMax 128 Characters \\
ending in .pmo or .h5 $>$ & default.h5 & $\begin{array}{l}\text { Specifies the flux file } \\
\text { name designation of a } \\
\text {.h5 (requires HDF5) or } \\
\text {.pmo file to export the } \\
\text { solution. }\end{array}$ \\
\hline EXPORT_EDITS & <Max 128 Characters $>$ & (none) & $\begin{array}{l}\text { Specifies that you would } \\
\text { like to export the region } \\
\text { edit integrals to an } \\
\text { output file (i.e. region } \\
\text { number, volume, flux, } \\
\text { etc) }\end{array}$ \\
\hline THERMAL_POWER & <Real & 1.0 & $\begin{array}{l}\text { Specifies the power } \\
\text { normalization in Watts } \\
\text { for the output file. }\end{array}$ \\
\hline
\end{tabular}

\subsubsection{Assigning Boundary Conditions}

The keyword BC_ALIAS in the driver input file is used to assign boundary conditions at runtime to the sidesets in the mesh. When converting an Exodus file to a PROTEUS-SN mesh format, the converted mesh will not contain the necessary boundary conditions. The boundary conditions should therefore be assigned using this input option. Figure 2 shows an example of how to assign boundary conditions to sidesets in the driver input file.

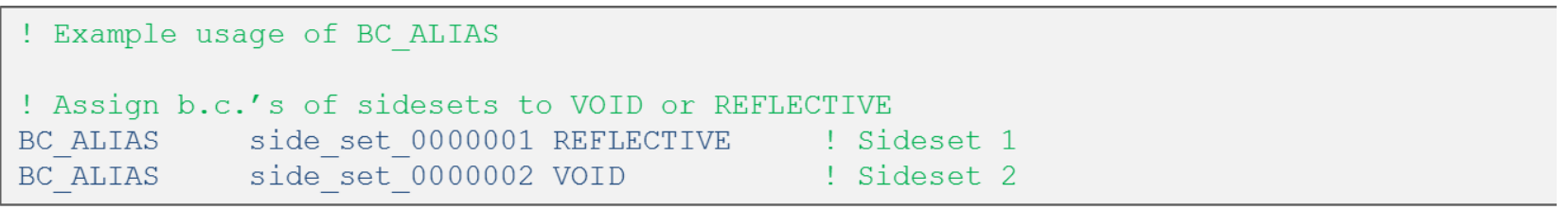

Figure 2. Assigning Boundary Conditions at Runtime in Driver Input File.

Note the convention for referring to sidesets in PROTEUS-SN. If the sideset number in the mesh is 1 , then the corresponding name in the driver input file should be "side_set_", then that number prepended by 0's to create 7 digits: side_set_0000001.

Possible boundary conditions are "VOID" or REFLECTIVE". If your .ascii or .nemesh file format already contains the boundary condition assignment, this option can be used to override it. 


\subsubsection{Parallel Partitioning Control}

PROTEUS-SN can partition the degrees of freedom with respect to both angle and space. It performs this partitioning on-the-fly after determining the number of available processors at execution time.

PROTEUS-SN always attempts to partition in angle first using the keyword SEGMENT_ANGLE to select the number of processors into which the angular work (number of angles) is divided. The number of angles simulated in PROTEUS-SN is only half the angles in the cubature due to symmetry of the even-parity angular flux. If unsure of the number of angles in the cubature, you can run the problem in serial with "SKIPSOLVE YES", which will echo the number of angles in the output.

Thus, SEGMENT_ANGLE can be any positive integer which is a common factor of the total number of processors and the number of angles used to discretize the angular space. If this rule is not satisfied, PROTEUS aborts with a message stating that some of the processes were not used. The default setting (0) tells PROTEUS-SN to maximize the parallelization in angle, such that PROTEUS-SN assigns the minimum number of angles to each process, given the constraint that each process has the same number of angles. Thus the only rule in that case is that the number of processes used is a multiple of the number of angles.

Once the number of angle partitions has been determined, PROTEUS-SN computes the number of spatial processors per angle partition such that it uses the full number of processes. In this context, the mesh is decomposed into the same exact partition for all angle groups. PROTEUS-SN typically performs the spatial decomposition of the mesh at runtime; however, this step can be memory intense. An input partitioning file can be provided using SOURCEFILE_MESHPART to skip this runtime expense. See Section 4.5 regarding mesh partitioning for more details.

For best scaling performance, each processor's angular partition should be assigned at least 2 angles. The optimal value of SEGMENT_ANGLE therefore depends on both the number of available processors and the number of angles in the cubature. Additionally, for best performance, each processer's spatial partition should have at least 1500 vertices. This number has been shown experimentally to provide a good balance between work and communication. These two partition sizes suggest a soft upper limit on how many processors should be used to solve a given problem (max processors $=$ \#vertices $/ 1500 *$ \#angles/2).

Let us imagine that we have 16 processors available to solve a problem with 8 angles and 10,000 vertices. Possible decompositions include 1x16 (8 angles and 625 vertices per process), $2 \times 8$ ( 4 angles and 1,250 vertices per process) or 4x4 ( 2 angles and 2,500 vertices per process), or $8 \times 2$ ( 1 angles and 5,000 vertices per process). We note that the best performance will probably be obtained at the $4 \times 4$ decomposition since it uses 2 angles per process and more than 1,500 vertices per process. However, the other decompositions are also valid. Additionally, fewer than 16 processors could be used to solve the problem. 
We note that the number of locally owned vertices (vertices per process) will likely vary from processor to processor in a real problem, whereas the number of locally owned angles will be constant across processors.

\subsubsection{Processing Cross Sections with the Cross Section API}

As noted earlier, PROTEUS-SN can be compiled with the cross section API (developed by Argonne National Laboratory) to process multi-group cross section data on-the-fly for the exact geometry using the subgroup or resonance table method. This capability is still being verified fully vetted and we recommend using with caution.

To process cross sections with the Cross Section API rather than providing an input cross section file, the line "USE_CSAPI YES" should appear in the driver input file. This option is only valid with the sn2nd_sg.x (PROTEUS-SN with cross section dependency) executable. The isotope names in the material assignment file must match those in the cross section library. If you are interested in using this option, please contact nera-software@ anl.gov for more up-to-date information on reliability.

\subsection{Cross Section File (*.ISOTXS, *.anlxs)}

The cross section file consists of the multigroup cross sections for all isotopes and/or compositions in the problem. Multigroup cross sections must be provided in one of the following formats:
a. *.ISOTXS (binary file)
b. *.anlxs (ASCII file)

The ISOTXS format is the preferred file format for cross section data used by PROTEUS-SN. The $\mathrm{MC}^{2}-3$ code (Argonne National Laboratory) can be used to process multigroup cross sections in this format. Additionally, the DRAGON code (Ecole Polytechnique de Montreal) [11] has a capability to generate ISOTXS file.

The anlxs file format is a simple ASCII interpretation of the data provided in ISOTXS. Any anlxs file format that is provided is converted into the appropriate ISOTXS file at runtime. A detailed description and an example of the anlxs file format are given in Appendix C.

The cross section file does not need to be located in the working directory. The UNIX file path to the cross section file must always be specified in the driver input file using the SOURCEFILE_XS keyword.

As an alternative to providing pre-processed multigroup cross sections data, PROTEUS-SN can process cross section data on-the-fly using the exact heterogeneous geometry via the Cross Section Application Programming Interface (API) (see 4.1.5). 


\subsection{Mesh File (*.nemesh, *.ascii, *.pntmesh, *.bgpmesh, *.ufmesh)}

\subsubsection{Mesh File Types}

PROTEUS-SN is an unstructured finite-element based method and requires a finite element mesh as input. One of the following finite element mesh formats is expected:
a. *.nemesh
b. *ascii
c. *.pntmesh (parallel execution on Linux)
d. *.bgpmesh (parallel execution for special lower memory machines)
e. *.ufmesh

The nemesh file format is a simple finite element mesh input file format which is detailed in Appendix D. The ascii file format is less user friendly, and relies upon structures intrinsic to the PROTEUS-SN code but is detailed in Appendix E.

The pntmesh file format is the base file that PROTEUS-SN will run with when executing (it converts the nemesh or ascii files into pntmesh formats at runtime). The bgpmesh file format is a pre-decomposed ascii file format for use with the memory-limited BlueGene/Q supercomputer at Argonne.

The ufmesh file format is a user-friendly mesh format which uses keywords to describe the geometry and mesh for a regular 2D Cartesian or hexagonal assembly or pin cell. Different ufmesh files can be merged together to create 3D cores quickly and conveniently using PROTEUS's mesh utility tools. A companion manual [13] is available describing the use of ufmesh and associated mesh utility tools. Note that ufmesh permits the naming of regions by character string rather than by number for a more intuitive input file and analysis procedure.

\subsubsection{Mesh Generation}

PROTEUS-SN uses the mesh file formats described above. There is no finite element meshing software that directly produces these types of files, so it is recommended to use your favorite meshing software and build your own converter to the nemesh or ascii formats. Note that the GAMBIT neutral file format is an ASCII format comparable (but not identical) to the nemesh file format.

We routinely use the CUBIT software [14] to build an EXODUS II (.e or .exo) mesh. The PROTEUS package includes a separate EXODUS II converter to transfer EXODUS II meshes into the ascii file format required by PROTEUS. Note that this converter is built on NetCDF [15] which is downloaded separately and is not part of the distribution. The EXODUS II converter is compatible with CUBIT versions 12 and 13, and may also work with CUBIT 14 (not tested).

Alternatively, the open source tool MeshKit [16] can be used to create $h 5 m$ type meshes, which can be converted to Exodus format via MOAB's conversion tool [17]. 
As a final note, we have some internal tools to help with mesh creation, for example, merging a collection of assemblies into a core, or extruding a 2D mesh into 3D. These are currently not being released with PROTEUS-SN, but if interested please contact nera-software@anl.gov.

\subsubsection{Element Types}

PROTEUS-SN permits bar (1D), triangular (2D), quadrilateral (2D), tetrahedral (3D), prism (3D), and hexahedral (3D) element shapes. Additionally, PROTEUS accepts elements with linear, quadratic, and higher orders. For fine mesh geometries (fuel pin-cells), it is strongly advised to utilize linear finite elements in PROTEUS-SN because of the severe condition number problems associated with the even-parity method. For coarse homogeneous problems, quadratic order finite elements are typically best. Note that PROTEUS-SN can handle a mixture of element types in any given mesh (i.e. combination of triangular and quadrilateral) provided each block contains elements of a single type.

More information on the PROTEUS-SN finite element zoo is available in Appendix G. Note that the reference surface numbering in the nemesh file format refers to the element-wise reference surface numbering in Appendix G.

\subsubsection{Element Blocks and Sidesets}

An element block is a user-defined set of elements (not necessarily contiguous) which have the same element type and same material composition. A sideset is a user-defined set of surfaces (not necessarily contiguous) which have the same boundary conditions (see Figure 3).

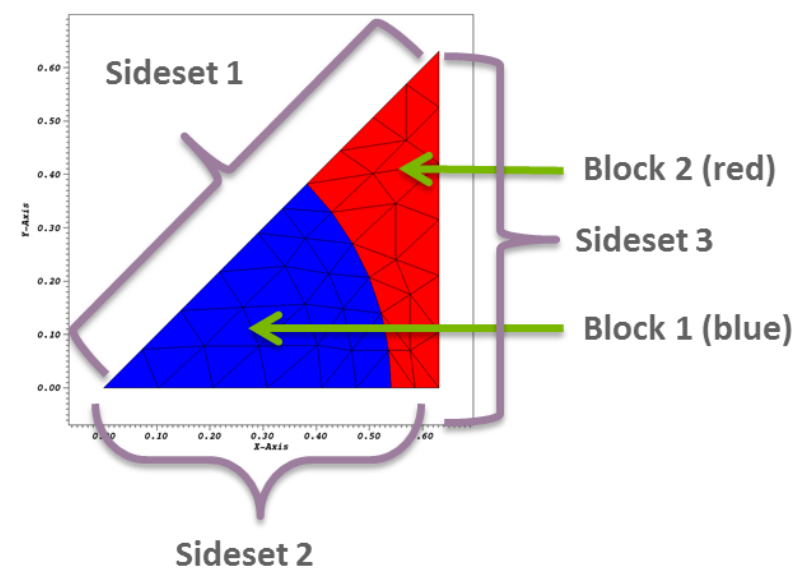

Figure 3. Example Mesh Showing Blocks and Sidesets for 1/8-th Pin Cell.

In most mesh generators, one assigns names and block numbers to physical volumes in the domain. For example, a block can be created out of a set of elements which all have "fuel" composition and are linear triangles. The elements in those volumes are typically written together in the mesh file. However, the names are rarely passed through to the interface file, 
which is the case for CUBIT-created Exodus files, despite the fact that names would be more convenient for most neutronics work. Instead, only the block numbers are passed through.

In PROTEUS-SN, the preferred approach is to utilize names and thus the block numbers are translated into names. Focusing on the nemesh file format of Appendix B as an example, each element is assigned a "region" number. This region number, say "1", is translated to the name "REGION_000000001" in the mesh conversion process. This block name is then used in the assignment file to assign cross section data (defined via compositions) to regions of the domain. Similarly, sidesets of similar surfaces can be defined in the Exodus mesh and assigned boundary conditions as described in Section 4.1.3. Extraneous (unused) sidesets should not be defined in the mesh as they can cause issues.

\subsubsection{Issues to Consider While Meshing}

PROTEUS-SN requires a conformal mesh, i.e. a mesh where a node of one element is also a node of the neighboring element (unless it lies on the boundary). Figure 4 shows an example of each type.
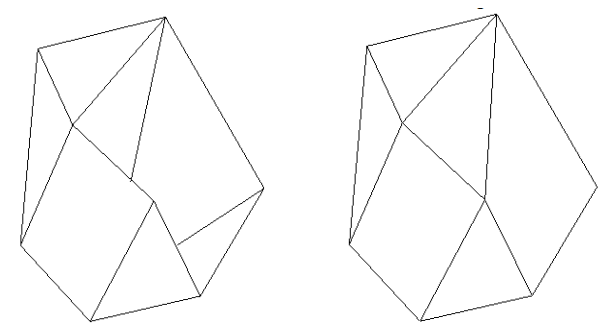

Figure 4. Non-conformal Mesh (left) and Conformal Mesh (right).

In addition to being conformal, element quality is an important consideration in the mesh. Long, thin elements can cause trouble. The ideal elements in 2D are an equilateral triangle or square.

As noted above, for fine mesh geometries (fuel pin-cells), it is strongly advised to utilize linear finite elements in PROTEUS-SN because of the severe condition number problems associated with the even-parity method. For coarse homogeneous problems, quadratic order finite elements are typically best.

Finally, note that linear elements do not exactly preserve curvilinear geometry. Therefore, in a mesh refinement study with linear elements and curvilinear geometry, one should be careful to preserve the important masses (fuel, absorber) manually by tweaking the densities. The CUBIT program has convenient functions to query the volume of the elements and blocks.

Note that the computational effort required in PROTEUS-SN increases roughly linearly with increasing number of vertices (maintaining the dimensionality). Going from 2D to 3D results in a significant increase in computational effort due to the increased number of elements and increased connectivity. 


\subsection{Material Assignment File (*.assignment)}

The material assignment (*.assignment) file performs three main functions: (1) define materials or mixtures based on the isotopes in the cross section file, (2) assign these materials to blocks in the mesh, and (3) assign properties (e.g. density) to blocks in the mesh.

The material assignment file uses simple keyword-based input in free format. It must be created by hand by the user, although scripting procedures can often be developed to speed the process. Comments start with either "!" or "\#”.

\subsubsection{Defining Materials}

The user-defined isotope or composition names in the anlxs or ISOTXS files are the base materials we can work with. These base materials can be directly assigned to blocks. Additionally, new materials can be defined as mixtures of the base materials.

Figure 5 demonstrates the definition of a material called FUEL from 8 compositions appearing in the cross section file, "U234A", "U235A", etc. The keyword MATERIAL_DEF is used recursively to add compositions to the material FUEL with the given atom fractions. The sum of all atom fractions is renormalized to 1 inside PROTEUS-SN. In this way, the true atom densities can be given (in \#/barn-cm) for easier recordkeeping.

\begin{tabular}{|lll|}
\hline MATERIAL_DEF & FUEL U234A & $2.7572 \mathrm{E}-5$ \\
MATERIAL_DEF & FUEL U235A & $2.5533 \mathrm{E}-3$ \\
MATERIAL_DEF & FUEL U236A & $9.5684 \mathrm{E}-6$ \\
MATERIAL_DEF & FUEL U238A & $1.5316 \mathrm{E}-4$ \\
MATERIAL_DEF & FUEL B10A & $1.5539 \mathrm{E}-4$ \\
MATERIAL_DEF & FUEL B11A & $6.2547 \mathrm{E}-4$ \\
MATERIAL_DEF & FUEL C12A & $1.9521 \mathrm{E}-4$ \\
MATERIAL_DEF & FUEL ALA & $5.2751 \mathrm{E}-2$ \\
\hline
\end{tabular}

Figure 5. Material Definition in the Assignment File (Atom Fractions).

Figure 6 demonstrates the definition of a material called Water from 2 compositions appearing in the cross section file, "H_ _ 1 " and "O__16". Again, the MATERIAL_DEF keyword is used. The negative fraction indicates that the value is given in terms of weight fraction rather than atom fraction.

\begin{tabular}{|lll|}
\hline MATERIAL_DEF Water $\mathrm{H}$ & 1 & -0.1121 \\
MATERIAL_DEF Water $0 \_16$ & -0.8879 \\
\hline
\end{tabular}

Figure 6. Material Definition in the Assignment File (Weight Fractions).

Figure 7 demonstrates the definition of a material called Water from 2 compositions appearing in the cross section file, "H1" and "O16", as well as the definition of a material called Salt from compositions "NA23" and "Chlor". The fractions are assumed to be atom fractions 
since they are positive. The final line then defines a mixture called Saline which is $0.9 \%$ Salt and 99.1\% Water by atom fraction. This example demonstrates the recursivity and flexibility of the MATERIAL_DEF keyword.

\begin{tabular}{|lll|}
\hline MATERIAL_DEF Water H1 & 2.0 \\
MATERIAL_DEF Water O16 & 1.0 \\
& & \\
MATERIAL_DEF Salt NA23 & 1.0 \\
MATERIAL_DEF Salt Chlor & 1.0 \\
& & \\
MATERIAL_DEF Saline 0.009 Salt 0.991 Water
\end{tabular}

Figure 7. Recursive Material Definition in the Assignment File.

It is not permitted to mix weight fractions and atom fractions in the definition of a material. Also, if a material is defined recursively, all of its precursors must use the same unit for fraction. Taking the example above, you cannot use weight fraction for Water and atom fraction for Salt, whatever your choice of fraction unit for Saline is.

\subsubsection{Assigning Materials to Blocks}

In the previous section, we defined a material called FUEL. We can assign this material (or any other composition appearing in the material assignment file or the cross section file) to a block in the mesh. Say block 111 (block numbering was defined by the user during mesh creation) is supposed to be a fuel pin. We can associate the material FUEL to block 111 using the REGION_ALIAS keyword shown in Figure 8.

REGION_ALIAS REGION_000000111 FUEL

Figure 8. Assigning a Material to a Block in the Assignment File.

Note the numbering convention used in PROTEUS-SN to refer to blocks. Block 111 is addressed as REGION_000000111 in the PROTEUS-SN assignment file. Block numbers in the mesh must therefore be no more than 9 digits in order to avoid possible duplicate character strings (limited to 9 digits).

\subsubsection{Assigning Block Properties}

We have not yet accounted for the actual material density of the block, or any other properties. The previous step simply linked cross section data to a specific region. If macroscopic cross section data was provided, no further action is necessary since the number density is incorporated into the data. More typically, the cross section data file contains microscopic cross section data and the density must be assigned. Figure 9 demonstrates the assignment of block 
111 's total atom density to 5.6129E-02 atoms/barn-cm using the keywords REGION_PROPERTY and ATOM_DENSITY.

\section{REGION_PROPERTY REGION_000000111 ATOM_DENSITY 5.65129E-02}

Figure 9. Assigning Total Atom Density to a Block in the Assignment File.

Alternatively, the total mass density in $\mathrm{g} / \mathrm{cm}^{3}$ can be assigned to a block as shown in Figure 10. When using the Density (g/cc) property, PROTEUS uses the mass specified in the ISOTXS file for each basic component to convert to number density.

\section{REGION_PROPERTY REGION_000000111 Density(g/cc) 8.595}

Figure 10. Assigning Total Mass Density to a Block in the Assignment File.

The keyword REGION_PROPERTY is therefore used to assign various properties to the mesh such as ATOM_DENSITY, Density $(\mathrm{g} / \mathrm{cc})$, TEMPERATURE(K), and MATERIAL_MODEL to each mesh region. This approach is similar to MCNP where the same material can be used for multiple regions but at different concentrations. For the standard PROTEUS-SN user, the only properties of interest are Density $(\mathrm{g} / \mathrm{cc})$ and ATOM_DENSITY.

An example of a complete assignment file for a fictitious problem is shown in Figure 11. This assignment file assigns materials to two blocks (block 1 and block 7).

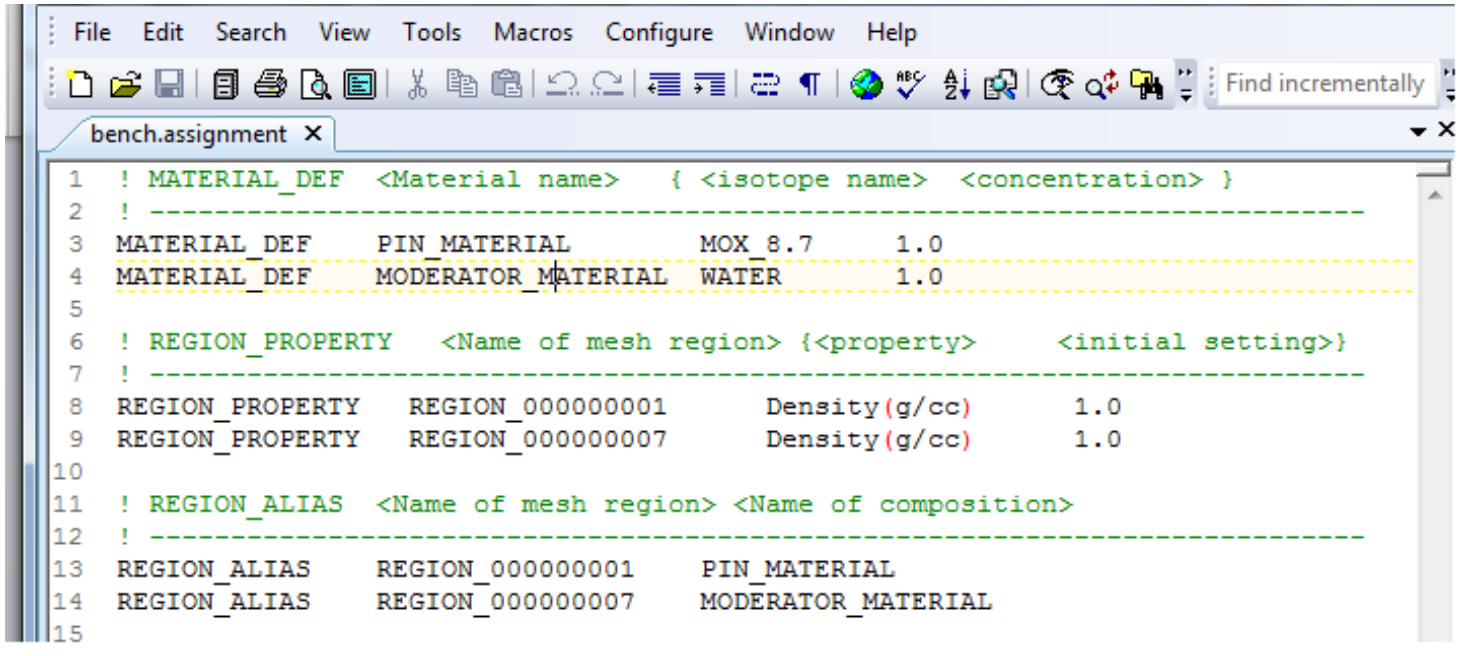

Figure 11. Example PROTEUS-SN Assignment File. 


\subsection{Mesh Partitioning File (*.partitioning)}

As mentioned, PROTEUS performs parallel decomposition in angle and/or space. Spatial domain decomposition for finite element methods consists of breaking the mesh into contiguous smaller pieces of mesh, each piece having approximately the same number of degrees of freedom. Each small piece of the mesh is assigned to a given processor as its work (part of the global vector space). The mesh partitioning procedure is performed in PROTEUS-SN via METIS. Mesh partitioning can be done (1) dynamically at execution (note that the version of METIS we are connected to limits this work to serial) or (2) in advance by providing an additional input partitioning file. Note that PROTEUS-SN always outputs a partitioning file with the extension ".partitioning" in case (1).

For machines with considerable memory resources, users typically find it easier to provide a standard mesh file and just have PROTEUS-SN compute the partitioning at runtime. However, in cases where the user will be re-using the partitioned mesh, or where the entire mesh cannot actually fit into memory (i.e. on low memory machines such as BlueGene/P hardware), the mesh partitioning has to be done in advance. The pre-partitioned mesh file (*.partitioning) is the same as what PROTEUS-SN would partition during normal execution. Note that a valid SOURCEFILE_MESH must be provided that is consistent with the partitioning file. The path to the partitioning file is specified in the driver input file using the keyword SOURCEFILE_MESHPART as seen earlier in Table 4.

If no partitioning file is given or if the partitioning file is not valid (it does not match the spatial decomposition), PROTEUS will create a new partitioning file. To create a partitioning file of your own choosing, you can run a calculation without a partitioning file and then keep the created partitioning file for other calculations with the same mesh and spatial decomposition (only the angular or the group structures vary). It is also possible to obtain a mesh partitioning file without solving the whole problem by using the keyword MESHSIMULATE. If MESHSIMULATE is set to a non-zero positive value, a serial PROTEUS job will load the mesh and generate the partitioning file for a spatial decomposition corresponding to the number given in MESHSIMULATE. The file will be exported with the name "LegacyConversion.pntmesh.partitioning."

The BGPmesh partitioned mesh file format was specifically constructed to minimize the memory overhead to accommodate the BlueGene/P machine. It is not supported in this release, and we suggest contacting nera-software@anl.gov for any questions or concerns with partitioning on low-memory machines.

\subsection{Fixed Source File (*.fixeds)}

To describe a problem with a fixed source contribution, the input option SOURCEFILE_FIXEDSOURCE can be used to specify the path to the fixed source definition file. Three types of fixed sources are supported, all of which are isotropic in angle: vertex-based, mesh region-based (flat in space), and coordinate point based (assuming there is a vertex at the coordinate). In all cases, one should define fixed source information in the fixed source file for 
each and every energy group using Table 5 as a reference. Note that any combination of options in the table can be used simultaneously in the file in an additive sense

Table 5. Fixed Source File Format

\begin{tabular}{|c|c|c|}
\hline Keyword & Input Data & Description \\
\hline PICK_VERTEX & $<$ VertexID $><$ Group1 $><$ Group2 $>$... $<$ GroupG $>$ & $\begin{array}{l}\text { Selects the vertex and the } \\
\text { magnitude of the isotropic } \\
\text { source to apply for each } \\
\text { energy group. }\end{array}$ \\
\hline PICK_REGION & $<$ NAME $><$ Group1 $><$ Group2 $>$... $<$ GroupG $>$ & $\begin{array}{l}\text { Selects a region name, i.e. } \\
\text { "REGION_0000001" } \\
\text { specified in the } \\
\text { assignment file and the } \\
\text { magnitude of the source } \\
\text { to apply for each energy } \\
\text { group. Source is isotropic } \\
\text { in angle and flat in space. }\end{array}$ \\
\hline PICK_COORDINATE & $<\mathrm{X}\rangle\langle\mathrm{Y}\rangle\langle\mathrm{Z}\rangle\langle$ Group1 $><$ Group2 $>$... $<$ GroupG $>$ & $\begin{array}{l}\text { Selects a coordinate in } \\
\text { space (a mesh vertex must } \\
\text { lie at this position) and the } \\
\text { magnitude of the fixed } \\
\text { source to apply for each } \\
\text { energy group. }\end{array}$ \\
\hline
\end{tabular}

\subsection{Kinetics Input File (kinetics.inp)}

The PROTEUS-SN distribution includes a basic time-dependent capability using the adiabatic approximation (simplification to the quasi-static approximation). In addition to the four standard input files, kinetics calculations require an additional kinetics input file placed in the working directory. Additionally, the kinetics capability allows different assignment files to be used at each timestep.

PROTEUS-SN automatically searches for a kinetics input file called "kinetics.inp". To use a different kinetics input file name, PROTEUS-SN must be executed using the command line option "-kinetics $<$ filename $>$ ".

Table 6 lists all of the input options used in the kinetics input file.

Table 6. Kinetics Input Options.

\begin{tabular}{|l|l|l|l|}
\hline Keyword & Input Data & Default value & Description \\
\hline
\end{tabular}




\begin{tabular}{|c|c|c|c|}
\hline Keyword & Input Data & Default value & Description \\
\hline \multicolumn{4}{|c|}{ Kinetics Solver Options } \\
\hline UPDATE_LAMBDABETA & $0,1,2$ & 0 & $\begin{array}{l}0=\text { Update } \Lambda \text { and } \beta \text { at } \\
\text { each time step using } \\
\text { time-dependent adjoint } \\
\text { flux } \\
1=\text { Update } \Lambda \text { and } \beta \text { at } \\
\text { each time step using the } \\
\text { initial condition adjoint } \\
\text { flux } \\
2=\text { Do not update } \Lambda \text { and } \\
\beta \text { at each time step, } \\
\text { instead use initial } \\
\text { condition values } \\
\text { (Note: Older versions of } \\
\text { the code use YES or NO } \\
\text { for this variable to } \\
\text { correspond to option } 0 \\
\text { and } 2 \text {, respectively) }\end{array}$ \\
\hline USE_RADAU & YES or NO & YES & $\begin{array}{l}\text { YES = Use the Radau } \\
\text { solver (default, more } \\
\text { accurate) } \\
\text { NO = Use the traditional } \\
\text { finite difference solver }\end{array}$ \\
\hline $\begin{array}{l}\text { USE_FIXEDSOURCE } \\
\text { _ADJOINT }\end{array}$ & YES or NO & NO & $\begin{array}{l}\text { YES = Use the fixed } \\
\text { source adjoint } \\
\text { NO = Use the } \\
\text { homogeneous adjoint }\end{array}$ \\
\hline \multicolumn{4}{|c|}{ Output File Options } \\
\hline EXPORT_PRECURSORS & $<$ Path to file $>$ & - & $\begin{array}{l}\text { Output file for precursor } \\
\text { and power data at each } \\
\text { coarse time step. }\end{array}$ \\
\hline INITIAL_EXPORT_FILE & $<$ Path to file $>$ & initial.pmo & $\begin{array}{l}\text { Output file for the full flux } \\
\text { solution at the initial } \\
\text { timestep. }\end{array}$ \\
\hline $\begin{array}{l}\text { INITIAL_REGIONEDITS } \\
\text { _FILE }\end{array}$ & $<$ Path to file $>$ & initial.out & $\begin{array}{l}\text { Output file for the block- } \\
\text { wise region edits at the } \\
\text { initial timestep. }\end{array}$ \\
\hline \multicolumn{4}{|c|}{ Input File Options } \\
\hline
\end{tabular}




\begin{tabular}{|c|c|c|c|}
\hline Keyword & Input Data & Default value & Description \\
\hline INITIAL_MATERIAL_FILE & $<$ Path to file $>$ & initial.assignment & $\begin{array}{l}\text { Input assignment file for } \\
\text { the initial timestep. } \\
\text { Future timesteps will use } \\
\text { the assignment files listed } \\
\text { in TIME_STEP input. }\end{array}$ \\
\hline $\begin{array}{l}\text { INITIAL_FIXEDSOURCE } \\
\text { _FILE }\end{array}$ & $\begin{array}{c}<\text { Path to file }> \\
\text { or } \\
\text { NULL }\end{array}$ & initial.fixeds & $\begin{array}{l}\text { Input fixed source file for } \\
\text { the initial time step. Use } \\
\text { NULL if no fixed source } \\
\text { input. } \\
\text { Future timesteps will use } \\
\text { the fixed source files } \\
\text { listed in TIME_STEP input. }\end{array}$ \\
\hline TIME_STEP & $\begin{array}{c}<\text { time }><\text { intervals }> \\
<* \text {.assignment }><* \text {.fixeds }> \\
<* \text {.h5 }><* \text { out }>\end{array}$ & - & $\begin{array}{l}\text { Specifies time step } \\
\text { information and inputs. } \\
\text { Repeat this card input for } \\
\text { each timestep. } \\
\text { Required: } \\
<\text { time }>\text { real time at end } \\
\text { of the timestep } \\
<\text { interval }>\text { number of } \\
\text { intervals to use (report } \\
\text { solution at) for this } \\
\text { timestep } \\
<* \text {.assignment }>= \\
\text { assignment file to use for } \\
\text { this timestep } \\
\text { Optional (can appear in } \\
\text { any order): } \\
<* \text { fixeds }>\text { fixed source } \\
\text { file to use or NULL } \\
<* \text {.h5 or *.pmo }>\text { flux } \\
\text { solution output } \\
<* . \text { out }>=\text { region edits } \\
\text { output }\end{array}$ \\
\hline DELAY_XS_FILE & $<$ Path to file $>$ & default.dlayxs & $\begin{array}{l}\text { Input delay cross section } \\
\text { data file (in DLAYXS } \\
\text { format). }\end{array}$ \\
\hline
\end{tabular}




\begin{tabular}{|c|c|c|l|}
\hline Keyword & \multicolumn{1}{|c|}{ Input Data } & Default value & \multicolumn{1}{c|}{ Description } \\
\hline & & & $\begin{array}{l}\text { Maps the isotope name in } \\
\text { ISOTXS to the delay xs } \\
\text { isotope name in DLAYXS, } \\
\text { in case they differ. }\end{array}$ \\
\hline DELAY_ALIAS & $\begin{array}{l}\text { <ISOTXS_XS_NAME> } \\
\text { <DELAY_XS_NAME> }\end{array}$ & - & $\begin{array}{l}\text { Repeat this card input for } \\
\text { each delay alias. }\end{array}$ \\
\hline
\end{tabular}

The adiabatic approximation to the quasi-static kinetics method essentially computes solutions to the point-kinetics equations at every time step. However, the kinetics parameters ( $\Lambda$ and $\beta$ ) are a function of the time-dependent forward and adjoint flux. In this approximation, the basic "steady state" forward and adjoint flux are calculated at every time step for updated material properties and used to update $\Lambda$ and $\beta$ if UPDATE_LAMBDABETA 0 is requested. If UPDATE_LAMBDABETA 1 is requested, the parameters are updated using the updated forward flux but only the initial condition adjoint flux. If UPDATE_LAMBDABETA 2 is requested, the parameters are calculated once at the initial condition and never updated.

The keyword USE_RADAU indicates whether the Radau solver should be used to solve the point kinetics equations. The default value is yes. The Radau solver is based on the Runge-Kutta method and is more accurate than the implicit differencing scheme that would otherwise be used. To disable the Radau solver and instead use the finite differencing scheme, USE_RADAU NO should be specified.

For fixed source problems, the keyword USE_FIXEDSOURCE_ADJOINT specifies whether the fixed source adjoint flux should be used in updating $\Lambda$ and $\beta$ (YES) or whether the homogeneous adjoint flux solution should be used (NO).

The delay cross section data (decay constants, emission spectra and delayed precursor yields) must be provided in the DELAY_XS_FILE input in DLAYXS file format (available from MC ${ }^{2}$ 3). Material assignment files for each timestep must be provided in the INITIAL_MATERIAL_FILE and TIMESTEP inputs. The DELAY_ALIAS input is used to map the isotope name in the cross section file to delay cross section data in the DLAYXS file, in case the files contain different user-specified names for the same isotopes.

The TIMESTEP card specifies information about each timestep. This card is used to specify the real time at the end of the timestep, how many intervals to perform, and the material assignment file. Optionally, a fixed source input file (*.fixeds) and flux (*.h5 or *.pmo) and region edit (*.out) output files for the timestep may also be specified (in any order) using filenames with the appropriate suffixes. An interval is like a sub-timestep within a timestep. At the end of an interval, only the point kinetics equations are solved for power, etc using the 
previous interval values for $\Lambda, \beta$ and the eigenvalue. At the end of a timestep, the transport equation (forward and adjoint) are solved to obtain a new $\Lambda, \beta$ and eigenvalue. These are then used as updated parameters in the point kinetics equations. Flux files are provided only at the end of a timestep.

The precursor densities and power data at each coarse time step can be output to a file specified by the EXPORT_PRECURSORS keyword. The block-wise region edits (group scalar flux, power, absorption, volume) can be exported to the file specified by INITIAL_REGIONEDITS_FILE (for the initial condition) and to separate files for each coarse timestep specified in the TIMESTEP input. Similarly, the full flux solution can be exported to INITIAL_EXPORT_FILE (for the initial condition) and to separate files for each coarse timestep specified in the TIMESTEP input.

Figure 12 shows an example kinetics.inp file with 3 timesteps beyond the initial configuration. The kinetics parameters will be updated at every time step. The alias for isotope P239H (ISOTXS) is PU239 (DLAYXS), meaning that the PU239 delay cross section data will be mapped to isotope $\mathrm{P} 239 \mathrm{H}$.

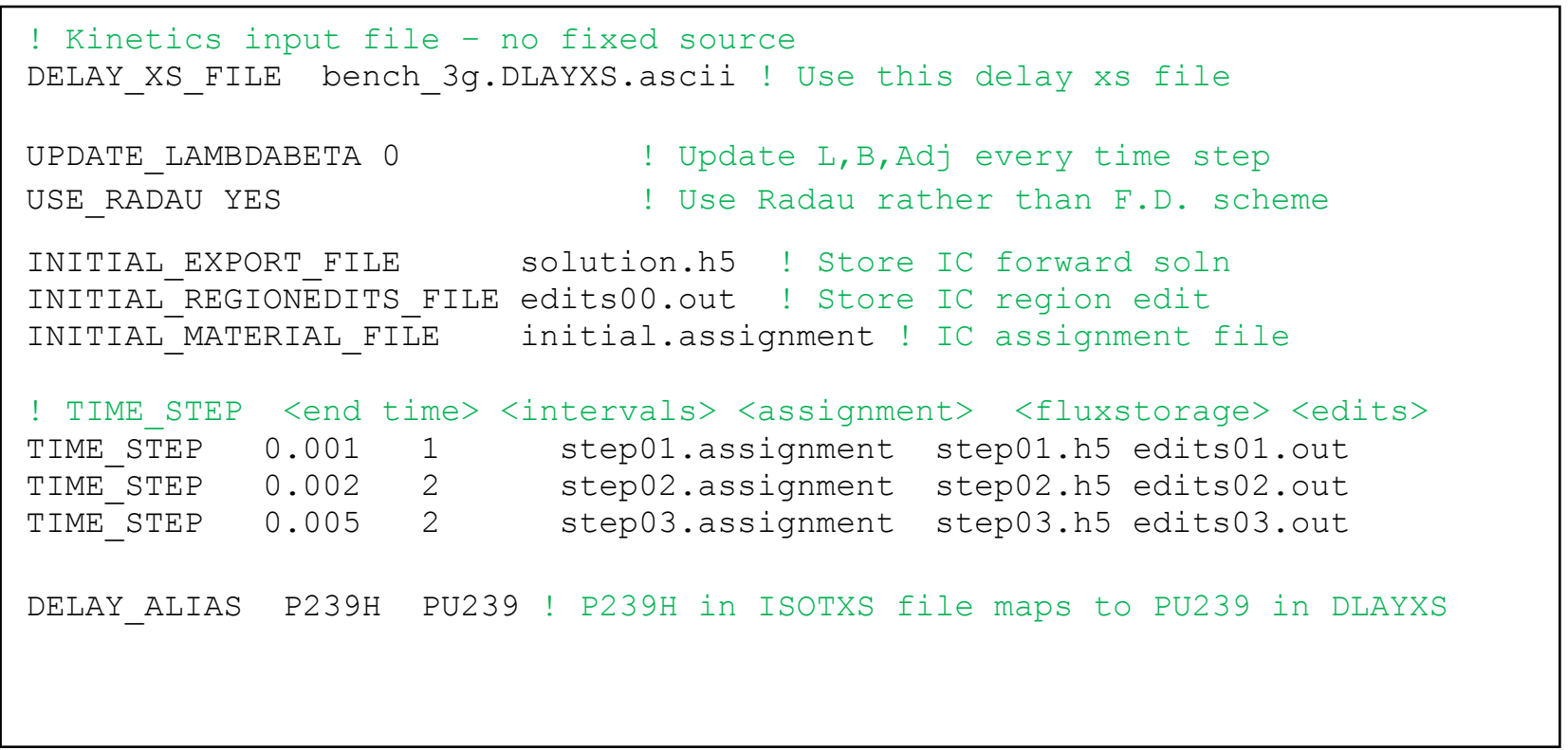

Figure 12. Example Kinetics Input File with No Fixed Source.

Figure 13 is an example of a kinetics input file with fixed source. Note that for fixed source problems, a fixed source file should be specified for all timesteps including initial condition. The value NULL can be supplied in place for timesteps without a fixed source. 


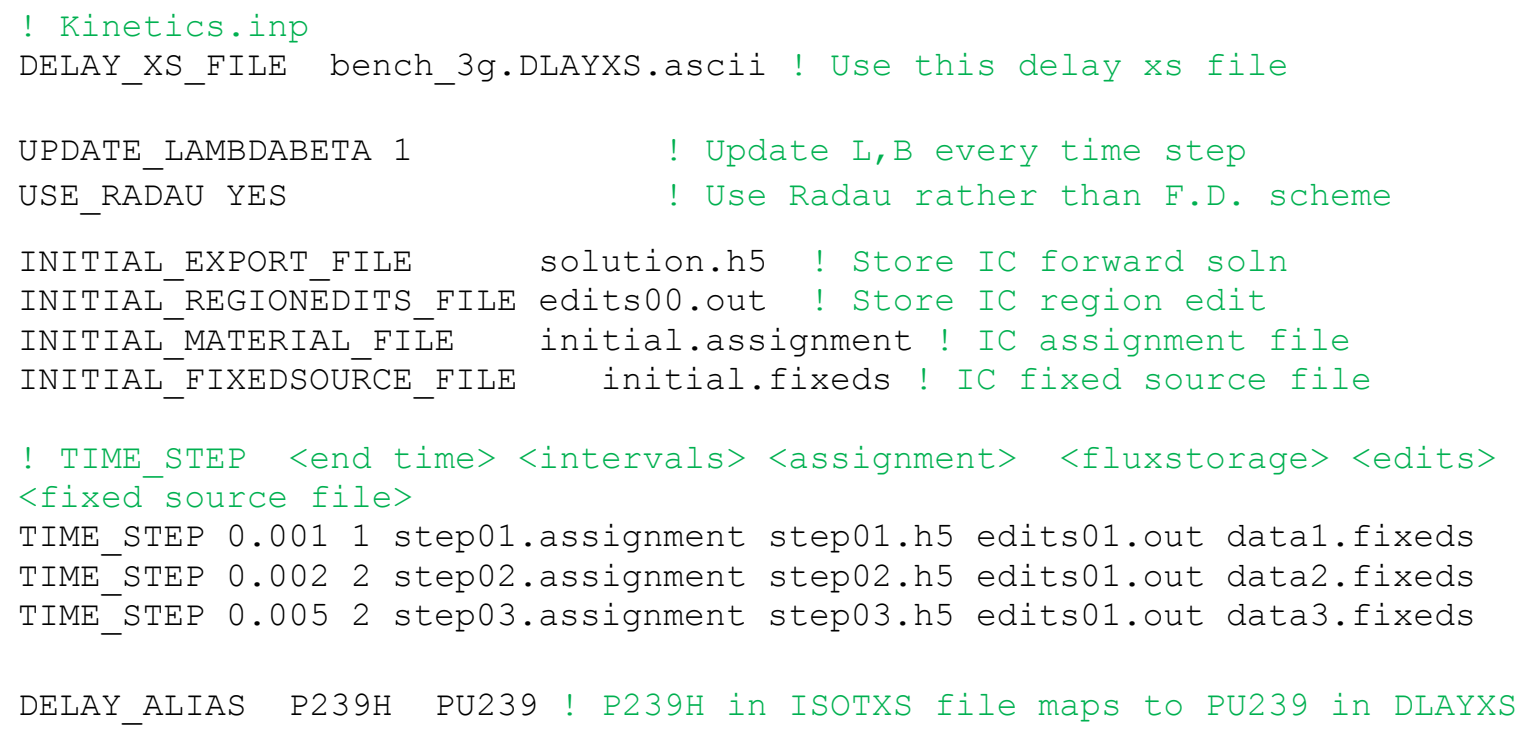

Figure 13. Example Kinetics Input File with Fixed Source. 


\section{Output}

PROTEUS-SN has four basic types of output: (1) main output text file, (2) full solution, (3) VTK files for mesh and cubature, and (4) volume-integrated region edits. The main output text file is always produced upon execution. The other three types of output are optionally triggered by keywords EXPORT_FLUX, EXPORT_MESH, and EXPORT_CUBATURE, and EXPORT_EDITS in the driver input file.

\subsection{Main Output File}

The main text-based output is printed to standard output or to a file if the -output option is used. The output contains confirmation that the input was imported successfully, parallel timing summaries, and eigenvalue iteration history results.

Appendix F provides an example output file. At the top of the file, the various input options are echoed and errors are produced if the input files were not successfully imported. This section is followed by the outer iteration history which reports the eigenvalue (and other quantities) as the calculation proceeds. At the end of the outer iteration history, the final eigenvalue is reported along with an error estimate. At the end of the output file, an MPI timing history provides an overview of where time was spent in the calculation.

\subsection{Full Solution}

The full solution in the entire domain can optionally be exported to an ASCII file (.pmo) or to HDF5 file format (.h5). We recommend using the .h5 format which requires that PROTEUS-SN be compiled with HDF5. The creation of the HDF5 or PMO files is triggered by the EXPORT_FLUX and EXPORT_FILE options in the input driver file, for example:

$\begin{array}{ll}\text { EXPORT_FLUX } & \text { YES } \\ \text { EXPORT_FILE } & \text { abtr_L5T7.h5 }\end{array}$

These two lines will ensure that a file named "abtr_L5T7.h5" is created, in HDF5 file format, containing the full flux solution. The .h5 output file includes the full 3D mesh, composition, scalar flux by group, nu*fission rate, absorption rate, and power. Temperature and density are not recorded. Also, the eigenvalue is not recorded in this file.

The .h5 file can be opened and viewed in VisIt as a "UNIC" type file in the drop-down list. Unfortunately, PROTEUS does not output integrated quantities such as pin power or assemblytotal power at this time. Various techniques are available in VisIt to post-process the output, such as summing the solution in a certain region, or exporting values along a line using the "Line-out" analysis option. To use the Line-out analysis tool in VisIt, the user must click on visit's "Analysis" menu, choose Line-out, specify two points on the line, and export the database as a Curve file.

Figure 14 through Figure 16 show PROTEUS-SN results visualized with VISIT using the PROTEUS plugin. Quantities such as the mesh, power distribution, and flux distribution for each energy group can be visualized. 

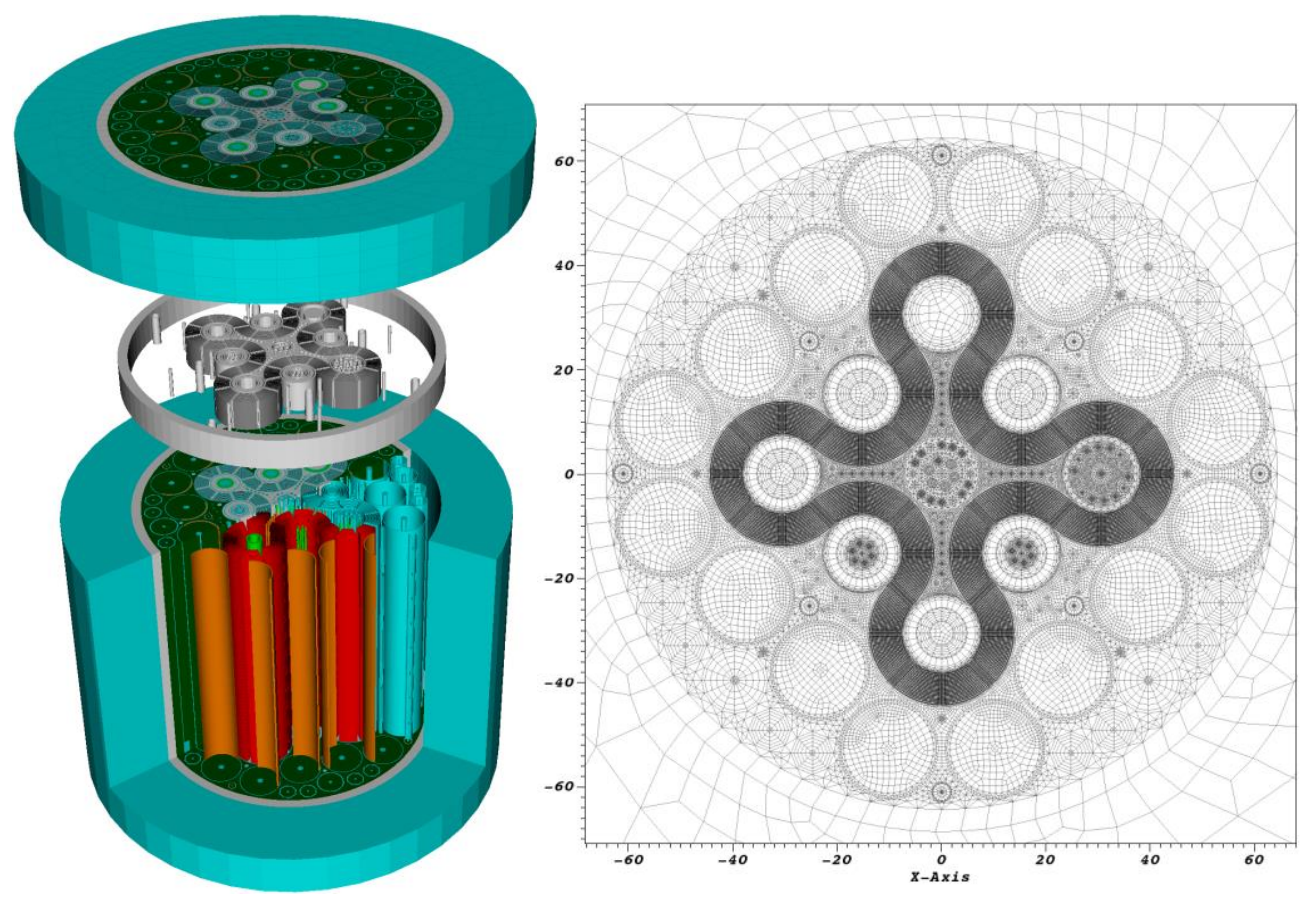

Figure 14. Visualization of Geometry and Mesh Used to Analyze the Advanced Test Reactor.

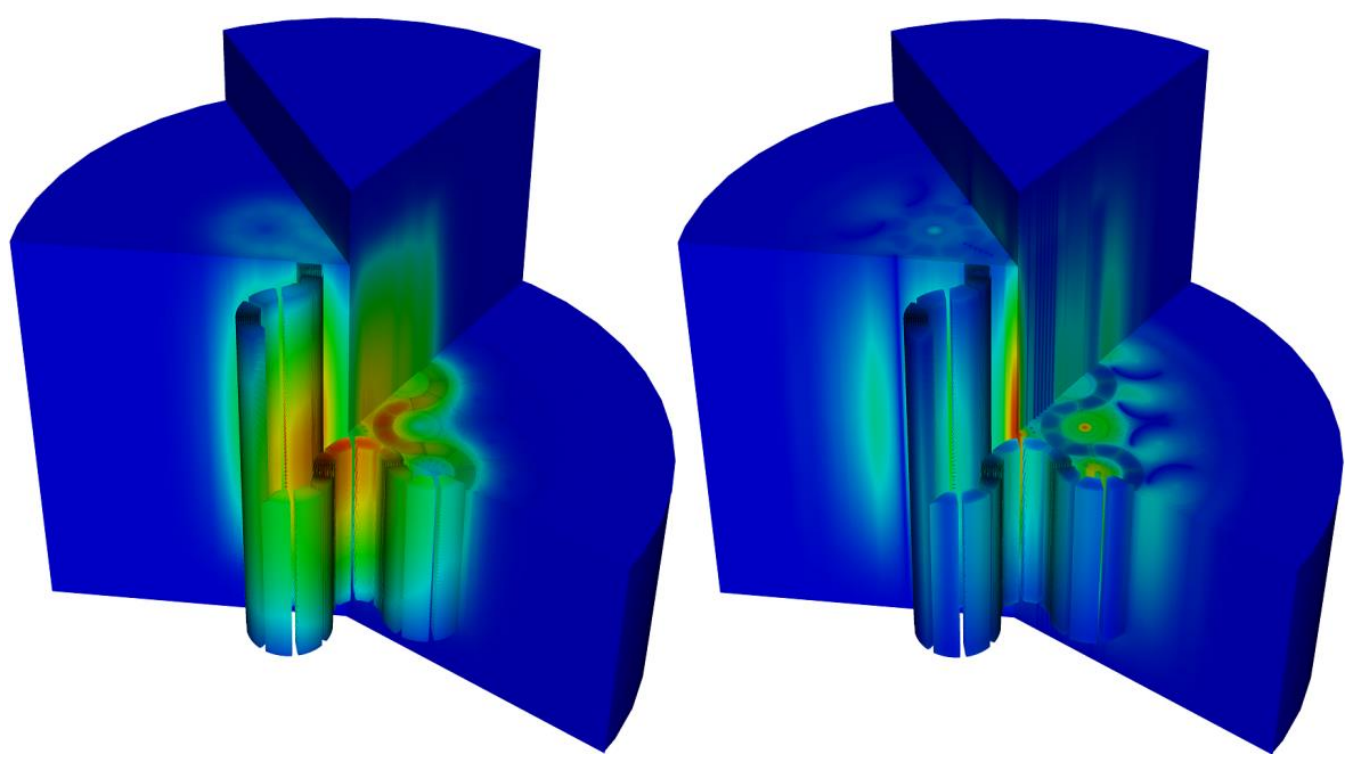

Figure 15. ATR fast (left) and Thermal (right) Flux Calculated by PROTEUS-SN. 

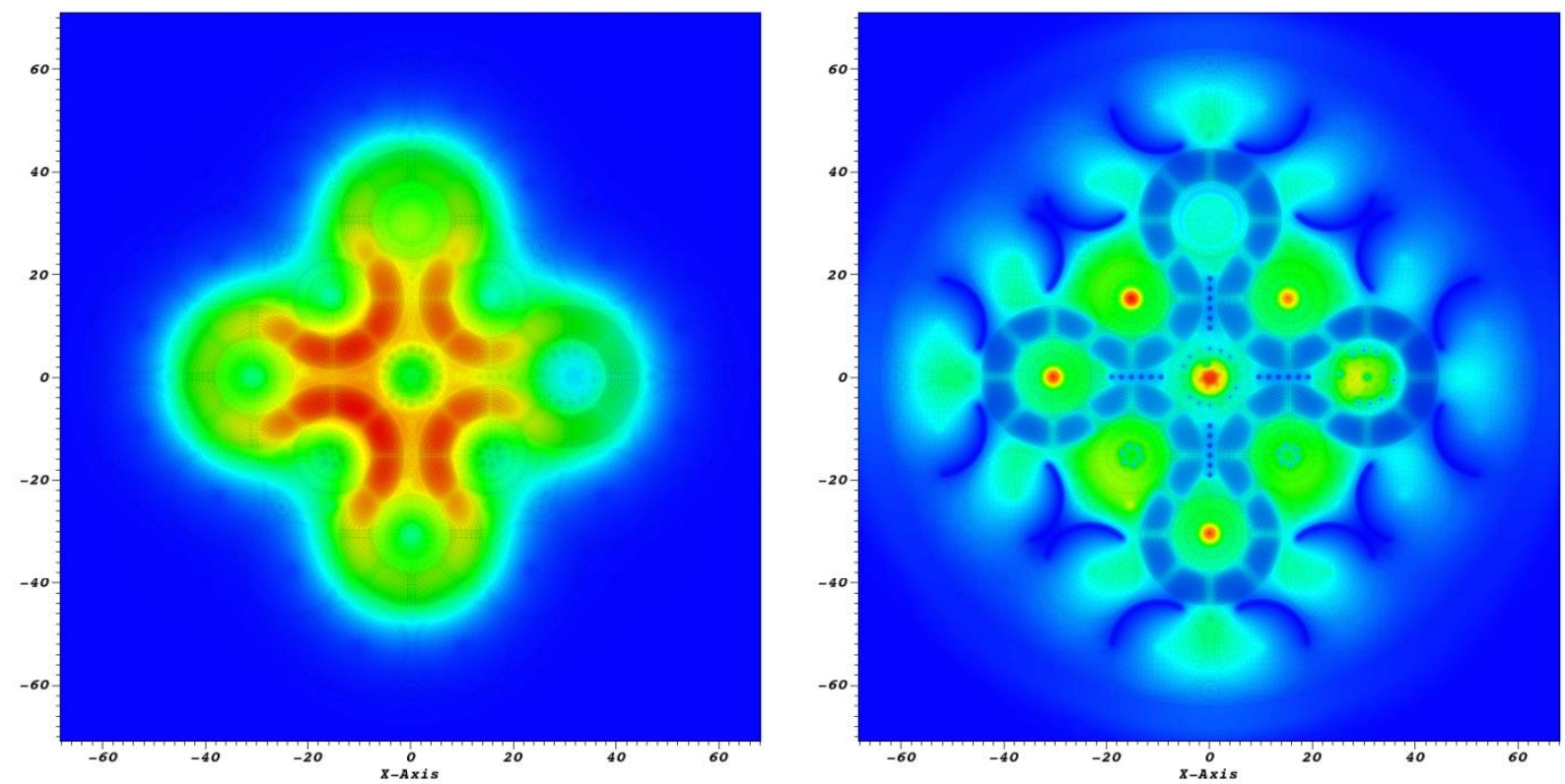

Figure 16. Visualization of 2D Fast (Left) and Thermal (Right) Fluxes for ATR Calculation.

If the output was requested in .pmo format, (.pmo recommended for supercomputers/ extremely large problems), several .pmo files will be produced in the output directory. These must first be converted to .h5 format using the PMO_AssemblyHDF5.x converter provided with PROTEUS-SN (see Section 3.1.4 Converting PMO to HDF5 Output).

\subsection{VTK Files for Mesh and Cubature}

The mesh can also be visualized using the EXPORT_MESH option to produce a VTK file viewable in VisIt or another VTK-friendly visualization program. This option can be invoked to visually inspect the mesh, for example. Note that the mesh is also included in the full solution output.

The angular cubature can be visualized using the EXPORT_CUBATURE option to produce a VTK file. It can be helpful to visualize the placement of angles, and associated weights for each ordinate, on the unit sphere.

\subsection{Region Edit Integrals}

The region edit integrals can also be exported to a simple text file for quick analysis using the EXPORT_EDITS option and specifying the name of the desired output file. The resulting file will contain a list of quantities by block number (region number) specified in the mesh: volume, power, fission, absorption, and flux by group. Keep in mind that the total power will be normalized to the user-specified value of THERMAL_POWER (excludes fixed source case). 


\section{Example Problem}

Here we discuss a simple example taken from the PROTEUS-SN benchmark 5 test case. This test case is a steady-state forward and adjoint calculation for a $2 \mathrm{D} 1 / 8^{\text {th }}$ pin cell with reflected boundaries. The materials in the problem are MOX fuel and water. As with any deterministic calculation, a space-angle-convergence study should be performed to assess convergence (not shown here). The following input files are given for this problem:

- bench05.inp (driver input)

- bench.nemesh (mesh)

- bench.anlxs (cross sections)

- bench.assignment (material assignment file)

Examining the driver input file in Figure 17, we see that the paths to the mesh, cross section data, and material assignment file are clearly defined by the SOURCEFILE_MESH, SOURCEFILE_XS, and SOURCEFILE_MATERIAL keywords. The Solve_Type keyword indicates that both the forward and adjoint flux are to be solved (this is the default value of Solve_Type, so this value is not required). A Legendre-Tchebychev cubature of order L3T9 will be used, for a total of $1 / 2(\mathrm{~L}+1)(\mathrm{T}+1)$ or 20 angles in 2D PROTEUS-SN.

The code will attempt to perform maximum parallelization in angle (SEGMENT ANGLE assigned to 0 ). If we only perform the problem in serial, no parallelization will occur. The power will renormalized to 1 Watt (default). The SSOR preconditioner (default) will be used and Tchebychev acceleration of the fission source will be applied (default). The relative error tolerances have been somewhat tweaked. Scattering will be isotropic. The mesh and full solution will be exported to VTK and HDF5 files, respectively. 




Figure 17. Driver Input File for Example Case.

We continue on to the cross section file, specified as bench.anlxs in the driver input. Reviewing the .anlxs file format in Appendix $\mathrm{C}$, we determine that the first line contains the number of compositions, number of energy groups, and another unimportant integer. This file contains 8 compositions with a 7 energy group structure which is listed on the following lines. The composition names are read as UO2_FUEL, MOX_4.3, MOX_7.0, and others (entire file not shown for brevity). Therefore, we should expect to see these composition names in the material assignment file. The multigroup cross section data is listed for each isotope as shown in Figure 18. 


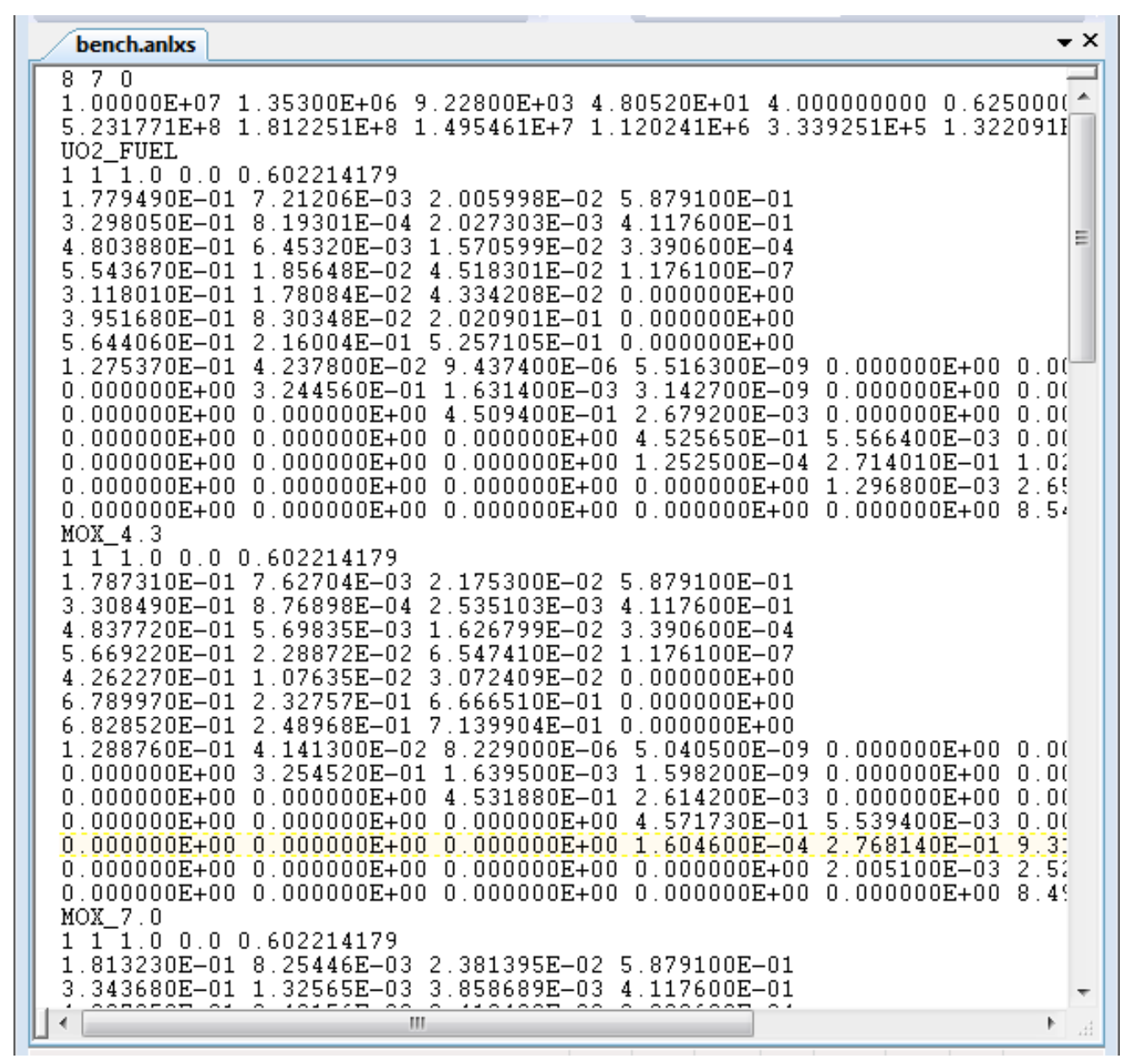

Figure 18. Cross Section File for Example Case.

We also take a quick look at the mesh, provided in bench.nemesh (.nemesh file format described in Appendix D). Card Type 2 lists the number of elements (16), number of vertices (45), and number of boundary surfaces (12).

The first line in card type 3 can be interpreted as "Element 1 has 6 nodes and belongs to block 1". Subsequent lines describe the other elements. Element connectivity follows, as well as a listing of vertex positions and boundary surfaces (not shown for brevity). This is in fact a quadratic-order triangular mesh. 


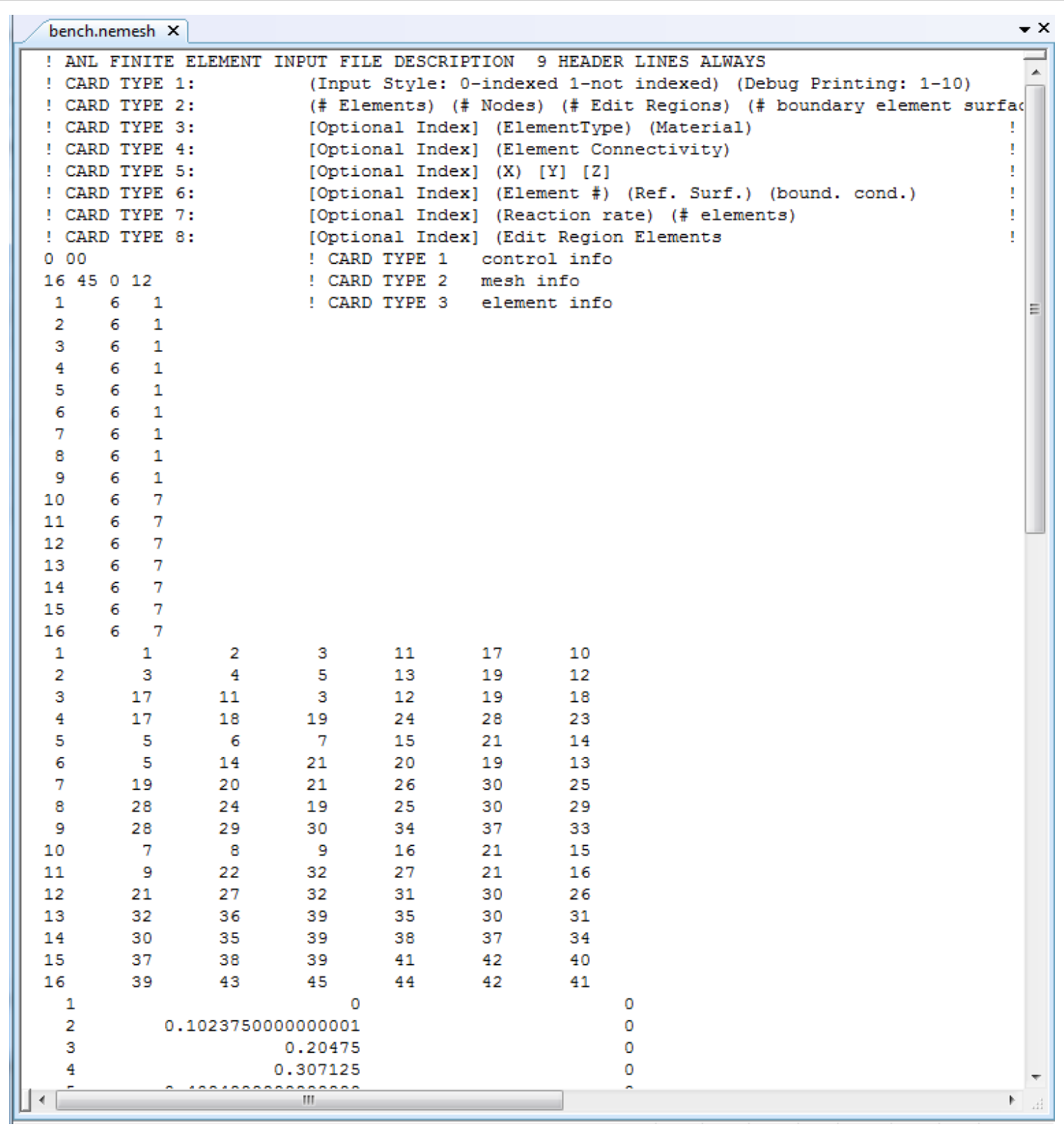

Figure 19. Mesh File for Example Case.

Figure 20 shows the VTK plot of the mesh along with compositions. (The mesh was exported to a VTK file by PROTEUS-SN using EXPORT_MESH.) The block coloring indicates the elements belonging to block 1 (blue) and block 7 (red). 

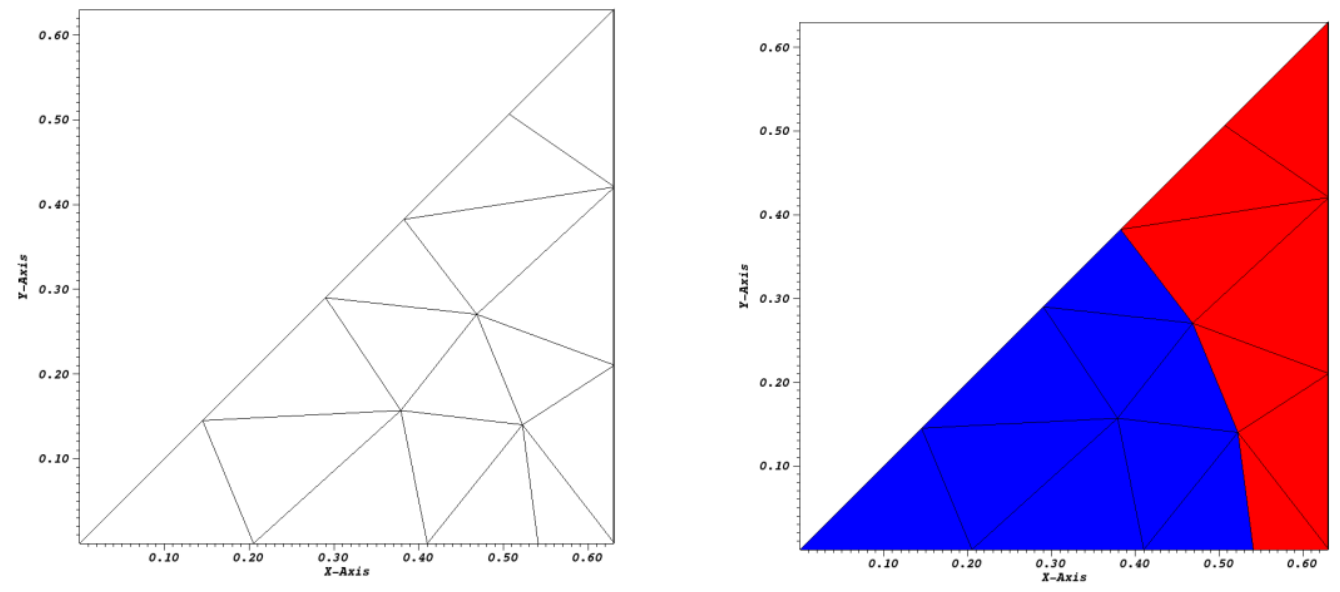

Figure 20. VTK Mesh (left) and VTK Mesh with Block Coloring (right) for Example Case.

A snapshot of the assignment file is shown in Figure 21. The first non-commented line in the file defines a material called PIN_MATERIAL made of 100\% MOX_8.7. The second line similarly defines a material called MODERATOR_MATERIAL made of 100\% WATER. Note that these are actually just aliases to MOX_8.7 and WATER, respectively.

Skipping to the last section, PIN_MATERIAL is assigned to block 1 (blue), and MODERATOR_MATERIAL is assigned to block 7 (red). In the middle section, both blocks are assigned a mass density of $1.0 \mathrm{~g} / \mathrm{cc}$. This mass density will be converted to atom density in the code as shown in Figure 21.

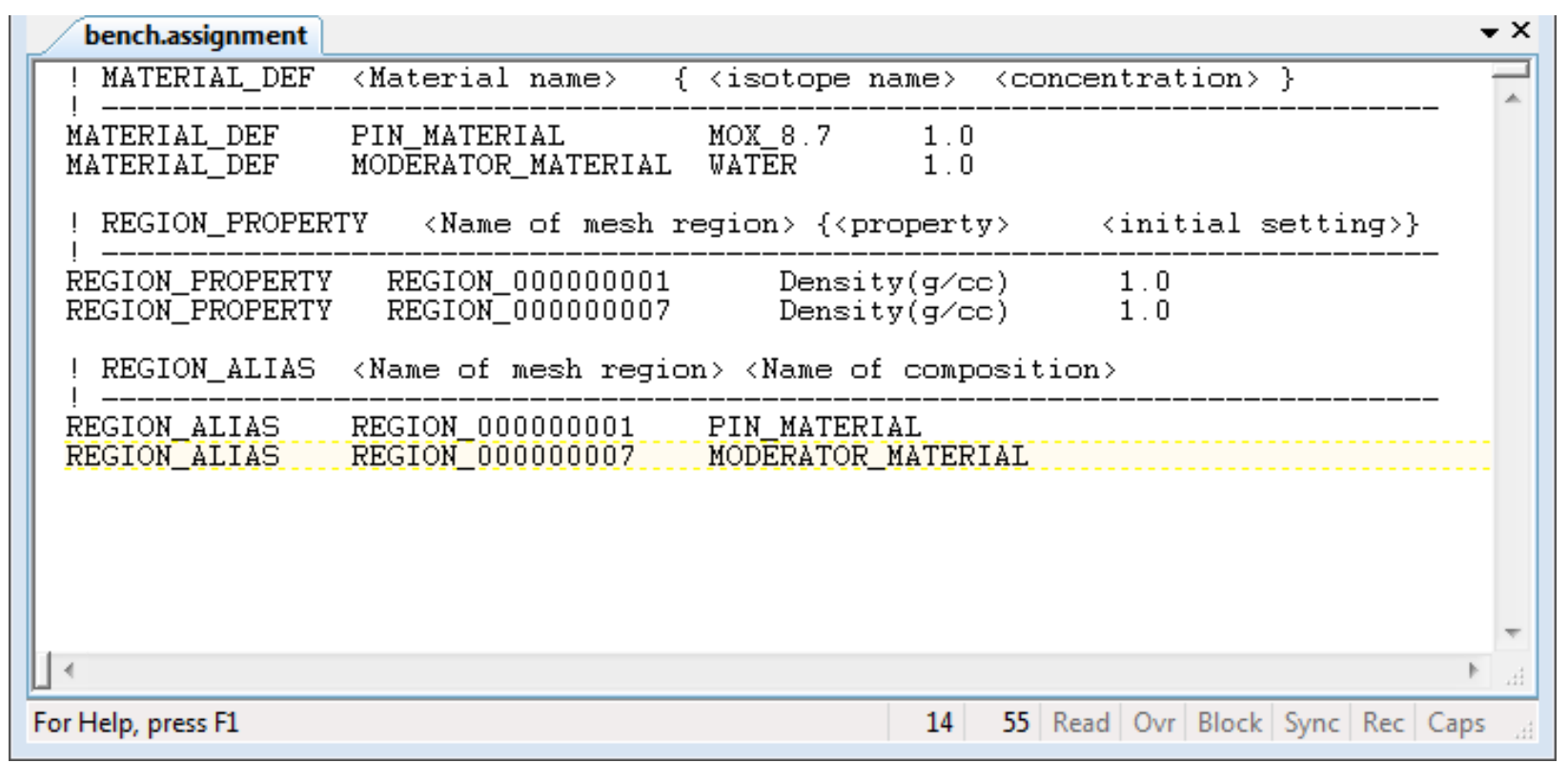

Figure 21. Material Assignment File for Example Case. 
The following command executes the problem in parallel with 4 processors:

\section{mpiexec-n 4 sn2nd.x -input bench05.inp > bench05.out}

We have assumed that the executable and input files all reside in the working directory. After execution, the following files are produced:

- bench05.out

- bench05.h5

- Converted_ANLXSto.ISOTXS

- LegacyConversion.pntmesh

- LegacyConversion.pntmesh.partitioning

The first file, bench05.out, is the main text-based output file containing the eigenvalue. This file is included in Appendix F for reference. The second file, bench05.h5, is the HDF5-formatted output file containing the full solution. The remaining files are the ISOTXS and pntmesh conversions of the .anlxs and .nemesh file, respectively. PROTEUS-SN always converts into these base formats. These files can be saved and re-used in the future instead of using the .anlxs and .nemesh files.

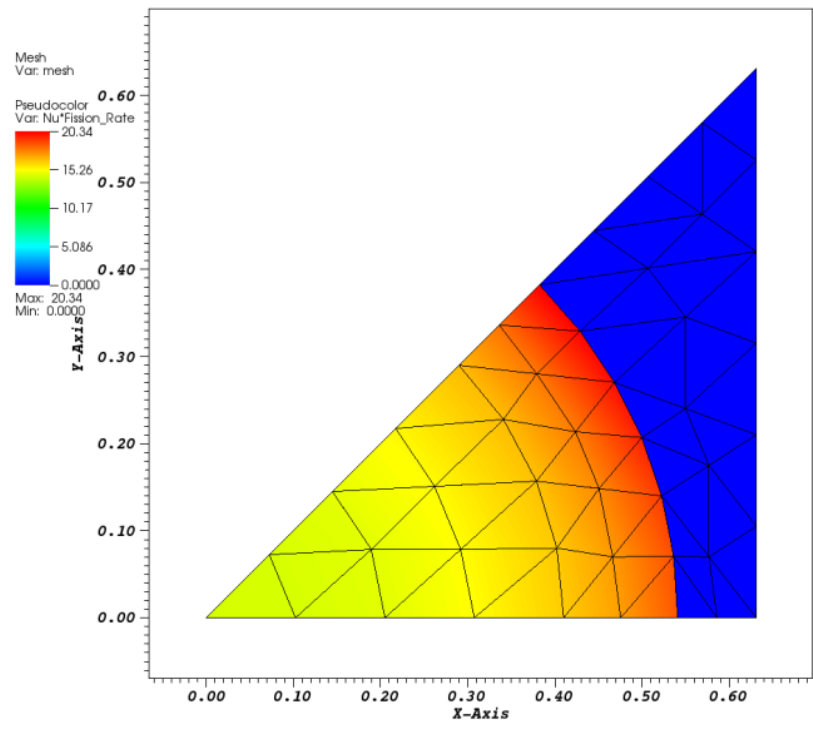

Figure 22. Nu*fission Rate in Test Case Plotted in VisIt.

Importing the .h5 output into visit and plotting the nu*fission rate and the mesh produces a figure like that in Figure 22. Note that VisIt tessellates the quadratic triangular mesh into linear triangles. This solution is also interpolated linearly on the finer mesh rather than quadratically on the original mesh. This limitation is something to consider when using VisIt to plot and obtain higher order solutions. 


\section{Summary}

PROTEUS-SN is a highly scalable, discrete ordinates-based unstructured grid code which solves the even-parity transport equation for nuclear reactor applications. The code is targeted for reactor analysis which requires heterogeneous or unstructured grid geometry representation. The code has been demonstrated to scale to over 200,000 processors and solve problems with nearly 1 trillion space-angle-energy degrees of freedom.

This user manual documents the basics of installing and using PROTEUS-SN. It describes the external library dependencies (MPICH, PETSc, METIS, and HDF5), compilation process, and usage commands. The required input files were also described in detail including examples and file formats. To summarize, PROTEUS-SN requires a finite element mesh, a multigroup cross section data file or a cross section library file, a material assignment file, and a driver input file. For kinetics calculations, an additional kinetics input is required.

Specific tools were recommended for cross section generation, mesh generation, and visualization. For questions or more information on PROTEUS-SN, please contact nerasoftware@anl.gov. 


\section{References}

1. C.H. Lee and W.S. Yang, "MC'-3: Multigroup Cross Section Generation Code for Fast Reactor Analysis," ANL/NE-11-41 (Rev. 1), January, 2012.

2. K.L. Derstine, et al, "DIF3D: A Code to Solve One-, Two-, and Three-Dimensional FiniteDifference Diffusion Theory Problems," ANL-82-64, Argonne National Laboratory, 1984.

3. VisIt User's Manual, Version 1.5, UCRL-SM-220449, October 2005.

4. VTK Web page, www.vtk.org.

5. MPICH Web page, http://www.mpich.org.

6. George Karypis and Vipin Kumar, "A Fast and Highly Quality Multilevel Scheme for Partitioning Irregular Graphs", SIAM Journal on Scientific Computing, Vol. 20, No. 1, pp. 359-392, 1999.

7. METIS Web page, http://glaros.dtc.umn.edu/gkhome/metis/metis/overview

8. S. Balay, J. Brown, K. Buschelman, W. D. Gropp, D. Kaushik, M. G. Knepley, L. Curfman McInnes, B. F. Smith, H. Zhang, PETSc Web page, http://www.mcs.anl.gov/petsc (2013)

9. The HDF Group. Hierarchical Data Format, version 5, 1997-2014. http://www.hdfgroup.org/HDF5/.

10. T.J. Tautges, et al, "MOAB: A Mesh Oriented Database", Report SAND2004-1592, Sandia National Laboratories, April 2004.

11. G. Marleau, R. Roy, and A. Hébert, "DRAGON: A Collision Probability Transport Code for Cell and Supercell Calculations," Report IGE-157, Institut de génie nucléaire, École Polytechnique de Montréal, Montréal, Québec, 1994.

12. H. Childs, et al, "A Contract-Based System for Large Data Visualization", Proceedings of IEEE Visualization 2005, Minneapolis, MN, pp 190-198, 2005.

13. M. A. Smith and E. R. Shemon, "User Manual for the PROTEUS Mesh Tools", ANL-NE15/17 (Rev. 1), Argonne National Laboratory, June, 2015.

14. CUBIT Web page, www.cubit.sandia.gov.

15. NetCDF Web page, http://www.unidata.ucar.edu/software/netcdf/.

16. R. Jain, T.J. Tautges, "NEAMS MeshKit: Nuclear Reactor Mesh Generation Solutions", Proceedings of International Congress on the Advances in Nuclear Power Plants (ICAPP), American Nuclear Society, Charlotte, NC, April 6-9, 2014.

17. T.J. Tautges, R. Meyers, K. Merkley, C. Stimpson, C. Ernst, MOAB: A Mesh-Oriented Database, Sandia National Laboratories report SAND2004-1592, April 2004. 


\section{APPENDIX A. ANGULAR CUBATURES IN PROTEUS-SN}

The following tables describe the resolutions of each angular cubature included with PROTEUS-SN, such as 'CARLSON_EM' which is the conventional level symmetric cubature used in most discrete ordinates codes. In each table, the number of points that appears in $2 \pi$ and $4 \pi$ is given noting that PROTEUS-SN will report having the $2 \pi$ number for $3 \mathrm{D}$ domains (related to symmetry in the even-parity formulation). The "Library Order" specifies what the theoretical spherical harmonics integration capability of each resolution, while the "Order when Error $<10 \mathrm{E}$ 6" column indicates the actual spherical harmonics integration capability. For example, one should not use 'CARLSON_LM' for a problem with $\mathrm{P}_{5}$ anisotropic scattering, since the actual order is stated to be only P3.

The LEG_TCHEBY cubatures are product cubatures and thus require inputs for both PHI_RESOLUTION and THETA_RESOLUTION in the code. PHI_RESOLUTION describes the azimuthal resolution, i.e. resolution in the $\mathrm{x}-\mathrm{y}$ plane. THETA_RESOLUTION describes the polar resolution of the cubature. The resolutions in the table are stated for Resolution=PHI_RESOLUTION=THETA_RESOLUTION, i.e. L1T1, L3T3, etc.

\begin{tabular}{|c|c|c|c|c|c|c|c|c|}
\hline & \multicolumn{4}{|c|}{ CARLSON_EM } & \multicolumn{4}{c|}{ CARLSON_LM } \\
\cline { 2 - 9 } Resolution & $\begin{array}{c}\text { Points } \\
\text { on } 2 \pi\end{array}$ & $\begin{array}{c}\text { Points } \\
\text { on 4 } \pi\end{array}$ & $\begin{array}{c}\text { Library } \\
\text { Order }\end{array}$ & $\begin{array}{c}\text { Order when } \\
\text { Error }<10 \mathrm{E}-6\end{array}$ & $\begin{array}{c}\text { Points } \\
\text { on } 2 \pi\end{array}$ & $\begin{array}{c}\text { Points } \\
\text { on } 4 \pi\end{array}$ & $\begin{array}{c}\text { Library } \\
\text { Order }\end{array}$ & $\begin{array}{c}\text { Order when } \\
\text { Error }<10 \mathrm{E}-6\end{array}$ \\
\hline 1 & 4 & 8 & 2 & 3 & 4 & 8 & 2 & 3 \\
\hline 2 & 12 & 24 & 4 & 5 & 12 & 24 & 4 & 3 \\
\hline 3 & 24 & 48 & 6 & 7 & 24 & 48 & 6 & 3 \\
\hline 4 & 40 & 80 & 8 & 9 & 40 & 80 & 8 & 3 \\
\hline 5 & 60 & 120 & 10 & 11 & 84 & 168 & 10 & 3 \\
\hline 6 & 84 & 168 & 12 & 11 & 84 & 168 & 12 & 3 \\
\hline 7 & 112 & 224 & 14 & 11 & 144 & 288 & 14 & 3 \\
\hline 8 & 144 & 288 & 16 & 11 & 144 & 288 & 16 & 3 \\
\hline
\end{tabular}

\begin{tabular}{|c|c|c|c|c|c|c|c|c|}
\hline & \multicolumn{4}{|c|}{ CARLSON_EQUALW } & \multicolumn{4}{c|}{ PYRAMID_CARLSON } \\
\cline { 2 - 9 } Resolution & $\begin{array}{c}\text { Points } \\
\text { on } 2 \pi\end{array}$ & $\begin{array}{c}\text { Points } \\
\text { on } 4 \pi\end{array}$ & $\begin{array}{c}\text { Library } \\
\text { Order }\end{array}$ & $\begin{array}{c}\text { Order when } \\
\text { Error }<10 \mathrm{E}-6\end{array}$ & $\begin{array}{c}\text { Points } \\
\text { on } 2 \pi\end{array}$ & $\begin{array}{c}\text { Points } \\
\text { on } 4 \pi\end{array}$ & $\begin{array}{c}\text { Library } \\
\text { Order }\end{array}$ & $\begin{array}{c}\text { Order when } \\
\text { Error }<10 \mathrm{E}-6\end{array}$ \\
\hline 1 & 4 & 8 & 2 & 3 & 4 & 8 & 1 & 3 \\
\hline 2 & 12 & 24 & 4 & 5 & 12 & 24 & 3 & 3 \\
\hline 3 & 24 & 48 & 6 & 5 & 24 & 48 & 5 & 5 \\
\hline 4 & 40 & 80 & 8 & 9 & 40 & 80 & 7 & 7 \\
\hline 5 & 60 & 120 & 10 & 11 & 60 & 120 & 9 & 9 \\
\hline 6 & 84 & 168 & 12 & 13 & 84 & 168 & 11 & 11 \\
\hline 7 & 112 & 224 & 14 & 11 & 112 & 224 & 13 & 13 \\
\hline 8 & 144 & 288 & 16 & 17 & 144 & 288 & 15 & 15 \\
\hline 9 & & & & & 180 & 360 & 17 & 17 \\
\hline 10 & & & & & 220 & 440 & 19 & 19 \\
\hline
\end{tabular}




\begin{tabular}{|c|c|c|c|c|c|c|c|c|}
\hline & \multicolumn{4}{|c|}{ LEG-TCHEBY } & \multicolumn{4}{c|}{ X_DIR_LEG-TCHEBY } \\
\cline { 2 - 9 } Resolution & $\begin{array}{c}\text { Points } \\
\text { on } 2 \pi\end{array}$ & $\begin{array}{c}\text { Points } \\
\text { on 4 } \pi\end{array}$ & $\begin{array}{c}\text { Library } \\
\text { Order }\end{array}$ & $\begin{array}{c}\text { Order when } \\
\text { Error }<10 \text {-6 }\end{array}$ & $\begin{array}{c}\text { Points } \\
\text { on } 2 \pi\end{array}$ & $\begin{array}{c}\text { Points } \\
\text { on } 4 \pi\end{array}$ & $\begin{array}{c}\text { Library } \\
\text { Order }\end{array}$ & $\begin{array}{c}\text { Order when } \\
\text { Error < 10E-6 }\end{array}$ \\
\hline 1 & 4 & 8 & 2 & 3 & 4 & 8 & 2 & 3 \\
\hline 2 & 9 & 18 & 4 & 5 & 9 & 18 & 4 & 5 \\
\hline 3 & 16 & 32 & 6 & 7 & 16 & 32 & 6 & 7 \\
\hline 4 & 25 & 50 & 8 & 9 & 25 & 50 & 8 & 9 \\
\hline 5 & 36 & 72 & 10 & 11 & 36 & 72 & 10 & 11 \\
\hline 6 & 49 & 98 & 12 & 13 & 49 & 98 & 12 & 13 \\
\hline 7 & 64 & 128 & 14 & 15 & 64 & 128 & 14 & 15 \\
\hline 8 & 81 & 162 & 16 & 17 & 81 & 162 & 16 & 17 \\
\hline 9 & 100 & 200 & 18 & 19 & 100 & 200 & 18 & 19 \\
\hline 10 & 121 & 242 & 20 & 21 & 121 & 242 & 20 & 21 \\
\hline 11 & 144 & 288 & 22 & 23 & 144 & 288 & 22 & 23 \\
\hline 12 & 169 & 338 & 24 & 25 & 169 & 338 & 24 & 25 \\
\hline 13 & 196 & 392 & 26 & 27 & 196 & 392 & 26 & 27 \\
\hline 14 & 225 & 450 & 28 & 29 & 225 & 450 & 28 & 29 \\
\hline 15 & 256 & 512 & 30 & 31 & 256 & 512 & 30 & 31 \\
\hline 16 & 289 & 578 & 32 & 33 & 289 & 578 & 32 & 33 \\
\hline
\end{tabular}

\begin{tabular}{|c|c|c|c|c|c|c|c|c|}
\hline & \multicolumn{4}{|c|}{ DOUB_LEG-TCHEBY } & \multicolumn{4}{c|}{ TRI_LEG-TCHEBY } \\
\cline { 2 - 9 } Resolution & $\begin{array}{c}\text { Points } \\
\text { on } 2 \pi\end{array}$ & $\begin{array}{c}\text { Points } \\
\text { on } 4 \pi\end{array}$ & $\begin{array}{c}\text { Library } \\
\text { Order }\end{array}$ & $\begin{array}{c}\text { Order when } \\
\text { Error }<10 \text { E-6 }\end{array}$ & $\begin{array}{c}\text { Points } \\
\text { on } 2 \pi\end{array}$ & $\begin{array}{c}\text { Points } \\
\text { on 4 } \pi\end{array}$ & $\begin{array}{c}\text { Library } \\
\text { Order }\end{array}$ & $\begin{array}{c}\text { Order when } \\
\text { Error }<10 E-6\end{array}$ \\
\hline 1 & 8 & 16 & 2 & 3 & 4 & 8 & 2 & 3 \\
\hline 2 & 18 & 36 & 4 & 5 & 12 & 24 & 4 & 3 \\
\hline 3 & 32 & 64 & 6 & 7 & 24 & 48 & 6 & 3 \\
\hline 4 & 50 & 100 & 8 & 9 & 40 & 80 & 8 & 3 \\
\hline 5 & 72 & 144 & 10 & 11 & 60 & 120 & 10 & 3 \\
\hline 6 & 98 & 196 & 12 & 13 & 84 & 168 & 12 & 3 \\
\hline 7 & 128 & 256 & 14 & 15 & 112 & 224 & 14 & 3 \\
\hline 8 & 162 & 324 & 16 & 17 & 144 & 288 & 16 & 3 \\
\hline 9 & 200 & 400 & 18 & 19 & 180 & 360 & 18 & 3 \\
\hline 10 & 242 & 484 & 20 & 21 & 220 & 440 & 20 & 3 \\
\hline 11 & 288 & 576 & 22 & 23 & & & & \\
\hline 12 & 338 & 676 & 24 & 25 & & & & \\
\hline 13 & 392 & 784 & 26 & 27 & & & & \\
\hline 14 & 450 & 900 & 28 & 29 & & & & \\
\hline 15 & 512 & 1024 & 30 & 31 & & & & \\
\hline 16 & 578 & 1156 & 32 & 33 & & & & \\
\hline
\end{tabular}




\begin{tabular}{|c|c|c|c|c|c|c|c|c|c|}
\hline \multirow[b]{2}{*}{ Res. } & \multicolumn{4}{|c|}{ LEBEDEV-LAIKOV } & \multicolumn{5}{|c|}{ LEBEDEV-LAIKOV } \\
\hline & $\begin{array}{l}\text { Points } \\
\text { on } 2 \pi\end{array}$ & $\begin{array}{l}\text { Points } \\
\text { on } 4 \pi\end{array}$ & $\begin{array}{l}\text { Library } \\
\text { Order }\end{array}$ & $\begin{array}{l}\text { Order when } \\
\text { Error }<10 \mathrm{E}-6\end{array}$ & Res. & $\begin{array}{l}\text { Points } \\
\text { on } 2 \pi\end{array}$ & $\begin{array}{l}\text { Points } \\
\text { on } 4 \pi\end{array}$ & $\begin{array}{l}\text { Library } \\
\text { Order }\end{array}$ & $\begin{array}{l}\text { Order when } \\
\text { Error }<10 \mathrm{E}-6\end{array}$ \\
\hline 1 & 3 & 6 & 3 & 3 & 11 & 97 & 194 & 23 & 23 \\
\hline 2 & 7 & 14 & 5 & 5 & 12 & 115 & 230 & 25 & 25 \\
\hline 3 & 13 & 26 & 7 & 7 & 13 & 133 & 266 & 27 & 27 \\
\hline 4 & 19 & 38 & 9 & 9 & 14 & 151 & 302 & 29 & 29 \\
\hline 5 & 25 & 50 & 11 & 11 & 15 & 175 & 350 & 31 & 31 \\
\hline 6 & 37 & 74 & 13 & 13 & 16 & 217 & 434 & 35 & 35 \\
\hline 7 & 43 & 86 & 15 & 15 & 17 & 295 & 590 & 41 & 41 \\
\hline 8 & 55 & 110 & 17 & 17 & 18 & 385 & 770 & 47 & 47 \\
\hline 9 & 73 & 146 & 19 & 19 & 19 & 487 & 974 & 53 & 53 \\
\hline 10 & 85 & 170 & 21 & 21 & 20 & 601 & 1202 & 59 & 59 \\
\hline
\end{tabular}

\begin{tabular}{|c|c|c|c|c|c|c|c|c|}
\hline \multirow{2}{*}{ Resolution } & \multicolumn{4}{|c|}{ THURGOOD_TN } & \multicolumn{4}{c|}{ THURGOOD_LEASTSQ } \\
\cline { 2 - 9 } & $\begin{array}{c}\text { Points } \\
\text { on } 2 \pi\end{array}$ & $\begin{array}{c}\text { Points } \\
\text { on 4 } \pi\end{array}$ & $\begin{array}{c}\text { Library } \\
\text { Order }\end{array}$ & $\begin{array}{c}\text { Order when } \\
\text { Error }<10 \mathrm{E}-6\end{array}$ & $\begin{array}{c}\text { Points } \\
\text { on } 2 \pi\end{array}$ & $\begin{array}{c}\text { Points } \\
\text { on } 4 \pi\end{array}$ & $\begin{array}{c}\text { Library } \\
\text { Order }\end{array}$ & $\begin{array}{c}\text { Order when } \\
\text { Error }<10 \mathrm{E}-6\end{array}$ \\
\hline 1 & 4 & 8 & 3 & 3 & 4 & 8 & 3 & 3 \\
\hline 2 & 16 & 32 & 3 & 3 & 16 & 32 & 3 & 3 \\
\hline 3 & 36 & 72 & 3 & 3 & 36 & 72 & 3 & 3 \\
\hline 4 & 64 & 128 & 5 & 3 & 64 & 128 & 5 & 5 \\
\hline 5 & 100 & 200 & 7 & 3 & 100 & 200 & 7 & 7 \\
\hline 6 & 144 & 288 & 9 & 3 & 144 & 288 & 9 & 9 \\
\hline 7 & 196 & 392 & 11 & 3 & 196 & 392 & 11 & 11 \\
\hline 8 & 256 & 512 & 13 & 3 & 256 & 512 & 13 & 13 \\
\hline 9 & 324 & 648 & 15 & 3 & 324 & 648 & 15 & 15 \\
\hline 10 & 400 & 800 & 17 & 3 & 400 & 800 & 17 & 17 \\
\hline
\end{tabular}




\begin{tabular}{|c|c|c|c|c|c|c|c|c|}
\hline \multirow{2}{*}{ Resolution } & \multicolumn{4}{|c|}{ TEG_CORNER_EQWT } & \multicolumn{4}{c|}{ TEG_CORNER_LSQ } \\
\cline { 2 - 10 } & $\begin{array}{c}\text { Points } \\
\text { on } 2 \pi\end{array}$ & $\begin{array}{c}\text { Points } \\
\text { on 4 } \pi\end{array}$ & $\begin{array}{c}\text { Library } \\
\text { Order }\end{array}$ & $\begin{array}{c}\text { Order when } \\
\text { Error }<10 \text { E-6 }\end{array}$ & $\begin{array}{c}\text { Points } \\
\text { on } 2 \pi\end{array}$ & $\begin{array}{c}\text { Points } \\
\text { on } 4 \pi\end{array}$ & $\begin{array}{c}\text { Library } \\
\text { Order }\end{array}$ & $\begin{array}{c}\text { Order when } \\
\text { Error }<10 E-6\end{array}$ \\
\hline 1 & 6 & 12 & 3 & 5 & 6 & 12 & 3 & 5 \\
\hline 2 & 21 & 42 & 5 & 5 & 21 & 42 & 5 & 9 \\
\hline 3 & 46 & 92 & 9 & 5 & 46 & 92 & 9 & 11 \\
\hline 4 & 81 & 162 & 11 & 5 & 81 & 162 & 11 & 15 \\
\hline 5 & 126 & 252 & 15 & 5 & 126 & 252 & 15 & 17 \\
\hline 6 & 181 & 362 & 17 & 5 & 181 & 362 & 17 & 21 \\
\hline 7 & 246 & 492 & 21 & 5 & 246 & 492 & 21 & 23 \\
\hline 8 & 321 & 642 & 23 & 5 & 321 & 642 & 23 & 27 \\
\hline 9 & 406 & 812 & 27 & 5 & 406 & 812 & 27 & 29 \\
\hline 10 & 501 & 1002 & 31 & 5 & 501 & 1002 & 31 & 33 \\
\hline 11 & 606 & 1212 & 33 & 5 & 606 & 1212 & 33 & 9 \\
\hline 12 & 721 & 1442 & 37 & 5 & 721 & 1442 & 37 & 9 \\
\hline 13 & 846 & 1692 & 39 & 5 & 846 & 1692 & 39 & 5 \\
\hline 14 & 981 & 1962 & 43 & 5 & 981 & 1962 & 43 & 9 \\
\hline 15 & 1126 & 2252 & 45 & 5 & 1126 & 2252 & 45 & 49 \\
\hline
\end{tabular}

\begin{tabular}{|c|c|c|c|c|c|c|c|c|}
\hline \multirow{2}{*}{ Resolution } & \multicolumn{4}{|c|}{ TEG_CENTROID_EQW } & \multicolumn{4}{c|}{ TEG_CENTROID_LSQ } \\
\cline { 2 - 9 } & $\begin{array}{c}\text { Points } \\
\text { on } 2 \pi\end{array}$ & $\begin{array}{c}\text { Points } \\
\text { on 4 } \pi\end{array}$ & $\begin{array}{c}\text { Library } \\
\text { Order }\end{array}$ & $\begin{array}{c}\text { Order when } \\
\text { Error }<10 \text { E-6 }\end{array}$ & $\begin{array}{c}\text { Points } \\
\text { on } 2 \pi\end{array}$ & $\begin{array}{c}\text { Points } \\
\text { on } 4 \pi\end{array}$ & $\begin{array}{c}\text { Library } \\
\text { Order }\end{array}$ & $\begin{array}{c}\text { Order when } \\
\text { Error }<10 E-6\end{array}$ \\
\hline 1 & 10 & 20 & 3 & 5 & 10 & 20 & 3 & 5 \\
\hline 2 & 40 & 80 & 7 & 5 & 40 & 80 & 7 & 9 \\
\hline 3 & 90 & 180 & 11 & 5 & 90 & 180 & 11 & 5 \\
\hline 4 & 160 & 320 & 17 & 5 & 160 & 320 & 17 & 5 \\
\hline 5 & 250 & 500 & 21 & 5 & 250 & 500 & 21 & 5 \\
\hline 6 & 360 & 720 & 25 & 5 & 360 & 720 & 25 & 5 \\
\hline 7 & 490 & 980 & 29 & 5 & 490 & 980 & 29 & 5 \\
\hline 8 & 640 & 1280 & 35 & 5 & 640 & 1280 & 35 & 5 \\
\hline 9 & 810 & 1620 & 39 & 5 & 810 & 1620 & 39 & 5 \\
\hline 10 & 1000 & 2000 & 43 & 5 & 1000 & 2000 & 43 & 5 \\
\hline 11 & 1210 & 2420 & 47 & 5 & 1210 & 2420 & 47 & 5 \\
\hline 12 & 1440 & 2880 & 53 & 5 & 1440 & 2880 & 53 & 5 \\
\hline 13 & 1690 & 3380 & 57 & 5 & 1690 & 3380 & 57 & 5 \\
\hline 14 & 1960 & 3920 & 61 & 5 & 1960 & 3920 & 61 & 21 \\
\hline 15 & 2250 & 4500 & 65 & 5 & 2250 & 4500 & 65 & 11 \\
\hline
\end{tabular}




\begin{tabular}{|c|c|c|c|c|c|c|c|c|}
\hline & \multicolumn{4}{|c|}{ COBE_EVEN_EQWT } & \multicolumn{4}{c|}{ COBE_EVEN_LSQ } \\
\cline { 2 - 9 } Resolution & $\begin{array}{c}\text { Points } \\
\text { on } 2 \pi\end{array}$ & $\begin{array}{c}\text { Points } \\
\text { on } 4 \pi\end{array}$ & $\begin{array}{c}\text { Library } \\
\text { Order }\end{array}$ & $\begin{array}{c}\text { Order when } \\
\text { Error<10E-6 }\end{array}$ & $\begin{array}{c}\text { Points } \\
\text { on } 2 \pi\end{array}$ & $\begin{array}{c}\text { Points } \\
\text { on } 4 \pi\end{array}$ & $\begin{array}{c}\text { Library } \\
\text { Order }\end{array}$ & $\begin{array}{c}\text { Order when } \\
\text { Error }<10 E-6\end{array}$ \\
\hline 1 & 12 & 24 & 1 & 3 & 12 & 24 & 1 & 3 \\
\hline 2 & 48 & 96 & 7 & 3 & 48 & 96 & 7 & 3 \\
\hline 3 & 108 & 216 & 11 & 3 & 108 & 216 & 11 & 3 \\
\hline 4 & 192 & 384 & 17 & 3 & 192 & 384 & 17 & 3 \\
\hline 5 & 300 & 600 & 21 & 3 & 300 & 600 & 21 & 3 \\
\hline 6 & 432 & 864 & 27 & 3 & 432 & 864 & 27 & 3 \\
\hline 7 & 588 & 1176 & 31 & 3 & 588 & 1176 & 31 & 3 \\
\hline 8 & 768 & 1536 & 37 & 3 & 768 & 1536 & 37 & 3 \\
\hline 9 & 972 & 1944 & 41 & 3 & & & & \\
\hline 10 & 1200 & 2400 & 45 & 3 & & & & \\
\hline
\end{tabular}

\begin{tabular}{|c|c|c|c|c|c|c|c|c|}
\hline & \multicolumn{4}{|c|}{ COBE_ODD_EQWT } & \multicolumn{4}{c|}{ COBE_ODD_LSQ } \\
\cline { 2 - 9 } Resolution & $\begin{array}{c}\text { Points } \\
\text { on } 2 \pi\end{array}$ & $\begin{array}{c}\text { Points } \\
\text { on } 4 \pi\end{array}$ & $\begin{array}{c}\text { Library } \\
\text { Order }\end{array}$ & $\begin{array}{c}\text { Order when } \\
\text { Error<10E-6 }\end{array}$ & $\begin{array}{c}\text { Points } \\
\text { on } 2 \pi\end{array}$ & $\begin{array}{c}\text { Points } \\
\text { on } 4 \pi\end{array}$ & $\begin{array}{c}\text { Library } \\
\text { Order }\end{array}$ & $\begin{array}{c}\text { Order when } \\
\text { Error }<10 E-6\end{array}$ \\
\hline 1 & 3 & 6 & 1 & 3 & 3 & 6 & 1 & 3 \\
\hline 2 & 27 & 54 & 7 & 3 & 27 & 54 & 7 & 3 \\
\hline 3 & 75 & 150 & 11 & 3 & 75 & 150 & 11 & 3 \\
\hline 4 & 147 & 294 & 17 & 3 & 147 & 294 & 17 & 3 \\
\hline 5 & 243 & 486 & 21 & 3 & 243 & 486 & 21 & 3 \\
\hline 6 & 363 & 726 & 27 & 3 & 363 & 726 & 27 & 3 \\
\hline 7 & 507 & 1014 & 31 & 3 & 507 & 1014 & 31 & 3 \\
\hline 8 & 675 & 1350 & 37 & 3 & 675 & 1350 & 37 & 3 \\
\hline
\end{tabular}




\begin{tabular}{|c|c|c|c|c|c|c|c|c|}
\hline \multirow{2}{*}{ Resolution } & \multicolumn{4}{|c|}{ COBE_C_EVEN_EQWT } & \multicolumn{4}{c|}{ COBE_C_EVEN_LSQ } \\
\cline { 2 - 9 } & $\begin{array}{c}\text { Points } \\
\text { on } 2 \pi\end{array}$ & $\begin{array}{c}\text { Points } \\
\text { on } 4 \pi\end{array}$ & $\begin{array}{c}\text { Library } \\
\text { Order }\end{array}$ & $\begin{array}{c}\text { Order when } \\
\text { Error }<10 \mathrm{E}-6\end{array}$ & $\begin{array}{c}\text { Points } \\
\text { on } 2 \pi\end{array}$ & $\begin{array}{c}\text { Points } \\
\text { on } 4 \pi\end{array}$ & $\begin{array}{c}\text { Library } \\
\text { Order }\end{array}$ & $\begin{array}{c}\text { Order when } \\
\text { Error }<10 E-6\end{array}$ \\
\hline 1 & 4 & 8 & 2 & 3 & 4 & 8 & 2 & 3 \\
\hline 2 & 28 & 56 & 5 & 3 & 28 & 56 & 5 & 7 \\
\hline 3 & 76 & 152 & 12 & 3 & 76 & 152 & 12 & 3 \\
\hline 4 & 148 & 296 & 16 & 3 & 148 & 296 & 16 & 17 \\
\hline 5 & 244 & 488 & 22 & 3 & 244 & 488 & 22 & 3 \\
\hline 6 & 364 & 728 & 26 & 3 & 364 & 728 & 26 & 27 \\
\hline 7 & 508 & 1016 & 32 & 3 & 508 & 1016 & 32 & 3 \\
\hline 8 & 676 & 1352 & 36 & 3 & 676 & 1352 & 36 & 3 \\
\hline 9 & 868 & 1736 & 40 & 3 & 868 & 1736 & 40 & 41 \\
\hline 10 & 1084 & 2168 & 44 & 3 & 1084 & 2168 & 44 & 45 \\
\hline
\end{tabular}

\begin{tabular}{|c|c|c|c|c|c|c|c|c|}
\hline & \multicolumn{4}{|c|}{ COBE_C_ODD_EQWT } & \multicolumn{4}{c|}{ COBE_C_ODD_LSQ } \\
\cline { 2 - 10 } Resolution & $\begin{array}{c}\text { Points } \\
\text { on } 2 \pi\end{array}$ & $\begin{array}{c}\text { Points } \\
\text { on } 4 \pi\end{array}$ & $\begin{array}{c}\text { Library } \\
\text { Order }\end{array}$ & $\begin{array}{c}\text { Order when } \\
\text { Error }<10 \mathrm{E}-6\end{array}$ & $\begin{array}{c}\text { Points } \\
\text { on } 2 \pi\end{array}$ & $\begin{array}{c}\text { Points } \\
\text { on } 4 \pi\end{array}$ & $\begin{array}{c}\text { Library } \\
\text { Order }\end{array}$ & $\begin{array}{c}\text { Order when } \\
\text { Error }<10 E-6\end{array}$ \\
\hline 1 & 13 & 26 & 5 & 3 & 13 & 26 & 5 & 7 \\
\hline 2 & 49 & 98 & 10 & 3 & 49 & 98 & 10 & 11 \\
\hline 3 & 109 & 218 & 16 & 3 & 109 & 218 & 16 & 17 \\
\hline 4 & 193 & 386 & 24 & 3 & 193 & 386 & 24 & 3 \\
\hline 5 & 301 & 602 & 24 & 3 & 301 & 602 & 24 & 25 \\
\hline 6 & 433 & 866 & 30 & 3 & 433 & 866 & 30 & 31 \\
\hline 7 & 589 & 1178 & 36 & 3 & 589 & 1178 & 36 & 3 \\
\hline 8 & 769 & 1538 & 40 & 3 & 769 & 1538 & 40 & 41 \\
\hline 9 & 973 & 1946 & 46 & 3 & 973 & 1946 & 46 & 3 \\
\hline 10 & 1201 & 2402 & 52 & 3 & 1201 & 2402 & 52 & 3 \\
\hline
\end{tabular}


The number of directions associated with the THETA_RESOLUTION is found to be $\theta+1$ directions on $4 \pi$ and $\frac{1}{2}(\theta+1)$ directions on $2 \pi$. The number of directions associated with PHI_RESOLUTION is indicated in the figure in the next page noting that for $2 \pi$, only one of the directions that travels in the $\mathrm{Y}$ direction will become part of the cubature for one when there is one direction traveling in the $x-y$ plane associated with THETA_RESOLUTION. This usually leads to prime numbers for the section of angles and thus we strongly suggest you stick to using odd resolution numbers. Also note the $\mathrm{x}-\mathrm{y}$ symmetries typically imposed for reactor geometries can be exactly handled by selecting specific resolutions of the PHI_RESOLUTION as indicated below. 

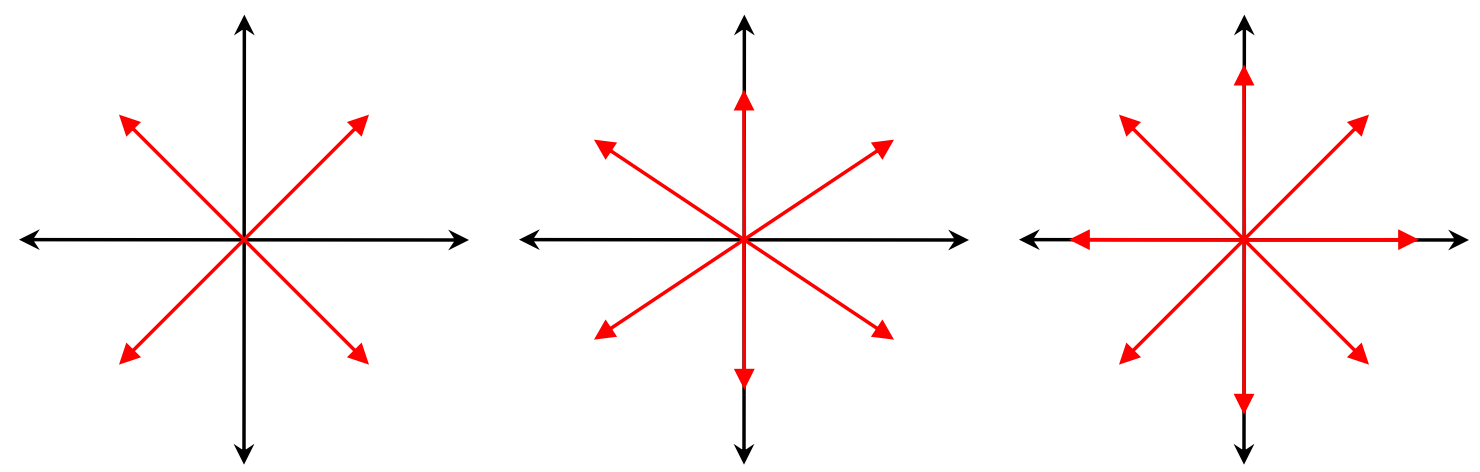

\begin{tabular}{c} 
Res. 1 \\
$\delta=\frac{\pi}{2}=90^{\circ}$ \\
\hline
\end{tabular}

\begin{tabular}{c} 
Res. 2 \\
$\delta=\frac{\pi}{3}=60^{\circ}$ \\
\hline
\end{tabular}

Res. 3

$$
\delta=\frac{\pi}{4}=45^{\circ}
$$
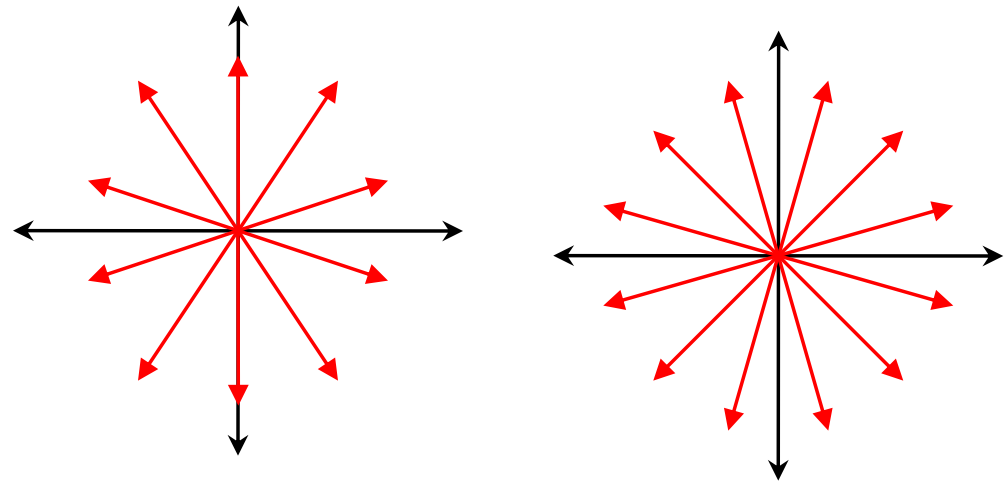

$$
\begin{gathered}
\text { Res. } 4 \\
\delta=\frac{\pi}{5}=36^{\circ}
\end{gathered}
$$

Res. 5

$$
\delta=\frac{\pi}{6}=30^{\circ}
$$

\section{General Tchebychev Relation}

$$
\delta=\frac{\pi}{(R+1)} \quad \text { Points }=2(R+1) \quad \phi=\frac{\delta}{2}+(i-1) \delta \quad i \in 1 \text {, Points }
$$

Resolutions with 45 degree symmetry $=1,3,5,7,9, \ldots$ odd

Resolutions with 30 or 60 degree symmetry $=2,5,8,11,14,17,20,23$ 


\section{Appendix B. Example PROTEUS-SN Driver Input File: “sn2nd.inp"}

The following is an example of the driver input file for PROTEUS-SN, taken from bench05. This problem uses Legendre-Tchebyshev cubature of order L3T9. It uses Tchebychev acceleration and the SSOR preconditioner. The maximum number of outer iterations is set to 100 and the upscatter iterations are limited to 5. Note that this driver input files points to the other required input files using the SOURCEFILE_MESH, SOURCEFILE_MATERIAL, SOURCEFILE_XS keywords.

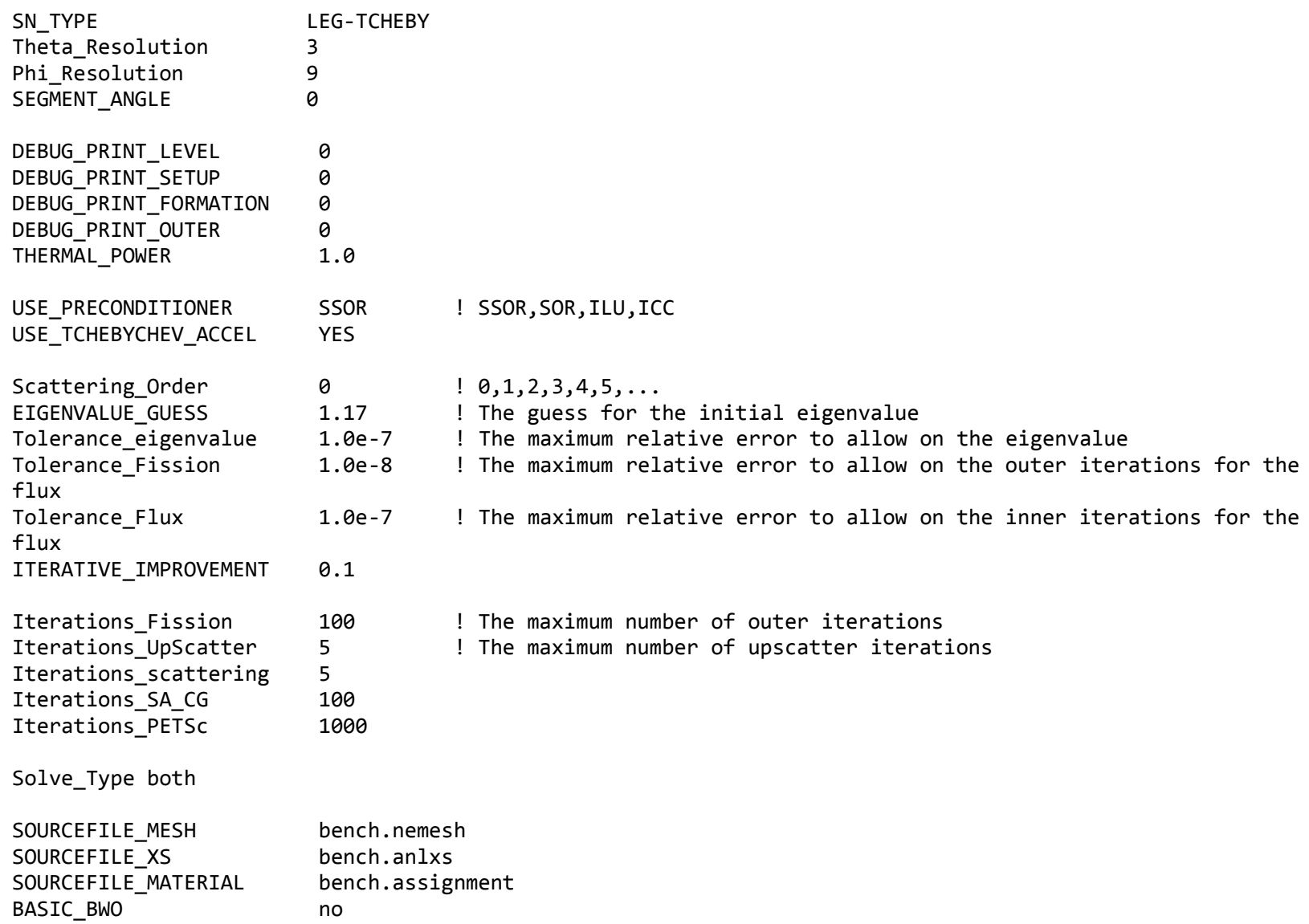




\section{Appendix C. Cross Section File Format for PROTEUS-SN: "anlxs"}

This section specifies the PROTEUS-SN "anlxs" cross section file format. An example input file is also provided.

\section{C.1 File format description for "anlxs" cross section file}

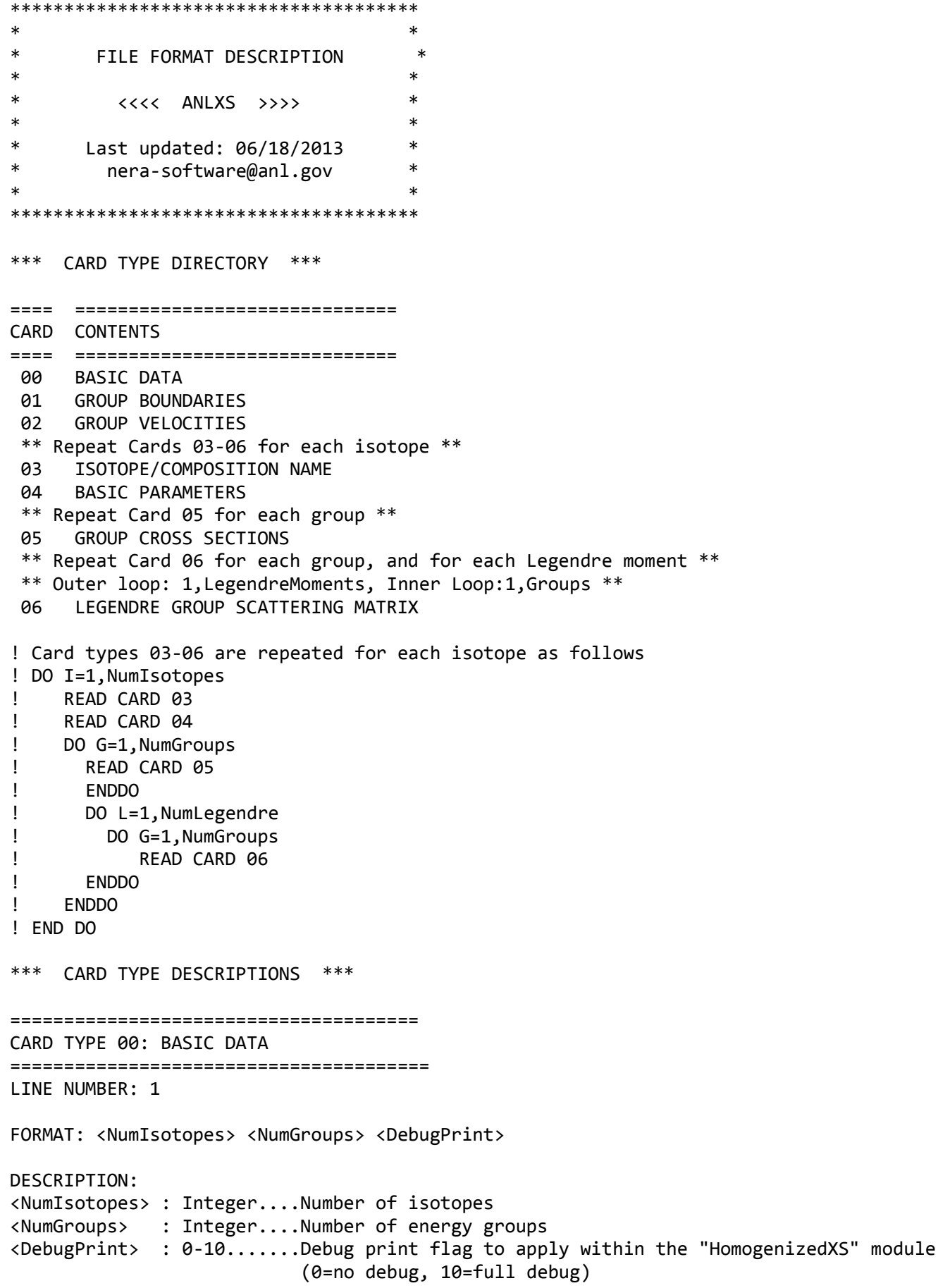




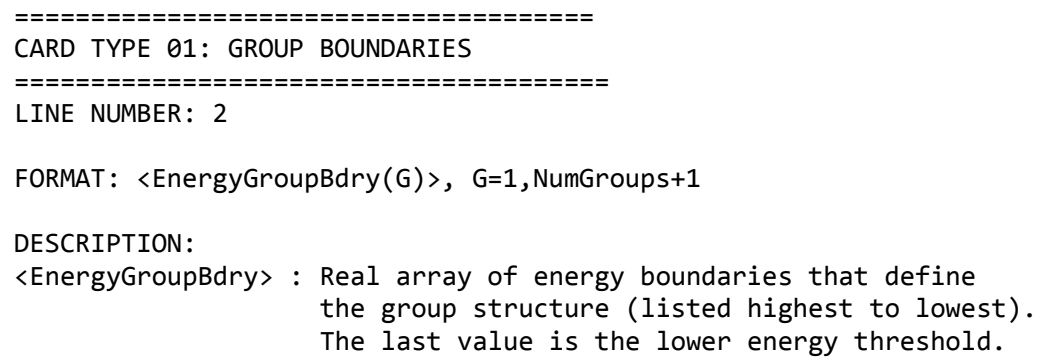




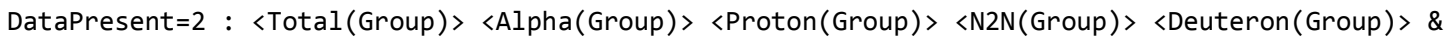
$\langle\operatorname{Tritium}($ Group) $\rangle\langle$ Fission(Group) $\rangle\langle$ NuFission(Group) $\rangle\langle$ Chi(Group) $\rangle$

DESCRIPTION:

The multigroup cross section data for the given isotope and group are given according to the above formats.

The types of cross sections are defined here:

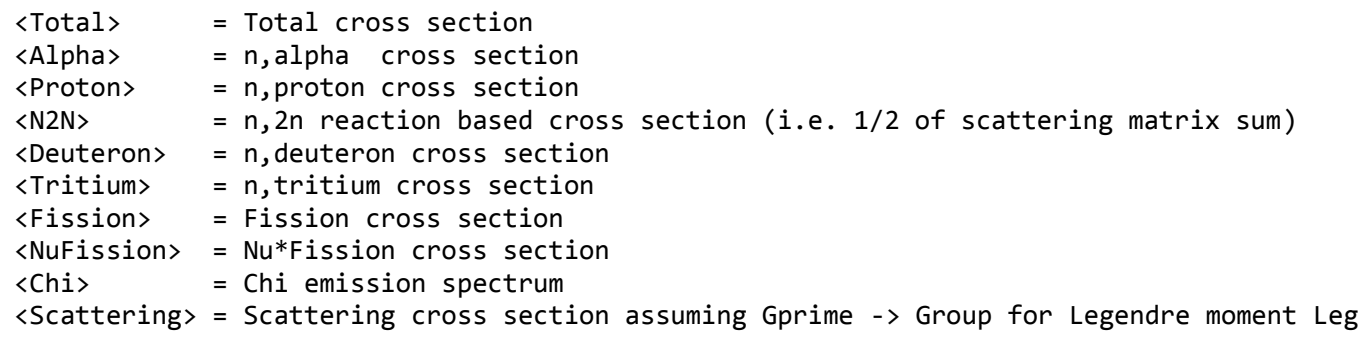

\section{C.2 Example "anlxs" cross section file (taken from bench02 directory)}

320

$1.0000 \mathrm{E}+07 \quad 6.82560 \mathrm{E}-01 \quad 1.00000 \mathrm{E}-05$

$1.00000 \mathrm{E}+051.00000 \mathrm{E}-01$

Fuel

$\begin{array}{llllll}1 & 1 & 1.0 & 0.0 & 0.602214179\end{array}$

$\begin{array}{llll}0.22 & 0.003 & 0.006 & 1.0\end{array}$

$\begin{array}{llll}0.8 & 0.05 & 0.1 & 0.0\end{array}$

0.1930 .017

$0.0 \quad 0.73$

clad

$\begin{array}{llllll}1 & 0 & 1.0 & 0.0 & 0.602214179\end{array}$

0.53

0.94

$0.526 \quad 0.001$

$0.0 \quad 0.83$

Moderato

$\begin{array}{lllll}1 & 0 & 1.0 & 0.0 & 0.602214179\end{array}$

0.701

2.00

0.650 .05

$0.0 \quad 1.97$ 


\section{Appendix D. Mesh File Format Specification for PROTEUS-SN: "nemesh"}

This section specifies the PROTEUS-SN "nemesh" mesh file format. An example "nemesh" input file is also provided.

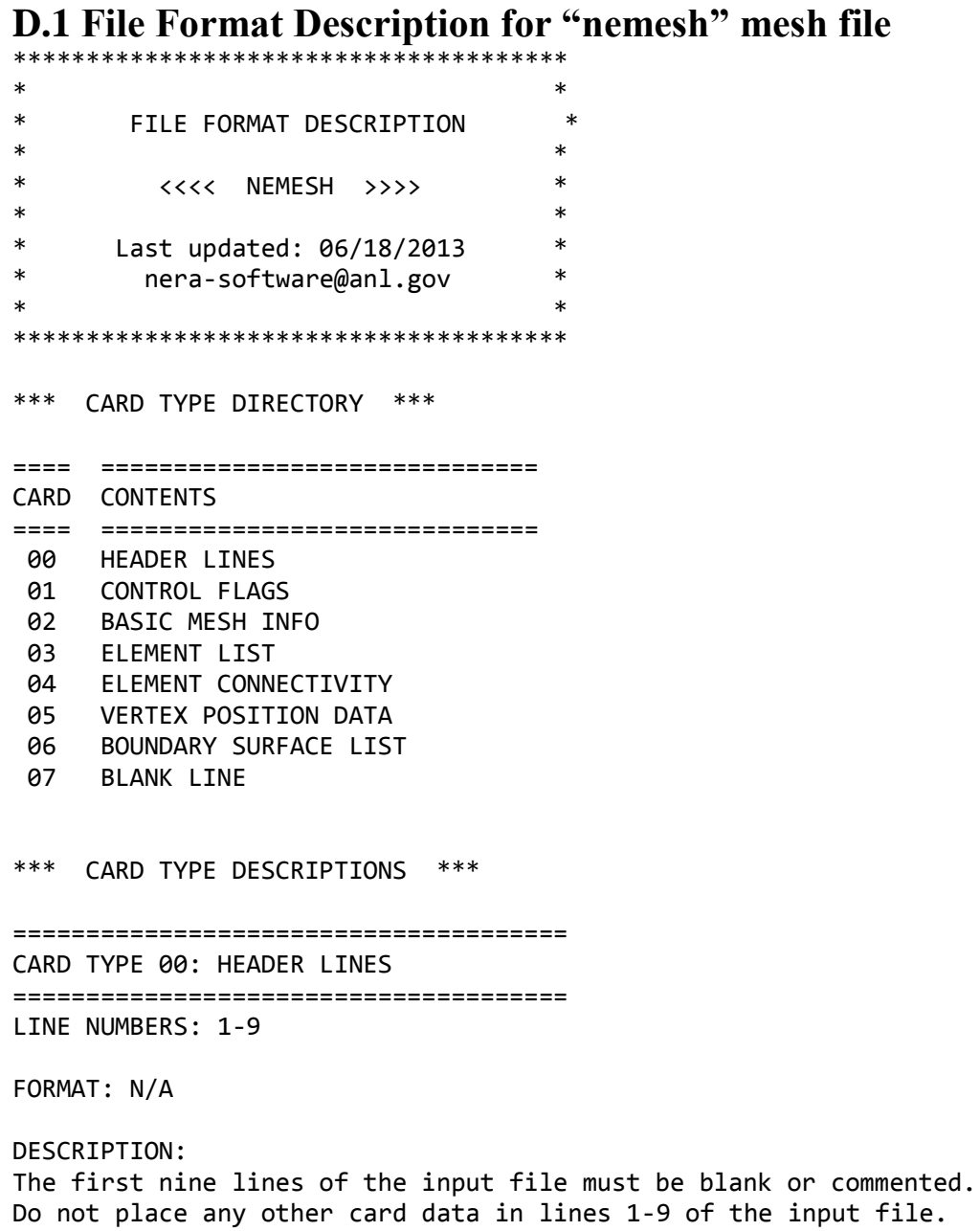


DESCRIPTION :

$\langle$ NumElements $>$

: Integer.... Number of elements in the mesh

$\langle$ NumNodes>

: Integer.... Number of nodes (vertices) in the mesh

<unused integer>

: $0 \ldots \ldots \ldots$ (reserved for future use)

<NumBoundarySurfaces>: Integer.... Number of boundary surfaces in the mesh

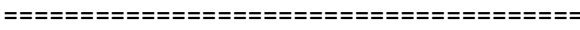

CARD TYPE 03: ELEMENT LIST

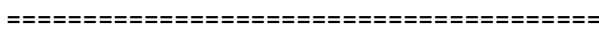

LINE NUMBERS: $12+$, with a total of NumElements lines

FORMAT: \{ [Optional Index] 〈ElementType〉<MaterialBlock〉 \}; repeat for each element specified in <NumElements>

DESCRIPTION :

[Optional Index] : Integer.... Optional index of the element

$\langle$ ElementType> : Integer from the following list

1...1-D Bar Linear

2....1-D Bar Quadratic

5...2-D Triangular Linear

6....2-D Triangular Quadratic

10....2-D Quadrilateral Linear

11....2-D Quadrilateral Quadratic

15....3-D Tetrahedron Linear

16....3-D Tetrahedron Quadratic

20....3-D Tri. Prismatic Linear

21....3-D Tri. Prismatic Quadratic

25....3-D Hexadron Linear

26....3-D Hexadron Quadratic

Lagrangian ( $31=$ linear, $39=9$ th order)

41-49....2-D Triangular

Lagrangian ( $41=$ linear, $49=9$ th order $)$

$51-59 . . .2$-D Quadrilateral Lagrangian (51 = linear, $59=9$ th order)

61-69....3-D Tetrahedron Lagrangian $(61=$ linear, $69=9$ th order $)$

71-79....3-D Tri. Prismatic Lagrangian (71 = linear, $79=9$ th order)

81-89....3-D Hexadron Lagrangian ( 81 = linear, $89=9$ th order $)$

91-99....2-D Quadrilateral NonConforming (Lagrangian setup)

101-109....3-D Hexadron NonConforming (Lagrangian setup)

<MaterialBlock> : Integer.... Material block for this element

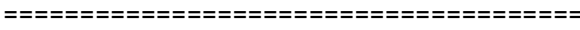

CARD TYPE 04: ELEMENT CONNECTIVITY

$===============================$

LINE NUMBERS: Immediately following last CARD TYPE 03, with a total of NumElements lines

FORMAT: \{ [Optional Index] <ConnectivityArray> \}; repeat for each element specified in 〈NumElements>

DESCRIPTION :

[Optional Index] : Integer.... Optional index of the element

<ConnectivityArray> : Array of integers read as CONNECTIVITY(J), J=1, ELEMENTVERTICES

This array lists the indexed vertices which define the element. ELEMENTVERTICES is determined automatically from ElementType.

For example ElementType 1 (1-D Bar Linear) has 2 vertices.

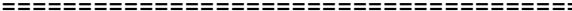

CARD TYPE 05: VERTEX POSITION DATA

$===================================$

LINE NUMBERS: Immediately following last CARD TYPE 04, with a total of NumNodes lines 
FORMAT: $\{$ [Optional Index] $\langle\mathrm{X}\rangle[\mathrm{Y}][\mathrm{Z}]\}$;

repeat for each node specified in 〈NumNodes>

DESCRIPTION :

[Optional Index] : Integer.... Optional index of the node

$\langle\mathrm{X}\rangle \quad$ : Real....X-value of this node (always present)

[Y] : Real....Y-value of this node (present only for 2D and 3D elements)

: Real....Z-value of this node (present only for 3D elements)

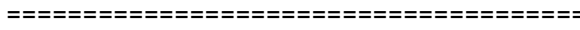

CARD TYPE 06: BOUNDARY SURFACE LIST

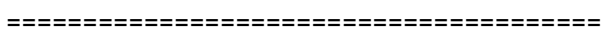

LINE NUMBERS: Immediately following last CARD TYPE 05, with a total of NumBoundarySurfaces lines

FORMAT: \{ [Optional Index] 〈ElementIndex> <ReferenceSurface〉<BoundaryConditionFlag〉 \}; repeat for each node specified in 〈NumNodes>

DESCRIPTION :

[Optional Index] : Integer.... Optional index of the boundary surface

$<$ ElementIndex> $\quad$ : Integer..... Index of the element with the boundary surface

<ReferenceSurface> : Integer....Reference element surface number corresponding to the boundary surface

〈BoundaryConditionFlag〉: $1 . .$. Reflected

other.....Void

\section{CARD TYPE 07: BLANK LINE}

$===================================$

LINE NUMBER: Last line in the file

FORMAT: blank line

DESCRIPTION:

A blank line must be placed at the end of the line in order to avoid "end of file" error upon reading.

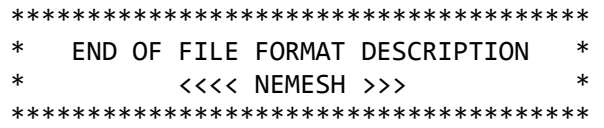

\section{D.2 Example "nemesh" file (taken from bench05)}

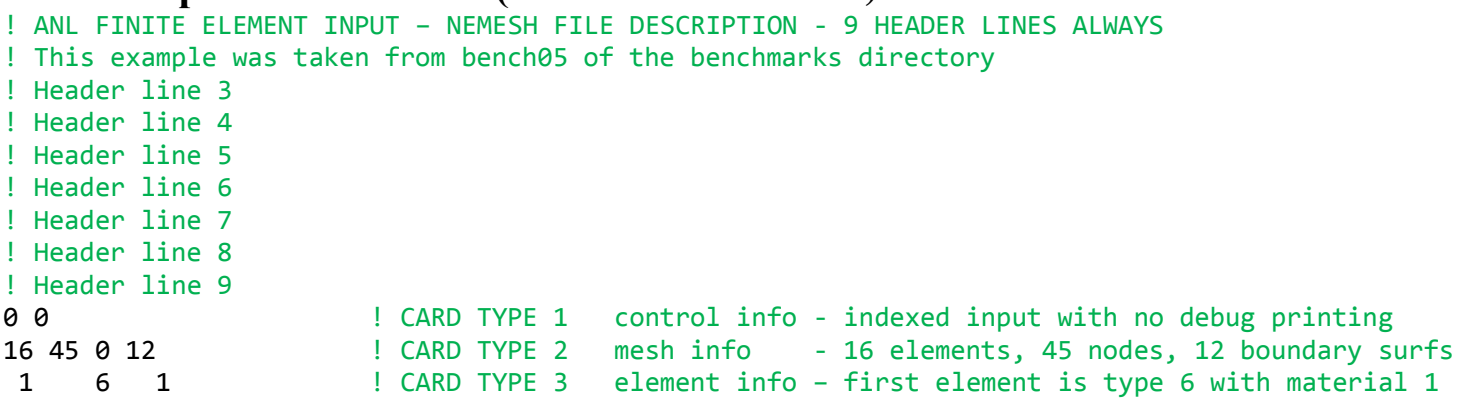


PROTEUS-SN User Manual

July 31, 2015

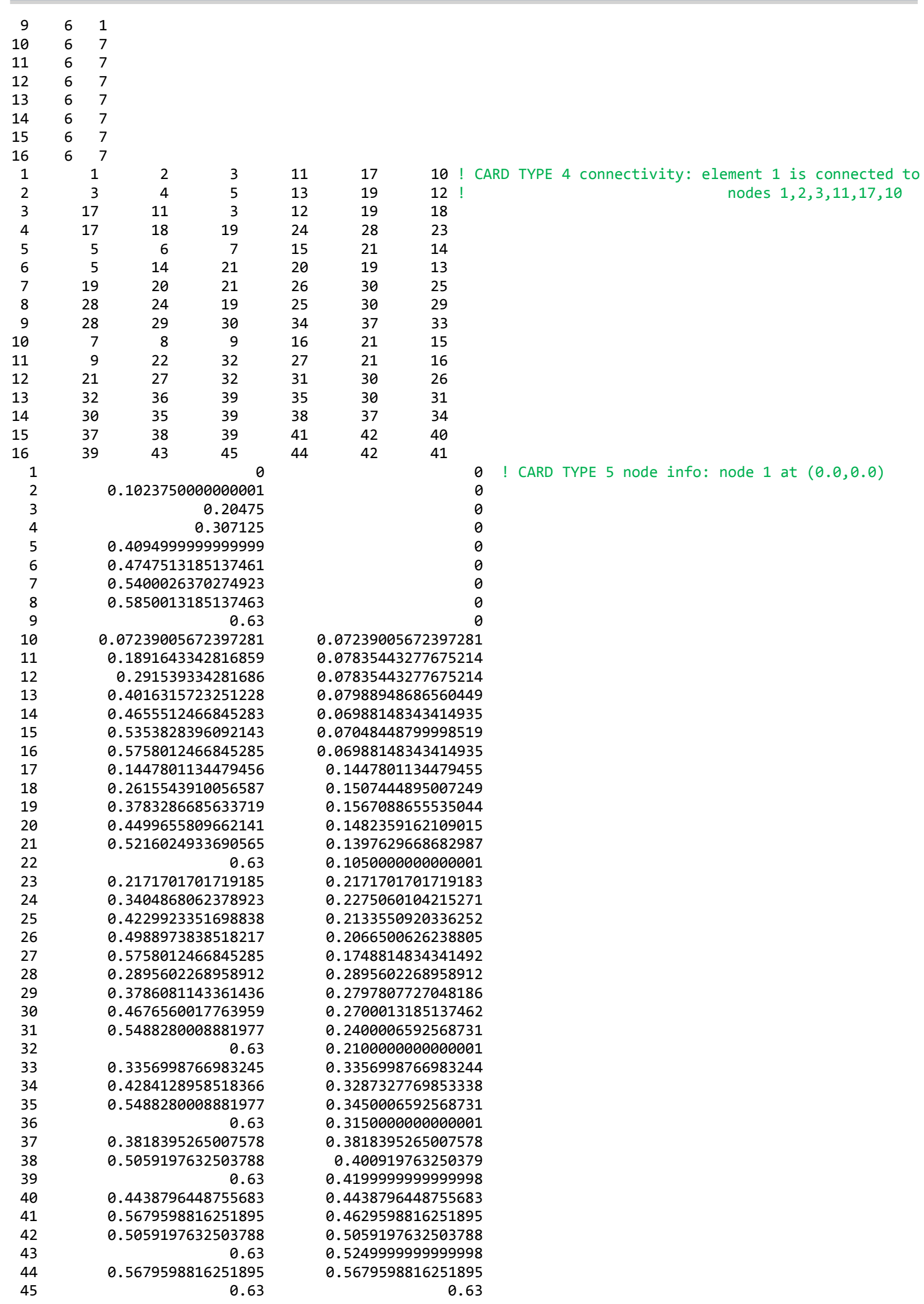


$\begin{array}{llll}1 & 1 & 1 & 1\end{array}$ $\begin{array}{lll}1 & 3 & 1\end{array}$

$\begin{array}{lll}2 & 1 & 1\end{array}$

$\begin{array}{lll}4 & 3 & 1\end{array}$

$\begin{array}{lll}5 & 1 & 1\end{array}$

$\begin{array}{llll}9 & 3 & 1\end{array}$

$\begin{array}{llll}7 & 10 & 1 & 1\end{array}$

$8 \quad 1111$

$\begin{array}{llll}9 & 13 & 1 & 1\end{array}$

$\begin{array}{llll}10 & 15 & 3 & 1\end{array}$

$\begin{array}{llll}11 & 16 & 1 & 1\end{array}$

$\begin{array}{llll}12 & 16 & 2 & 1\end{array}$
! CARD TYPE 6 boundary data: the first boundary surface is on ele 1 , ref. surf 1 , refl bc ! MAKE CERTAIN THERE IS AN EXTRA BLANK LINE AFTER ALL OF THE INPUT TO PREVENT EOF ERROR 


\section{Appendix E. Mesh File Format Specification for PROTEUS-SN: "ascii"}

This section specifies the PROTEUS-SN "ascii" mesh file format. An example input file is also provided.

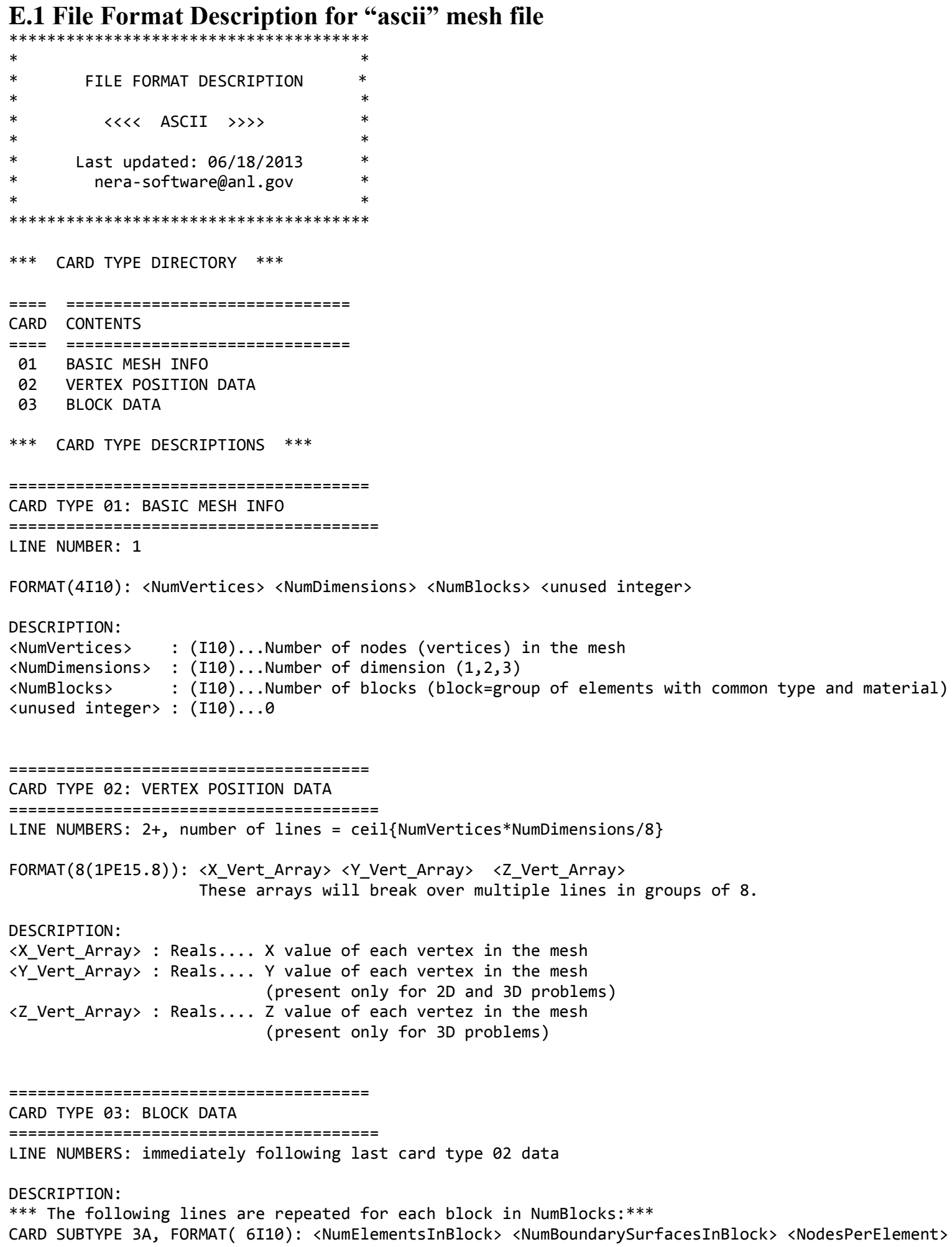




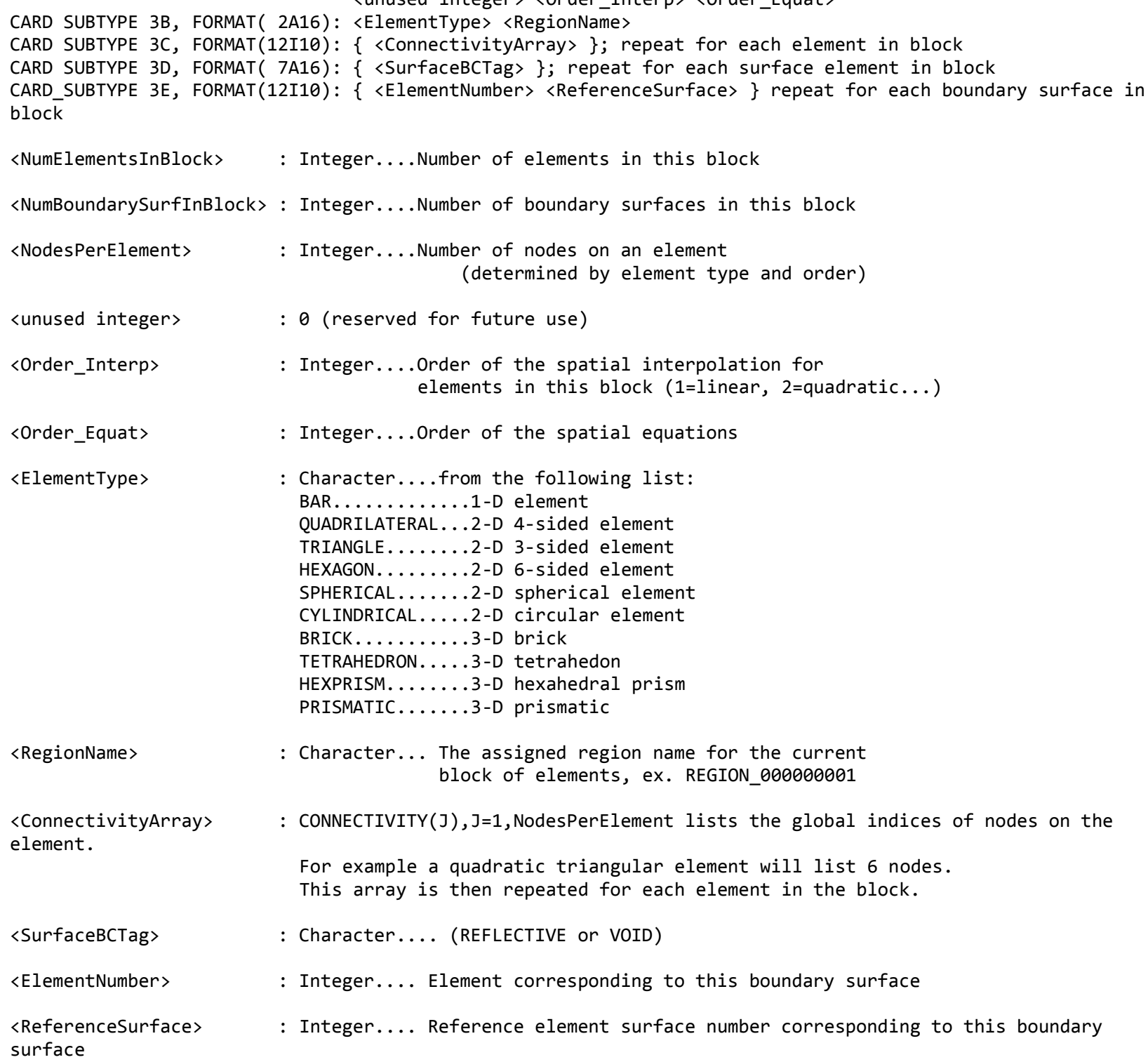




\section{E.2 Example "ascii" mesh file (converted from bench05 nemesh file)}

45

2

0

0.00000000E+00 1.02375000E-01 2.04750000E-01 3.07125000E-01 4.09500000E-01 4.74751319E-01 5.40002637E-01 5.85001319E-01 6.30000000E-01 7.23900567E-02 1.89164334E-01 2.91539334E-01 4.01631572E-01 4.65551247E-01 5.35382840E-01 5.75801247E-01 $\begin{array}{lllllllll}1.44780113 E-01 & 2.61554391 E-01 & 3.78328669 E-01 & 4.49965581 E-01 & 5.21602493 E-01 & 6.30000000 E-01 & 2.17170170 E-01 & 3.40486806 E-01\end{array}$ 4.22992335E-01 4.98897384E-01 5.75801247E-01 2.89560227E-01 3.78608114E-01 4.67656002E-01 5.48828001E-01 6.30000000E-01 3.35699877E-01 4.28412896E-01 5.48828001E-01 6.30000000E-01 3.81839527E-01 5.05919763E-01 6.30000000E-01 4.43879645E-01 $\begin{array}{lllllllll}5.67959882 E-01 & 5.05919763 E-01 & 6.30000000 E-01 & 5.67959882 E-01 & 6.30000000 E-01 & 0.00000000 E+00 & 0.00000000 E+00 & 0.00000000 E+00\end{array}$ $0.00000000 \mathrm{E}+00 \quad 0.00000000 \mathrm{E}+00 \quad 0.00000000 \mathrm{E}+00 \quad 0.00000000 \mathrm{E}+00 \quad 0.00000000 \mathrm{E}+00 \quad 0.00000000 \mathrm{E}+00 \quad 7.23900567 \mathrm{E}-02 \quad 7.83544328 \mathrm{E}-02$ 7.83544328E-02 7.98894869E-02 6.98814834E-02 7.04844880E-02 6.98814834E-02 $1.44780113 \mathrm{E}-01$ 1.50744490E-01 $1.56708866 \mathrm{E}-01$ $\begin{array}{llllllllllll}1.48235916 E-01 & 1.39762967 E-01 & 1.05000000 E-01 & 2.17170170 E-01 & 2.27506010 E-01 & 2.13355092 E-01 & 2.06650063 E-01 & 1.74881483 E-01\end{array}$ $\begin{array}{lllllllll}2.89560227 E-01 & 2.79780773 E-01 & 2.70001319 E-01 & 2.40000659 E-01 & 2.10000000 E-01 & 3.35699877 E-01 & 3.28732777 E-01 & 3.45000659 E-01\end{array}$ 3.15000000E-01 3.81839527E-01 4.00919763E-01 4.20000000E-01 4.43879645E-01 4.62959882E-01 5.05919763E-01 5.25000000E-01 $5.67959882 \mathrm{E}-01 \quad 6.30000000 \mathrm{E}-01$

\begin{tabular}{|c|c|c|c|c|c|}
\hline 9 & 6 & 6 & 0 & 2 & \\
\hline TRIANGLE & \multicolumn{5}{|c|}{ REGION_000000001 } \\
\hline 1 & 2 & 3 & 11 & 17 & \\
\hline 17 & 11 & 3 & 12 & 19 & \\
\hline 5 & 6 & 7 & 15 & 21 & \\
\hline 19 & 20 & 21 & 26 & 30 & \\
\hline 28 & 29 & 30 & 34 & 37 & \\
\hline REFLECTIVE & REFLECTIVE & & REFLECTIVE & $\mathrm{RE}$ & \\
\hline 1 & 1 & 1 & 3 & 2 & \\
\hline 7 & 6 & 6 & 0 & 2 & \\
\hline TRIANGLE & \multicolumn{5}{|c|}{ REGION_000000007 } \\
\hline 7 & 8 & 9 & 16 & 21 & \\
\hline 21 & 27 & 32 & 31 & 30 & \\
\hline 30 & 35 & 39 & 38 & 37 & \\
\hline 39 & 43 & 45 & 44 & 42 & \\
\hline REFLECTIVE & REFLECTIVE & & REFLECTIVE & & \\
\hline
\end{tabular}

2

10

14

25

33

1

REFLECTIVE

4

9

32
37

REFLECTIVE

6
1 REFLECT

4
18
14
24

5
19
21

REFLECTIVE$$
3
$$

22

36
38

R
REFLECTIVE
13

24

20

25

1

9

$\begin{array}{lll}1 & 2 & 1\end{array}$
4

\author{
32 \\ 39 \\ 7
}

31

27

41

$\begin{array}{ll}21 & 16 \\ 30 & 31 \\ 42 & 40\end{array}$

1
12

13

29 


\section{Appendix F. Example PROTEUS-SN Text Output File}

The following is an example output file taken from bench05, running in parallel with 4 processors.

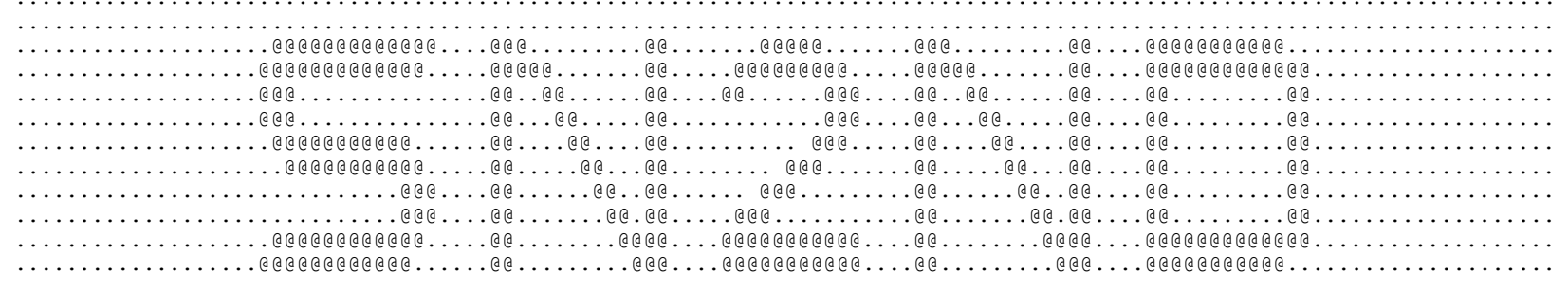

Argonne National Laboratory Neutronics Package (UNIC)

A steady-state solver of the multigroup neutron transport equation

Geometry inputs include Cartesian 1-D, 2-D, 3-D unstructured FE meshes

\begin{tabular}{|c|c|c|}
\hline & & \\
\hline $\begin{array}{l}\text { Dinesh Kaushik } \\
\text { Yunzhao Li } \\
\text { Abel Marin-Lafleche } \\
\text { Cristian Rabiti } \\
\text { Micheal A. Smith } \\
\text { Allan Wollaber }\end{array}$ & $\begin{array}{l}\text { KAUST/ANL } \\
\text { Argonne National Lab } \\
\text { Argonne National Lab } \\
\text { Idaho National Lab } \\
\text { Argonne National Lab } \\
\text { Los Alamos National Lab }\end{array}$ & $\begin{array}{l}\text { Dinesh.Kaushik@KAUST.EDU.SA } \\
\text { yli@anl.gov } \\
\text { amarinlafleche@anl.gov } \\
\text { masmith@anl.gov }\end{array}$ \\
\hline Component Library & de contributors and Respo & nsible Parties \\
\hline $\begin{array}{l}\text { PN2ND } \\
\text { SN2ND } \\
\text { MOCFE } \\
\text { NODAL }\end{array}$ & $\begin{array}{l}\text { cheal A. Smith, Dinesh Ka } \\
\text { cheal A. Smith \& Allan Wo } \\
\text { cheal A. Smith, Abel Mari } \\
\text { cheal A. Smith, Yunzhao I }\end{array}$ & $\begin{array}{l}\text { llaber, Dinesh Kaushik } \\
\text { n-Lafleche, Christian Rabit } \\
\text { i, Elmer Lewis }\end{array}$ \\
\hline
\end{tabular}

[SN2ND] $\ldots$ Successfuily imported the UNIC input (ail errors reported above)

SN2ND] ...Successfully converted bench.anlxs $\quad$ to ISOTXS formatted Converted ANLXS TO.ISOTXS...

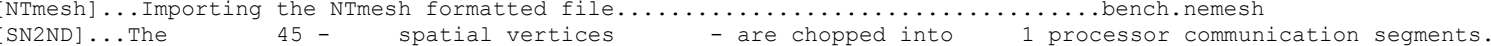

SN2ND]...The $\quad 20-\quad$ angular moments $\quad$ - are chopped into 4 processor communication segments...

SN2ND]...The $\quad 7$ - energy groups - are chopped into 1 processor communication segments...

[SN2ND]...Successfully imported the ASSIGNMENT input (all errors reported above).....

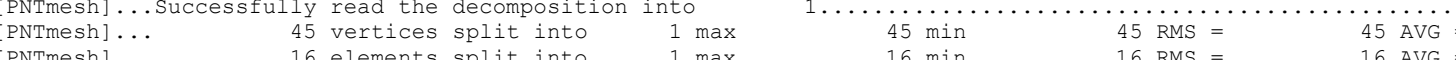

[PNTmesh]... 16 elements split into $19 \mathrm{max} \quad 16 \mathrm{~min} \quad 16$ RMS $=16$ AVG

[SN2ND] ...Neutron transport spatial mesh data was imported and the alias names were applied....

.

SN2ND.... BC matrices...................

SN2ND] . . obtaining the preconditioner matrices for the second order discrete ordinates method... 
[SN2ND] ... Debug print setting for iteration history....

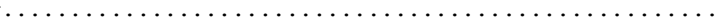

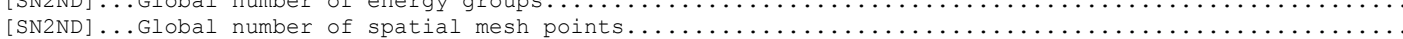

[SN2ND]... Corresponding number of finite elements. .

[SN2ND] ... Number of spatial dimensions....

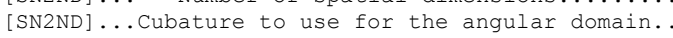

Global angular expansion order....

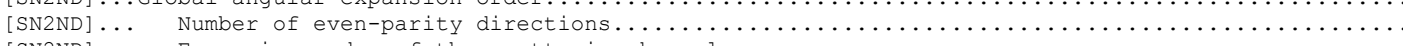

[SN2ND] $\ldots$ Expansion order of the scattering kerne $\ldots . .$.

[SN2ND] $\ldots$. Even-parity spherical harmonics. . .

[SN2ND]....Global space-angle degrees of freedom......... 
PROTEUS-SN User Manual

July 31, 2015

[SN2ND]

[SN2ND].

[SN2ND] ..

[SN2ND]

SN2ND]..

[SN2ND]

[SN2ND]

[SN2ND].

[SN2ND]...

SN2ND]

[SN2ND]

SN2ND]

$[$ [SN2ND]

[SN2ND]

SN2ND

[SN2ND]

[SN2ND

$[$ [SN2ND]

SN2ND]

SN2ND

SN2ND]

SN2ND]

[SN2ND]

[SN2ND]

SN2ND]

SN2ND] .

[SN2ND]

[SN2ND] ...

[SN2ND]

SN2ND]

SN2ND] ...

[SN2ND] .

SN2ND]...

[SN2ND]...

[SN2ND]..

[SN2ND]

[SN2ND]

SN2ND].

$=$ SN2ND

$=S N 2 N D$
$=S N 2 N D$

$=S N 2 N D$
$=S N 2 N D$

$=S N 2 N D$

$=S N 2 \mathrm{ND}$

-SN2ND

$=$ SN2N

$=$ SN2ND

$=$ SN2ND

$=$ SN2ND

$=\mathrm{SN} 2 \mathrm{~N}$

$=\mathrm{SN} 2 \mathrm{ND}$

$=\mathrm{SN} 2 \mathrm{ND}$

$=S N 2 \mathrm{ND}$

ANL/NE-14/6 (Rev 2.0$)$

.

. bebug print setting for iteration histor...

lobal number of energy groups...

Corresponding number of finite elements...

dimensions...

to use for the angular domain....

Number of even-parity direction

enton order of the scattering kernel...

Even-parity spherical harmonics.

Global space-angle degrees of freedom.

ince type information.

Is a fixed source present...

Is a up/down scattering present.

ive

terative improvement scaling factor....

Use Tchebychev acceleration........

rargeted error on the fission source.

Targeted error on the MGS flux solution.

Maximum fission source iterations....

cattering iteration control variables....

se synthetic diffusion acceleration. ...

Maximum space-angle CG iterations....

Maximum PETSC preconditioner iterations...

.

Vector memory usage.

Memory usage for A matrices.

Memory usage for PC matrices...........

Wery usage for fission sourc...

cratch memory for power iteration.

Total tracked memory usage (overhea...

tolal reported memory usage. ...

the preceding $\mathrm{Sn}$ system of equation

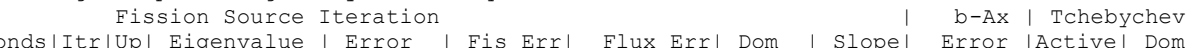

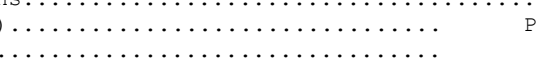

PEAK

$0.001900 \ldots$.

$0.022003 \ldots$

0.001572

0.076134 .

0.014420

$0.005127 \ldots$

$0.041199 \ldots$

0.171005. $0.1|001| 01|1.22808 \mathrm{E}+00| 5.0 \mathrm{E}-02|5.0 \mathrm{E}-02| \mathrm{T} \quad 8.5 \mathrm{E}-03|0.000| 0.000|4.5 \mathrm{E}-02| \mathrm{T} \quad 0|0.050|$

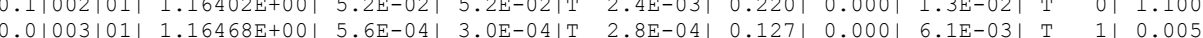

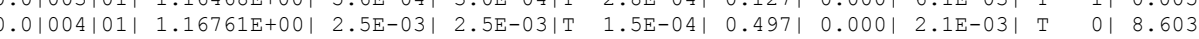

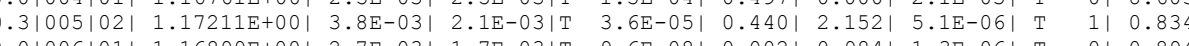

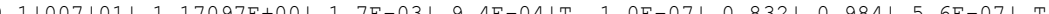
$\begin{array}{lllllll} & \end{array}$

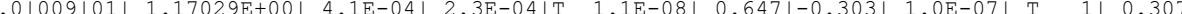
\begin{tabular}{ll|l|l|l|l|l|l|l|l|l|l|l|}
$0.11011|01|$ & $1.17011 \mathrm{E}+00 \mid$ & $4.7 \mathrm{E}-05 \mid$ & $2.9 \mathrm{E}-05 \mid \mathrm{T}$ & $1.7 \mathrm{E}-09 \mid$ & $0.550|-0.303|$ & $2.0 \mathrm{E}-08 \mid \mathrm{T}$ & $1 \mid$ & $0.163 \mid$
\end{tabular}

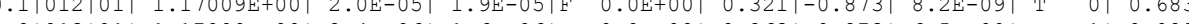

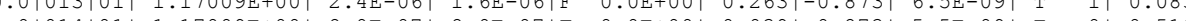

$\begin{array}{llllllllll}0.015|01| & 1.17009 \mathrm{E}+00 \mid & 2.3 \mathrm{E}-12 \mid & 5.8 \mathrm{E}-12 \mid \mathrm{F} & 0.0 \mathrm{E}+00 \mid & 0.273|-1.347| & 4.3 \mathrm{E}-09 \mid & \mathrm{T} & 1 \mid & 0.000 \mid\end{array}$

.LEG-TCHEBY

20

0.007599

0.088013

0.264355

0.050468

0.020508

0.164795

0.1636631
0.164795
.. .6

Within Group Flux

I Preconditioner CG Synth | 208| $101 \mid$ 


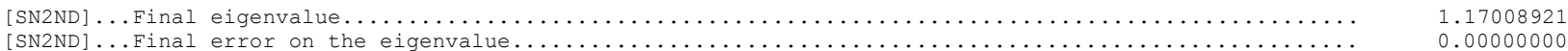

[SN2ND]...Total number of fission source iterations. . .

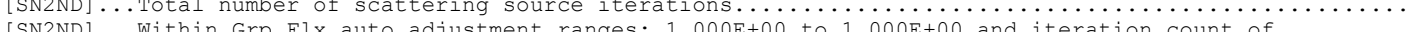

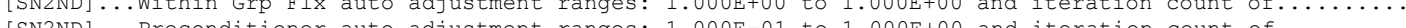

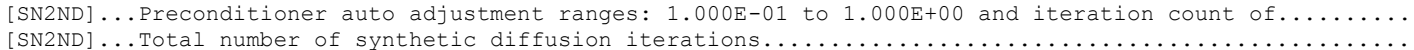

[SN2ND] ... Total computational time required for real solve (seconds) ....

123
111
185

219185

0.894863

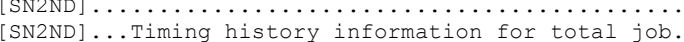

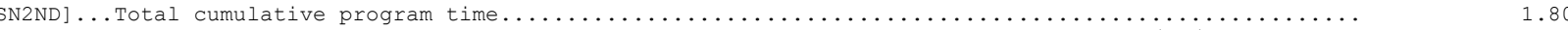

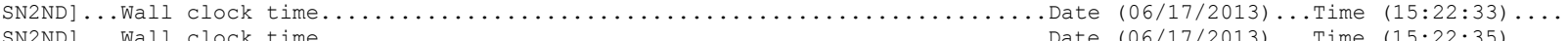

[SN2ND]...Wall clock time. ...

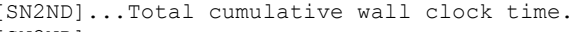

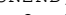

[SN2ND]

SN2ND]

[SN2ND]

[SN2ND]

[SN2ND]

[SN2ND]

[SN2ND]

[SN2ND]

SN2ND

[SN2ND]

[SN2ND]

[SN2ND]

[SN2ND]

$[\mathrm{SN} 2 \mathrm{ND}]$

[SN2ND]

$[\mathrm{SN} 2 \mathrm{ND}]$
$[\mathrm{SN} 2 \mathrm{ND}]$

[SN2ND]

SN2ND

[SN2ND]

[SN2ND]

[SN2ND]

SN2ND]

$[$ [SN2ND]

[SN2ND]

Reading and Trasmitting the Input

$2 \mid$ Group-Angle-Space Segmentation

4/Cross decomposition of the mes

5/Cross Section Communication

6/UNIC native HDF5 export option

7 ISN2ND input checking and PETSC setup

8/SN2ND stenciling of the A matrix

9| SN2ND obtaining the spatial matrices

Rank 0 snapshot of timing events...

Date $(06 / 17 / 2013) \ldots$ Tin

.

1 I NN2ND comptation the boundary condit

2I SN2ND computation of the AC matrices

13|SN2ND flux initialization

14|SN2ND solve of the forward transport equation

15|SN2ND fission source iteration

16/SN2ND scattering source operations

17/SN2ND fission source operations

18|sN2ND within-group flux solution

19

21। SI2ND top levelup odd flux updates

22 | SN2ND $A^{*} x$ operations

23|SN2ND diagonal block CG solve operation (PETSC)

24 | SN2ND P-multigrid prolongation

26। SN2ND P-multigrid restriction

27|SN2ND synthetic diffusion solve (PETSC)

28|SN2ND DSA P-multigrid prolongation

29|SN2ND DSA P-multigrid restriction

301 SN2ND DSA P-multigrid smoothing

31|SN2ND Communicate the angular flux

33|SN2ND SN to PN flux conversion

Paraliel performance timing events.

SN2ND

SN2ND]

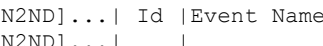

\begin{tabular}{|c|c|c|c|c|}
\hline \multicolumn{2}{|c|}{ Measurements } & \multicolumn{3}{|c|}{ Time (seconds) } \\
\hline gnore 1 & Total I & Avg/Call I & Total | & ercent \\
\hline 0.001 & 31 & 0.00231 & 0.00701 & 0.391 \\
\hline 0.001 & 01 & 0.00001 & 0.00001 & 0.00 \\
\hline 0.001 & 01 & 0.00001 & 0.00001 & 0.00 \\
\hline 0.001 & 01 & 0.00001 & 0.00001 & 0.001 \\
\hline 0.001 & $16 \mid$ & 0.00001 & 0.00001 & \\
\hline 0.001 & 21 & 0.00001 & 0.00001 & 0.001 \\
\hline 0.001 & 11 & 0.00101 & 0.00101 & \\
\hline 0.001 & 11 & 0.00001 & 0.00001 & 0.00 \\
\hline 0.001 & 11 & 0.00001 & 0.00001 & 0.00 \\
\hline 0.001 & 11 & 0.02001 & 0.02001 & \\
\hline .001 & 21 & 0.00601 & 0.01201 & \\
\hline 0.001 & 21 & 0.00101 & 0.00201 & 0.11 \\
\hline 0.001 & 01 & 0.00001 & 0.00001 & 0.00 \\
\hline 0.001 & 21 & 0.88041 & $1.7607 \mid$ & 97.56 \\
\hline 0.001 & 261 & $0.0677 \mid$ & $1.7597 \mid$ & 97.51 \\
\hline 0.001 & $374 \mid$ & 0.00001 & 0.00201 & \\
\hline 0.001 & $213 \mid$ & 0.00001 & 0.00301 & 0.17 \\
\hline 0.001 & $187 \mid$ & $0.0094 \mid$ & $1.7537 \mid$ & 97.171 \\
\hline 0.001 & 4121 & 0.00001 & 0.00101 & \\
\hline 0.001 & 225 & 0.00001 & 0.00001 & 0.001 \\
\hline 0.001 & $225 \mid$ & 0.007 & $1.7067 \mid$ & \\
\hline .001 & $6594 \mid$ & .00011 & 0.4799 & 26.591 \\
\hline 0.001 & 62031 & 0.00021 & 1.09481 & 60.66 \\
\hline .001 & 01 & 0.00 & 0.00001 & \\
\hline 0.001 & 01 & 0.00001 & 0.00001 & 0.001 \\
\hline 0.001 & 01 & 0.00001 & 0.00001 & 0.001 \\
\hline 0.001 & 2251 & 0.00011 & 0.01701 & \\
\hline 0.001 & 01 & 0.00001 & 0.00001 & 0.0 \\
\hline .001 & 01 & 0.000 & 0.00001 & 0.0 \\
\hline 0.001 & 01 & 0.00001 & 0.00001 & 0.00 \\
\hline 0.001 & 63901 & 0.00001 & 0.05101 & 2.83 \\
\hline 0.001 & 6081 & 0.00001 & 0.00201 & \\
\hline .001 & 14321 & 0.00001 & 0.00001 & \\
\hline
\end{tabular}

SN2ND]... $1 \mid$ Reading and Trasmitting the Input

[SN2ND]... 3/METIS decomposition of the mesh

\begin{tabular}{|c|c|c|c|c|}
\hline \multicolumn{5}{|c|}{ Measurements } \\
\hline Max/Min & Avg/Proc & $\operatorname{Max} / \operatorname{Min}$ & | Avg/Proc | & | Percent \\
\hline 1.00 & 31 & 11.9990 & 0.00601 & 0.33 \\
\hline 0.001 & 01 & 0.0000 & 0.00001 & 0.001 \\
\hline 0.001 & 01 & 0.0000 & 0.00001 & 0.00 \\
\hline 0.001 & 01 & 0.0000 & 0.00001 & 0.00 \\
\hline 0.001 & 4 & 0.0000 & 0.00001 & 0.00 \\
\hline 1.001 & 21 & 0.0000 & I $\quad 0.00001$ & \\
\hline \multicolumn{5}{|c|}{ ANL/NE-14/6 (Rev 2.0) } \\
\hline
\end{tabular}


PROTEUS-SN User Manual

July 31, 2015

[SN2ND] $\ldots \mid$ 7|SN2ND input checking and PETSC setup
[SN2ND] ...
8/SN2ND stenciling of the A matrix

[SN2ND] ... 9|SN2ND obtaining the spatial matrices

[SN2ND]... 10|SN2ND treatment of the boundary conditions

SN2ND] ... 11/SN2ND computation of the A matrices

[SN2ND $\ldots . .12$ |SN2ND computation of the PC matrices

[SN2ND] ... 13/SN2ND flux initialization

[SN2ND]... 14/SN2ND solve of the forward transport equation

SN2ND $\ldots . .1$ 15/SN2ND fission source iteration

[SN2ND]... 17/SN2ND fission source operations

[SN2ND] ... 18|SN2ND within-group flux solution

[SN2ND]...| 19/SN2ND within group scattering operations

[SN2ND] $\ldots \mid$ 20|SN2ND within group odd flux updates

SN2ND] ... 21|SN2ND top level CG solve operation

[SN2ND] $\ldots \mid$ 22|SN2ND A* $x$ operations

[SN2ND]... 23/SN2ND diagonal block CG solve operation (PETSC)

[SN2ND] $\ldots \mid$ 24|SN2ND P-multigrid prolongation

[SN2ND]... 25/SN2ND P-multigrid restriction

[SN2ND]... 27|SN2ND synthetic diffusion solve (PETSC)

[SN2ND]... 28/SN2ND DSA P-multigrid prolongation

[SN2ND] $\ldots .291$ SN2ND DSA P-multigrid restriction

[SN2ND] ... 30 ISN2ND DSA P-multigrid smoothing

SN2ND]... 31|SN2ND Communicate the angular flux

SN2ND]... 32|SN2ND global reduce of the PN flux

[SN2ND]... $33 \mid$ SN2ND SN to PN flux conversion

\begin{tabular}{|c|c|c|c|c|}
\hline 1.001 & 11 & 1.00001 & 0.00101 & 0.061 \\
\hline & 11 & 0.00001 & 0.00001 & 0.0 \\
\hline 1.001 & 11 & 0.00001 & 0.00051 & 0.031 \\
\hline .001 & 11 & $1.0501 \mid$ & 0.02021 & 1.121 \\
\hline & 21 & 1.4999 & $0.0095 \mid$ & \\
\hline .001 & 21 & 0.00001 & 0.00071 & \\
\hline .001 & 01 & 0.00001 & 0.00001 & \\
\hline & 21 & $1.0011 \mid$ & 1.76221 & \\
\hline .001 & 261 & 1.0017 & 1.76201 & 97.6 \\
\hline .001 & $374 \mid$ & 1.99991 & 0.00131 & 0.0 \\
\hline .001 & $213 \mid$ & $6.9991 \mid$ & 0.00301 & 0.1 \\
\hline .001 & $187 \mid$ & $1.0029 \mid$ & 1.75621 & 97.31 \\
\hline & 412 & 0.00001 & 0.00031 & \\
\hline .001 & 225 । & 0.00001 & 0.00031 & 0.0 \\
\hline .001 & $225 \mid$ & $1.0118 \mid$ & $1.7027 \mid$ & 94.35 \\
\hline .001 & $6594 \mid$ & 1.07381 & 0.46371 & 25.6 \\
\hline .001 & 62031 & 1.07991 & 1.04611 & 57.96 \\
\hline & 01 & 0.00001 & 0.00001 & \\
\hline & 01 & 0.00001 & 0.00001 & \\
\hline .001 & 01 & 0.0000 & 0.0000 & \\
\hline .001 & 561 & 0.00001 & 0.00421 & \\
\hline .001 & 01 & 0.0000 & 0.0000 & 0.0 \\
\hline .001 & 01 & 0.0000 & 0.0000 & 0.00 \\
\hline & $0 \mid$ & 0.0000 & 0.00001 & \\
\hline & 63901 & 1.7805 & 0.0575 & \\
\hline & 608 & 4.0011 & 0.0057 & 0.3 \\
\hline .001 & 1432 & 0.00001 & $0.0018 \mid$ & 0.1 \\
\hline
\end{tabular}




\section{Appendix G. Finite Elements Used in PROTEUS-SN Along with Vertex and Surface Numbering}

Red indicates elements that are already in PROTEUS.

Blue indicates elements that are to be added to PROTEUS in the future.

Green indicates elements that we may implement into PROTEUS at some point.

Cyan indicates the reference surface numbering for each element.

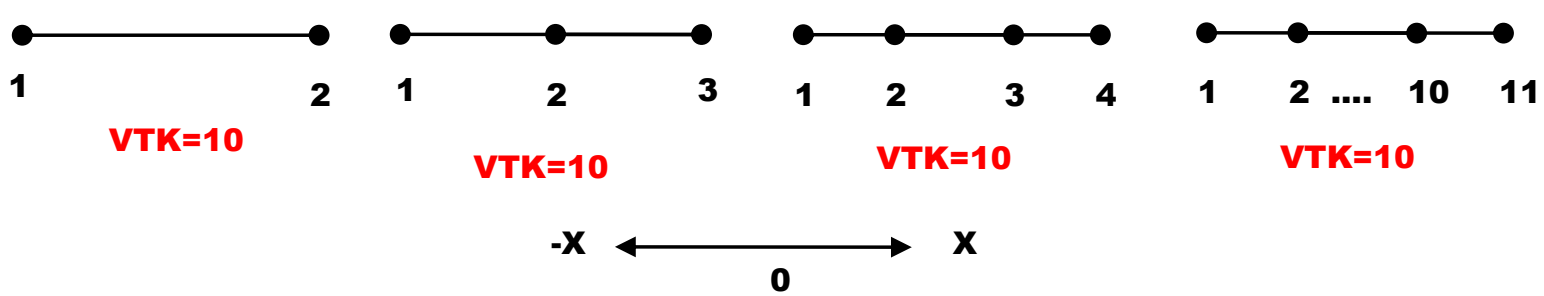

PROTEUS 1-D Finite Element Vertex Layout and VTK Numbering

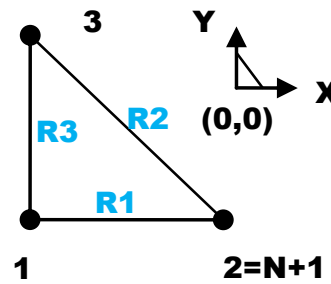

VTK=110

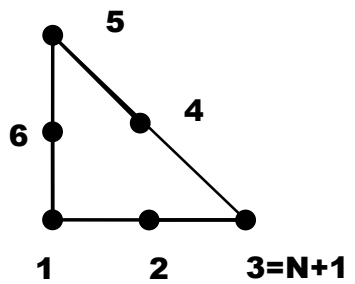

VTK=111

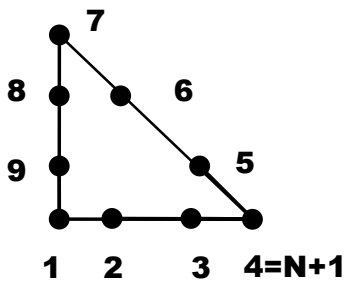

VTK=112

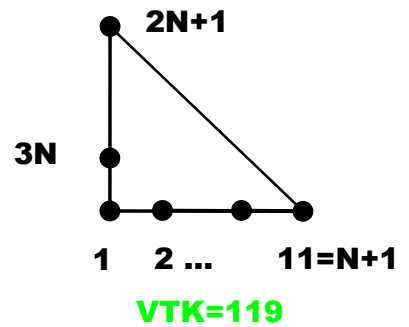

VTK=119

PROTEUS 2-D Triangular Serendipity Finite Element Vertex Layout and VTK Numbering

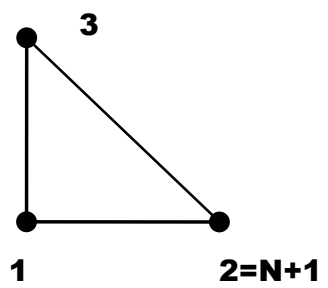

VTK=120

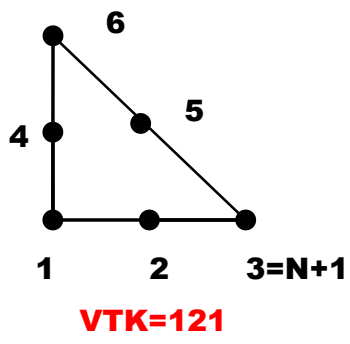

VTK=121

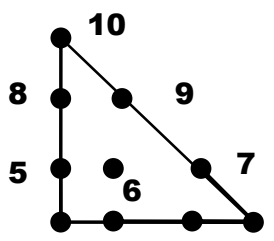

$123 \quad 4=\mathrm{N}+1$

VTK=122

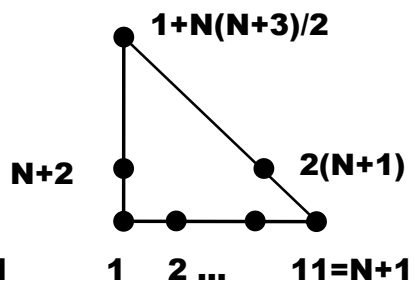

VTK=129

PROTEUS 2-D Triangular LaGrange Finite Element Vertex Layout and VTK Numbering

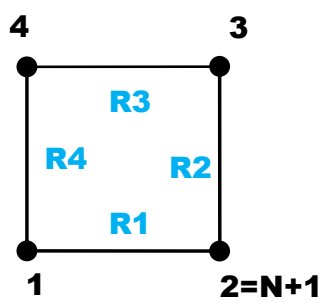

VTK=130
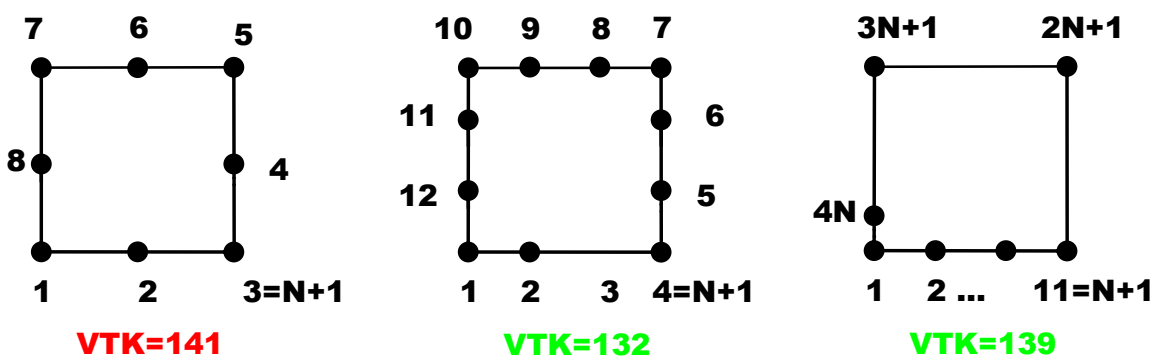

PROTEUS 2-D Quadrilateral Serendipity Finite Element Vertex Layout and VTK Numbering 

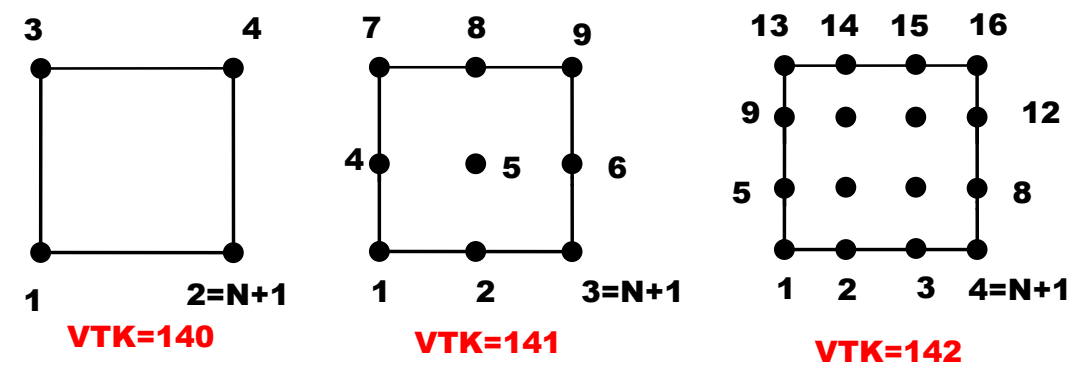

$(\mathbf{N}+1)(\mathbf{N}+1)$

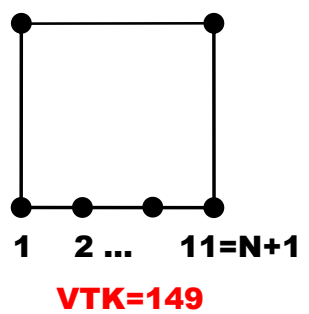

PROTEUS 2-D Quadrilateral LaGrange Finite Element Vertex Layout and VTK Numbering

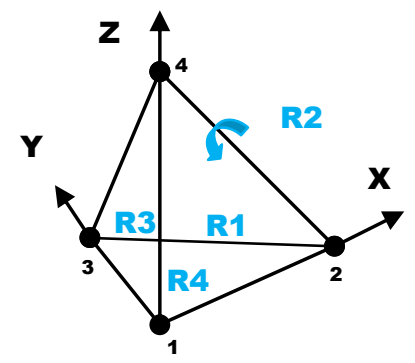

VTK=150

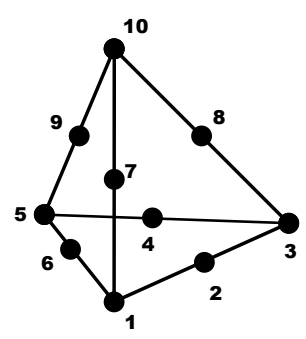

VTK=151

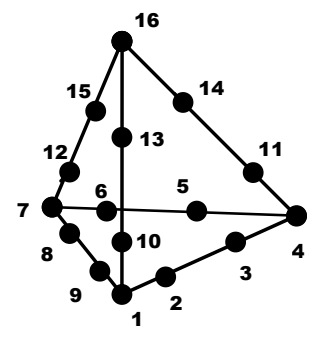

VTK=162

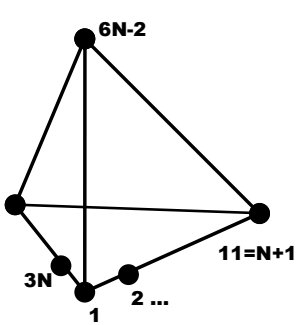

VTK=159

PROTEUS 3-D Tetrahedral Serendipity Finite Element Vertex Layout and VTK Numbering

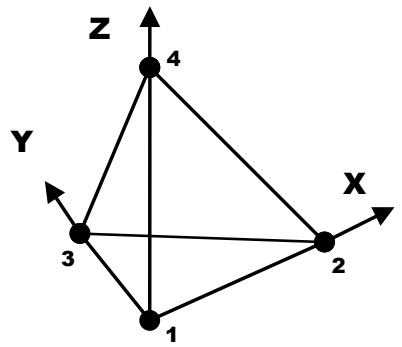

VTK=160

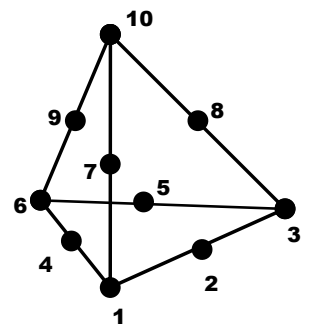

VTK=16

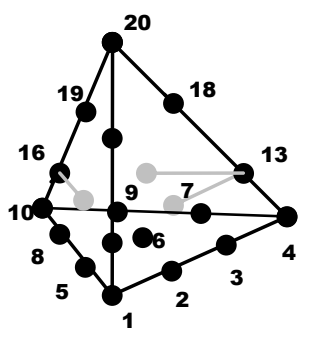

VTK=16

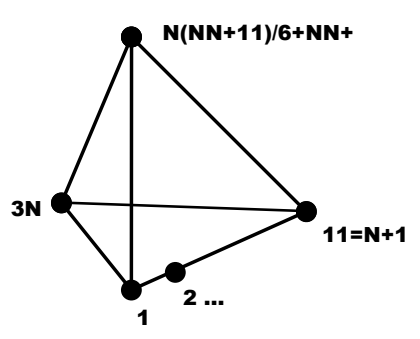

VTK=16

PROTEUS 3-D Tetrahedral LaGrange Finite Element Vertex Layout and VTK Numbering

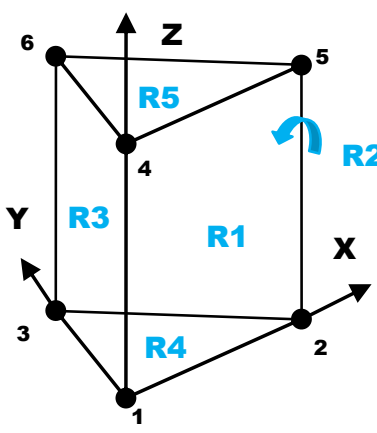

VTK=170

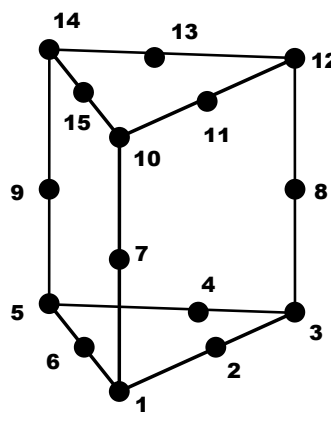

VTK=171

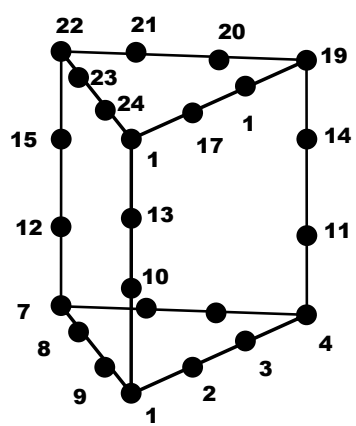

VTK=172

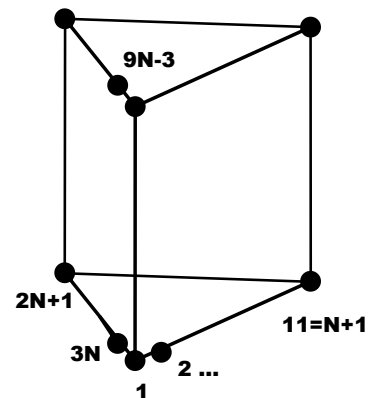

VTK=179

PROTEUS 3-D Wedge Serendipity Finite Element Vertex Layout and VTK Numbering 


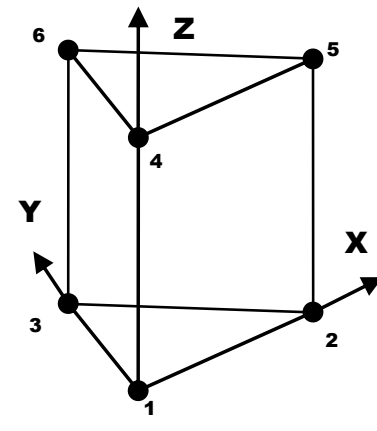

VTK=180

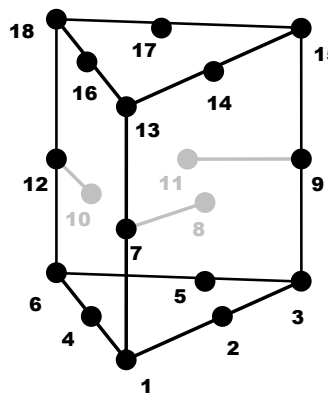

VTK=181

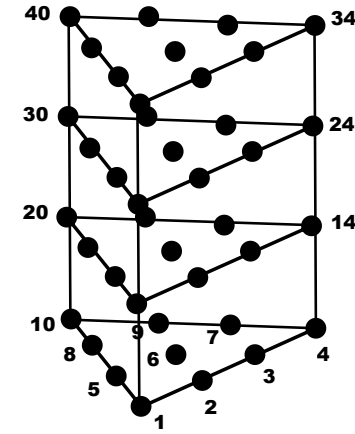

VTK=182

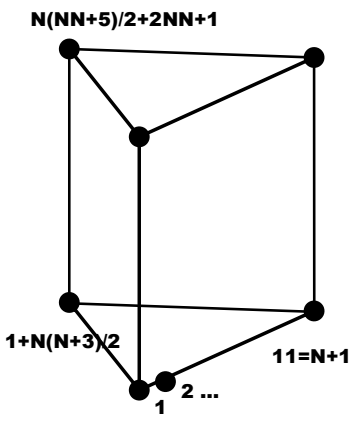

VTK=189

PROTEUS 3-D Wedge LaGrange Finite Element Vertex Layout and VTK Numbering

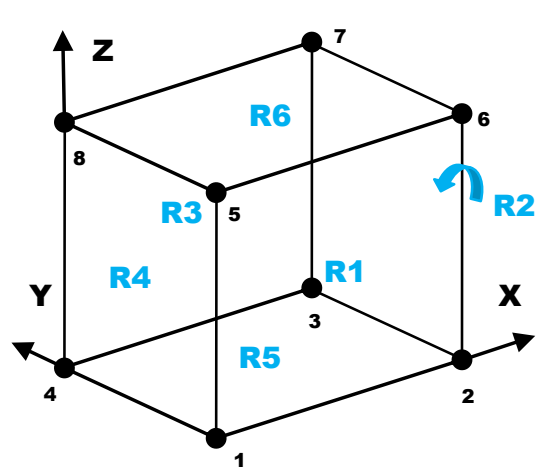

VTK=190

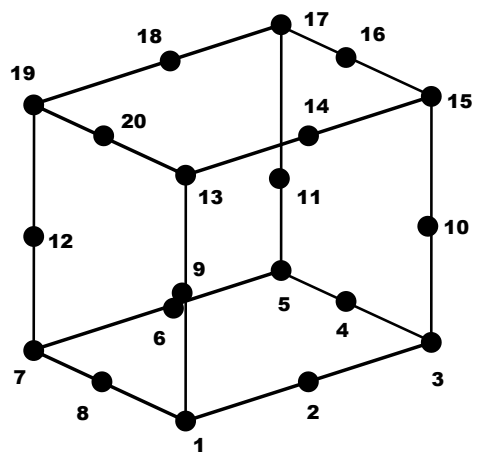

VTK=191

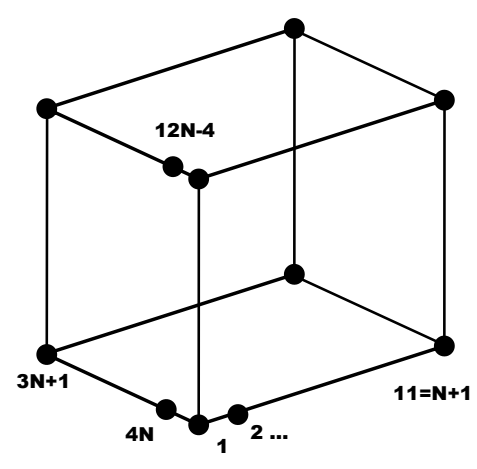

VTK=199

PROTEUS 3-D Hexahedral Serendipity Finite Element Vertex Layout and VTK Numbering

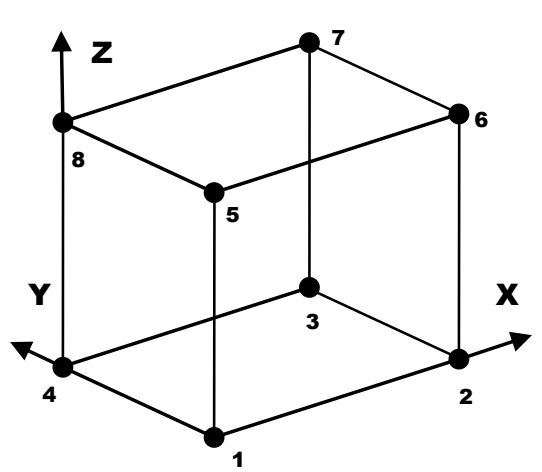

VTK=200

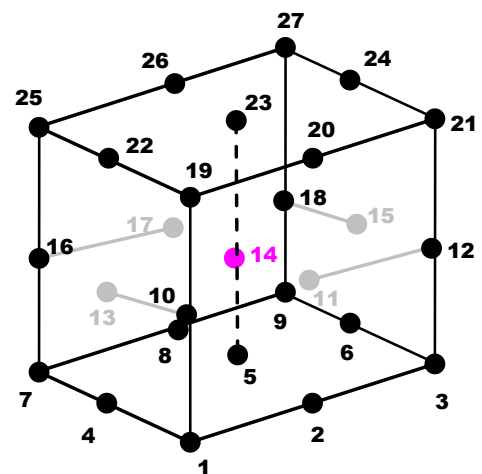

VTK=20

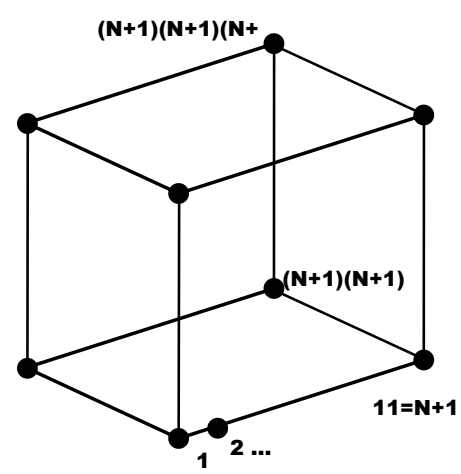

VTK=20

PROTEUS 3-D Hexahedral LaGrange Finite Element Vertex Layout and VTK Numbering 


\section{Argonne}

Nuclear Engineering Division

Argonne National Laboratory

9700 South Cass Avenue, Bldg. 208

Argonne, IL 60439-4842

www.anl.gov 